\title{
Groups with a $p$-element acting with a single non-trivial Jordan block on a simple module in characteristic $p$
}

\author{
David A. Craven \\ Communicated by Christopher W. Parker
}

\begin{abstract}
Let $V$ be a vector space over a field of characteristic $p$. In this paper we complete the classification of all irreducible subgroups $G$ of $\operatorname{GL}(V)$ that contain a $p$-element whose Jordan normal form has exactly one non-trivial block, and possibly multiple trivial blocks. Broadly speaking, such a group acting primitively is a classical group acting on a symmetric power of a natural module, a 7-dimensional orthogonal group acting on the 8-dimensional spin module, a complex reflection group acting on a reflection representation, or one of a small number of other examples, predominantly with a self-centralizing cyclic Sylow $p$-subgroup.
\end{abstract}

\section{Introduction}

The classification of primitive permutation groups that contain a $p^{a}$-cycle (see [32] and [6, p. 229]) has been of great use in answering a variety of problems in permutation group theory. In a different direction, groups generated by transvections have been studied by many authors, culminating in a complete determination in [17]. A simultaneous generalization of these two concepts, when the transvections are unipotent, is the idea of a minimally active element. This is a unipotent element $u$ whose Jordan normal form has at most one block of size greater than 1, and all other blocks of size 1 . (This is equivalent to $\operatorname{dim}\left(C_{M / C_{M}(u)}(u)\right) \leq 1$, or $[M, u] \cap C_{M}(u)$ having dimension at most 1 , where $M$ is the underlying vector space.) Such elements also appeared in work of Oliver, Semeraro and the author [3] in the context of exotic fusion systems. The more general concept of almost cyclic elements in matrix groups has been looked at for sporadic groups [4] and Weil modules for classical groups [5], and minimally active elements for algebraic groups have been studied in [29] and [30]. (An almost cyclic matrix is a matrix that is similar to the sum of a scalar matrix and a matrix whose minimal and characteristic polynomials coincide.)

The author is financially supported by the Royal Society, being awarded a University Research Fellowship. 
In this article we give a general classification theorem for all irreducible subgroups of GL $(M)$ that contain a minimally active element. In some cases, most notably irreducible but imprimitive subgroups, because of the wide range of examples, we give a general construction of such groups but cannot in any real sense give a full classification.

Theorem 1. Let $M$ be a vector space over a field $k$ of characteristic $p$, and let $G \leq \mathrm{GL}(M)$ be an irreducible subgroup. If $G$ contains a unipotent element $u$ such that $\operatorname{dim}\left(C_{M / C_{M}(u)}(u)\right)=1$, then one of the following holds:

(i) $G$ acts imprimitively on $M$, the element $u$ acts on $M$ with a single Jordan block (i.e., $\operatorname{dim}\left(C_{M}(u)\right)=1$ ) and if

$$
M=M_{1} \oplus M_{2} \oplus \cdots \oplus M_{t}
$$

is the finest direct sum decomposition stabilized by $G$, then $t$ is a power of $p$ and $u^{t}$ stabilizes each $M_{i}$ and acts with a single Jordan block on each $M_{i}$.

(ii) $p$ is odd and $M$ factorizes as $M_{1} \otimes M_{2}$ with $\operatorname{dim}\left(M_{i}\right)=2$, and $u$ lies in the central product $\mathrm{SL}_{2}(k) \circ \mathrm{SL}_{2}(k)$ with the obvious action on $M$, with $u$ acting on $M$ with a block of size 3 and a block of size 1 .

(iii) $G$ stabilizes a factorization of $M$ as $M_{1} \otimes M_{2}$ with $\operatorname{dim}(M)=4,8,9$, $p=2,3,2$, respectively, and $o(u)=4,9,8$, respectively, with $u$ acting on $M$ with a single block if $\operatorname{dim}(M)=4,8$, and a block of size 8 and one of size 1 if $\operatorname{dim}(M)=9$.

(iv) $p$ is a Fermat or Mersenne prime $2^{n} \pm 1, \operatorname{dim}(M)=2^{n}, G$ is a subgroup of the extraspecial-type group $\left(Z_{4} \circ 2^{1+2 n}\right) \cdot \mathrm{Sp}_{2 n}(2), o(u)=p$ and $u$ acts with a block of size $p$ and one of size 1 if $p$ is Mersenne, and a single block if $p$ is Fermat,

(v) $p=3, \operatorname{dim}(M)=2^{n}$ for $n=2,3, G$ is a subgroup of the extraspecialtype group $\left(Z_{4} \circ 2^{1+2 n}\right) \cdot \operatorname{Sp}_{2 n}(2), o(u)=3,9$, respectively, and $u$ acts with either a block of size 3 and a block of size 1, or a single block of size 8 .

(vi) the image of $G$ in PGL(M) is almost simple, and $G$ acts absolutely irreducibly.

Cases (i)-(v) are in some sense general, although note that (ii), (iii) and (v) only occur for $\operatorname{dim}(M)<10$, with (i) and (iv) being the generic case. For (i) in particular, and also for the other cases, we give more information and are more specific about which classes occur in the relevant sections. Of course, for (vi) we can be much more specific, and this is the content of the next theorem. We say that $G \leq \mathrm{GL}(M)$ is tensor decomposable if $M=M_{1} \otimes \cdots \otimes M_{t}$ with $G$ stabilizing the factors, so that $G$ is a subgroup of $\operatorname{GL}\left(M_{1}\right) \gtrless \operatorname{Sym}_{t}$. 
Theorem 2. Let $M$ be a vector space over a field of characteristic $p$, and let $G \leq \mathrm{GL}(M)$ be an irreducible subgroup such that the image of $G$ in $\operatorname{PGL}(M)$ is almost simple. Suppose that $G$ acts primitively and tensor indecomposably. If $G$ contains a unipotent element $u$ such that $\operatorname{dim}\left(C_{M / C_{M}(u)}(u)\right)=1$, then (up to automorphism) $G$ is one of the following:

(i) a linear or unitary group acting on a symmetric power of the natural module, a classical group, $G_{2}(q),{ }^{2} G_{2}(q),{ }^{2} B_{2}(q)$, or ${ }^{3} D_{4}(q)$ acting on the natural module (minimal module for $G_{2}$ ), or $\mathrm{PSL}_{3}\left(2^{a}\right) .2$ in characteristic 2 and $M$ of dimension 8 ,

(ii) the group $\operatorname{Spin}_{7}(q)$ acting on the 8-dimensional spin module,

(iii) a subgroup of a complex reflection group acting on a non-trivial composition factor of a reflection representation,

(iv) a group with a self-centralizing cyclic Sylow p-subgroup with $\operatorname{dim}(M) \leq$ $o(u)+1$,

(v) one of the groups

(a) $\mathrm{Alt}_{7} \leq \mathrm{SL}_{4}(2)$,

(b) $2 \cdot \mathrm{Alt}_{7} \leq \mathrm{SU}_{4}(3)$,

(c) $3 \cdot M_{22} \leq \mathrm{SL}_{6}(4)$,

(d) $J_{2} \leq \mathrm{SL}_{6}(4)$,

(e) $3 \cdot M_{10} \leq \mathrm{GL}_{9}(4)$,

(f) $3 \cdot J_{3} \leq \mathrm{SL}_{9}(4)$.

(For (i) of this result, we allow $C_{2}$ to be viewed as $B_{2}$ and $A_{3}$ as $D_{3}$, so they have two "natural" modules.)

We give exact descriptions of all pairs of almost simple groups and simple modules such that the group contains an element acting minimally actively in various results throughout the paper, but there are far too many to list here. Alternating groups are in Propositions 16 and 18, sporadic groups are in Proposition 23, Lie-type groups in characteristic not $p$ are in Propositions 24, 30, 32 and 36, and Lie-type groups in characteristic $p$ are given in Propositions 19, 20 and 21.

Given the results in $[4,5,29,30]$, what remains for almost quasisimple groups is the alternating groups, outer automorphisms of groups of Lie type in defining characteristic, outer automorphisms of sporadic groups, and groups of Lie type in cross characteristic acting on non-Weil modules. After a preliminary section establishing notation and proving some important basic lemmas, in Section 3 we prove Theorem 1. After this, we work with almost simple groups, studying alternating 
groups in Section 4, Lie-type groups in defining characteristic in Section 5 and sporadic groups in Section 6. In Section 7 we give some preliminaries about groups of Lie type in cross characteristic, and then Sections 8 and 9 consider classical and exceptional groups respectively. Finally, Section 10 establishes Theorem 2.

\section{Notation and preliminaries}

Throughout this paper, let $p$ be a prime and let $k$ be an algebraically closed field of characteristic $p$. Let $G$ be a finite group such that $p|| G \mid$, and let $u$ be a $p$-element of $G$.

For specific groups, we write $\mathrm{Alt}_{n}$ for the alternating group of degree $n$, to distinguish it from the algebraic group of type $A$, and write $\mathrm{Sym}_{n}$ for consistency. Similarly, a cyclic group of order $n$ will be denoted $Z_{n}$ rather than $C_{n}$. Groups of Lie type are given their standard names of SL, PSL, PSp, and so on.

All modules considered are finite-dimensional and defined over $k$. We denote the trivial module by $k$ or $k_{G}$ if the group needs to be emphasized, and if $H$ is a subgroup of $G$ and $M$ is a $k G$-module, then $M \downarrow_{H}$ is the restriction of $M$ to $H$. As usual, $\oplus$ and $\otimes$ denote direct sum and tensor product, with $\Lambda^{i}(M)$ and $S^{i}(M)$ denoting the exterior and symmetric powers of $M$.

If $M$ is a $k G$-module and $u$ is a $p$-element, then the action of $u$ on $M$ is conjugate in $\operatorname{GL}(M)$ to a triangular matrix and has a Jordan normal form, made up of blocks of various sizes. If the action of $u$ is conjugate to a triangular matrix with Jordan blocks of sizes $m_{1}, \ldots, m_{r}$, then we say that $u$ has type $\left(m_{1}, \ldots, m_{r}\right)$ on $M$. We often place the $m_{i}$ in weakly decreasing order, but this is not necessary.

The modules of interest are as follows.

Definition 3. Let $G$ be a finite group and let $k$ be an algebraically closed field of characteristic $p>0$. If $u$ is a $p$-element of $G$ and $M$ is a $k G$-module, then $u$ acts minimally actively on $M$ if, in the Jordan normal form of $u$ on $M$, there is at most one Jordan block of size greater than 1, i.e., if $u$ has type $(m, 1, \ldots, 1)$ for some $m \geq 1$ on $M$, or equivalently if $[M, u] \cap C_{M}(u)$ is at most 1-dimensional. We say that $M$ is minimally active if there exists a non-trivial 2-element acting minimally actively on $M$.

Notice that the identity acts minimally actively on all modules, and all $p$-elements act minimally actively on 1-dimensional modules.

We use the term minimally active here, following [3], rather than almost cyclic, following $[4,30]$, because almost cyclic elements need not be $p$-elements, where $p$ is the characteristic of the underlying field, i.e., unipotent elements of the corresponding $\operatorname{GL}(M)$. Since we definitely require this extra hypothesis, we prefer to 
use this more specific term, to avoid leading the reader to believe we have classified all irreducible groups containing almost cyclic elements.

In our work we often need to know how many conjugates of a given element $u$ generate the normal closure of $\langle u\rangle$ in a given group, so we introduce some notation, following [10].

Definition 4. Let $G$ be a finite group and let $u$ be an element of $G$. We denote by $\alpha(u)$ the smallest number of conjugates $u_{1}, \ldots, u_{\alpha(u)}$ of $u$ such that

$$
\left\langle u_{1}, \ldots, u_{\alpha(u)}\right\rangle=\left\langle u^{G}\right\rangle,
$$

i.e., the fewest number of conjugates of $u$ needed to generate the normal closure of $\langle u\rangle$. Write

$$
\alpha(G)=\max _{u \in G} \alpha(u)
$$

Of course, if a group has even order and is not dihedral, then $\alpha(G) \geq 3$, and $\alpha(G)$ is the maximum of $\alpha(u)$ for all elements $u$ of prime order. In [10], various bounds for almost simple groups were obtained, and we will use them frequently to get general constraints on finite groups with elements acting minimally actively on a simple module. For example, in Lemmas 11 and 12 we show that $\alpha(u)=2$ for some specific conjugacy classes of permutations inside symmetric groups.

We collect several basic facts about minimally active modules now.

Lemma 5. Let $G$ be a finite group and let $M$ be a faithful $k G$-module.

(i) If $u$ acts minimally actively on $M$, then $u$ acts minimally actively on any submodule or quotient of $M$, and on the dual of $M$.

(ii) If $u$ is contained in a subgroup $H$ of $G$ and acts minimally actively on $M$, then $u$ acts minimally actively on $M \downarrow_{H}$.

(iii) If $M=M_{1} \oplus M_{2}$ and $u$ acts minimally actively on $M$, then $\left\langle u^{G}\right\rangle$ acts trivially on at least one of the $M_{i}$.

(iv) If $M$ is simple and $u$ acts minimally actively on $M$, then

$$
\operatorname{dim}(M) \leq \alpha(u) \cdot(o(u)-1)
$$

More generally, if $a=\operatorname{dim}(M)-\operatorname{dim}\left(C_{M}(u)\right)$, then $\operatorname{dim}(M) \leq a \cdot \alpha(u)$.

(v) Suppose that $M$ and $N$ are $k G$-modules with $\operatorname{dim}(M) \leq \operatorname{dim}(N)$, and such that $u$ acts non-trivially on $M \otimes N$. We have that $u$ acts minimally actively on $M \otimes N$ if and only if either $\operatorname{dim}(M)=1$ and $u$ acts minimally actively on $N$, or $p$ is odd, $\operatorname{dim}(M)=\operatorname{dim}(N)=2$, and $u$ acts non-trivially (i.e., has type (2)) on both $M$ and $N$. 
(vi) If $u$ acts non-trivially and minimally actively on $\Lambda^{2}(M)$, then $u$ has type (2), $(2,1)$ or (3) on $M$, or $p$ is odd and $u$ has type (4) on $M$.

(vii) If $u$ acts non-trivially and minimally actively on $S^{2}(M)$, then $p$ is odd and $u$ has type (2) on $M$, or $p \geq 5$ and $u$ has type (3) on $M$.

Proof. The first three parts are clear. For the fourth part, note that the codimension $a$ of $C_{M}(u)$ is at most $o(u)-1$, whence the codimension of $C_{M}\left(\left\langle u, u^{g_{2}}, \ldots, u^{g_{r}}\right\rangle\right)$ is at most $r a \leq r(o(u)-1)$. If $G$ is generated by $r$ conjugates of $u$, then this is $C_{M}(G)=0$, so that $\operatorname{dim}(M) \leq r a \leq r(o(u)-1)$, as claimed.

For (v), note that if $\operatorname{dim}(M)=1$ and $u$ acts minimally actively on $N$, then the result is clear, and if $p$ is odd and $u$ acts as a single Jordan block of size 2 on both $M$ and $N$, then $u$ acts on $M \otimes N$ with type $(3,1)$, so one direction holds. For the other, if $\operatorname{dim}(M)=\operatorname{dim}(N)=2$ and $p=2$, then $u$ acts on $M \otimes N$ with type $(2,2)$, and otherwise $\operatorname{dim}(N) \geq 3$. If $u$ acts trivially on $M$, then it must act nontrivially on $N$, and the action of $u$ on $M \otimes N$ contains two copies of the action of $u$ on $N(\operatorname{as} \operatorname{dim}(M) \geq 2)$ so that $u$ cannot act minimally actively. If $u$ acts nontrivially on $M$ and $\operatorname{dim}(N) \geq 3$, then $M$ contains a $u$-invariant subspace on which $u$ acts with type (2), and $N$ contains a $u$-invariant subspace with type either (3) or $(2,1)$. In the first case, $u$ acts on the tensor product of these subspaces as $(4,2)$ (or $(3,3)$ if $p=3)$, and in the second as $(3,2,1)$ (or $(2,2,2)$ if $p=2)$, so $u$ does not act minimally actively in either case, by applying (i).

For the statements about exterior and symmetric powers, recall that

$$
S^{2}(A \oplus B)=S^{2}(A) \oplus(A \otimes B) \oplus S^{2}(B)
$$

and similarly for exterior squares. Thus if $u$ has at least three blocks, then it contains a submatrix of type $(2,1,1)$, and the symmetric and exterior squares of this have two blocks of size 2 . From Table 1 , we see that $u$ acts minimally on $\Lambda^{2}(M)$ and $S^{2}(M)$ when claimed, and that $u$ cannot act minimally on either of these when $(3,1)$ or $(5)$ is a submatrix of the type of $u$ on $M$. All other possibilities are in Table 1, and this completes the proof.

In characteristic 2, we will have to consider modules that are not exterior squares, but exterior squares with one or two trivial composition factors removed.

Lemma 6. Let $p=2$, let $G$ be a finite group and let $M$ be a faithful, simple module of dimension at least 6 . If $V$ is obtained from $\Lambda^{2}(M)$ by removing at most two trivial composition factors, then $V$ is not minimally active for any non-trivial 2-element of $G$.

Proof. The exterior square of a block of size 6 has type $(8,6,1)$, so even a submodule of codimension 2 cannot be minimally active for $u$. Similarly, the exterior 


\begin{tabular}{lll}
\hline Type & Symmetric square & Exterior square \\
\hline$(2)$ & $(3)(p \neq 2)$, & $(1)($ all $p)$ \\
& $(2,1)(p=2)$ & \\
$(2,1)$ & $(3,2,1)(p \neq 2)$, & $(2,1)($ all $p)$ \\
& $(2,2,1,1)(p=2)$ & \\
$(3)$ & $(5,1)(p \neq 2,3)$, & $(3)($ all $p)$ \\
& $(3,3)(p=3)$, & \\
& $(4,2)(p=2)$ & \\
$(3,1)$ & $(5,3,1,1)(p \neq 2,3)$, & $(3,3)($ all $p)$ \\
& $(3,3,3,1)(p=3)$, & \\
& $(4,3,2,1)(p=2)$ & \\
$(4)$ & $(7,3)(p \neq 2,5)$, & $(5,1)(p \neq 2)$, \\
& $(5,5)(p=5)$, & $(4,2)(p=2)$ \\
& $(4,4,2)(p=2)$ & \\
$(5)$ & $(9,5,1)(p \neq 2,5)$, & $(7,3)(p \neq 5)$, \\
& $(5,5,5)(p=5)$, & $(5,5)(p=5)$ \\
& $(8,4,3)(p=2)$ & \\
\hline
\end{tabular}

Table 1. Types of symmetric and exterior squares.

square of a matrix of type $(4,1)$ has type $(4,4,2)$, so again we cannot find a minimally active submodule of codimension 2 for $u$. The exterior square of a matrix of type $(3,1,1)$ has type $(3,3,3,1)$ and that of $\left(2,1^{4}\right)$ has type $\left(2^{4}, 1^{7}\right)$, so again this cannot work. Every type for $u$ acting on $M$ contains one of these types as a submodule, hence $u$ cannot act minimally actively on $V$.

We now give a lemma on when a power of an element can be minimally active. This uses the classification of groups generated by transvections given in [17].

Lemma 7. Let $G$ be a finite group and let $M$ be a faithful, simple $k G$-module. Let $u$ be a p-element and suppose that a non-trivial element $v$ of $\left\langle u^{p}\right\rangle$ acts minimally actively on $M$. Then $v$ acts as a transvection on $M$. Furthermore, $G$ contains a classical group in its natural representation as a normal subgroup, or $p=2, k$ contains $\mathbb{F}_{4}$, and $G$ is either $3 \cdot \operatorname{Alt}_{6} \leq \mathrm{GL}_{3}(k)$ or $3 \cdot \operatorname{PSU}_{4}(3) \leq \mathrm{GL}_{6}(k)$.

Proof. The $p$ th power of a single Jordan block of size $a p$ is the sum of $p$ blocks of size $a$; from this it is easy to see that the $p$ th power of a single block of size $a p+b$ is the sum of $b$ blocks of size $a+1$ and $p-b$ blocks of size $a$. In order 
for this to be minimally active, we must have $a=b=1$. Thus $u$ is the sum of one block of size $p^{a}+1$ for some $a$ and blocks of size at most $p^{a}$, and an element $v$ of order $p$ in $\langle u\rangle$ is a transvection, i.e., has type $\left(2,1^{n-2}\right)$ for $n=\operatorname{dim}(M)$.

Since $G$ possesses a faithful simple module in characteristic $p, O_{p}(G)=1$. (There are many ways to see this: one is that parabolic subgroups act reducibly in the general linear group, and the normalizer of a $p$-subgroup is contained in a parabolic.) The subgroup $H$ generated by all conjugates of $v$ is a normal subgroup of $G$, whence acts semisimply on $M$ as a sum of conjugate modules but also $v$ acts minimally actively, whence $H$ acts irreducibly on $M$ by Lemma 5 (iii). Thus $H$ is an irreducible subgroup of $\operatorname{GL}(M)$, with $O_{p}(H)=1$, containing a transvection, so is one of the groups on Kantor's list in [17, Theorem II].

Of these, we need to check which have a transvection as a proper power of a $p$-element. Classical groups certainly do (cases (T1) and (T2) in Kantor's list), whereas no 2-element powers to a transposition in $\mathrm{Sym}_{n}$ (cases (T3) and (T9)), and case (T6) has Sylow $p$-subgroups of exponent $p$. Cases (T4) and (T8) are not irreducible, and (T5) and (T7) have a single class of involutions, which must be transpositions, and do not have exponent 2 , so are examples. This exhausts the list.

If the Sylow $p$-subgroup of $G$ is cyclic, then we can say more about minimally active modules. This next lemma is a generalization of [3, Propositions 3.7 and 3.9], and the proof follows the same method. We do not give all the background on Green correspondence needed for their proof here, and instead refer to [3, Section 3] and the references therein.

Lemma 8. Let $G$ be a finite group and let $M$ be a faithful, simple $k G$-module. Suppose that the Sylow p-subgroup $U$ of $G$ is cyclic and generated by $u$.

(i) If $N_{G}(U) / U$ is abelian (for example, if $C_{G}(u)=Z(G) \cdot\langle u\rangle$ ), then $u$ acts minimally actively on $M$ if and only if $\operatorname{dim}(M) \leq o(u)+1$.

(ii) If $C_{G}(u)$ is abelian and $M$ is minimally active, then $\operatorname{dim}(M)<2 \cdot o(u)$.

(iii) If $M$ is minimally active, then $\operatorname{dim}(M) \leq o(u)+b$, where $b<\left|C_{G}(u)\right|$.

Proof. By Lemma 7 we may assume that if $M$ is minimally active, that it is $u$ that acts minimally actively.

If $\operatorname{dim}(M)=a \cdot o(u)$ for some integer $a \geq 1$, then $M$ is projective and $u$ acts with $a$ blocks of size $o(u)$; thus $M$ is minimally active if and only if $\operatorname{dim}(M)=$ $o(u)$. Hence we can suppose that $M$ is not projective. Let $V$ denote the Green correspondent of $M$ in $N_{G}(U)$, so that $M \downarrow_{N_{G}(U)}=V \oplus X$, where $V$ is an indecomposable $k N_{G}(U)$-module and $X$ is a relatively $\left\langle u^{p}\right\rangle$-projective $k N_{G}(U)$-module. 
We next aim to understand the action of $u$ on $V$. Note that $V$ is indecomposable and all indecomposable modules for $p$-soluble groups are uniserial, and all composition factors of $V$ lie in the same block of $N_{G}(U)$ and have the same dimension $m$. (This follows since the Brauer tree of a block of a $p$-soluble group is a star.) Thus the action of $u$ is as $m$ blocks of the same size $r$, where $\operatorname{dim}(V)=m r$.

Suppose that $X=0$. Since $u$ acts non-trivially on $M$, this means that $r>1$, so that $m=1$ if and only if $u$ acts minimally actively on $M$. In particular, if $M$ is minimally active, then $\operatorname{dim}(M)<o(u)$. Since $m$ is the dimension of a simple $N_{G}(U) / U$-module, if $N_{G}(U) / U$ is abelian, then this means $m=1$, so if $X=0$, then $u$ acts minimally actively on $M$. This proves all parts of the result when $X=0$.

Since $X \neq 0$, and clearly $u$ acts non-trivially on $X$, it must act trivially on $V$ by Lemma 5 (iii), so $V$ is an indecomposable $k N_{G}(U) / U$-module, hence a simple module as this is a $p^{\prime}$-group. Since $X$ is relatively $\left\langle u^{p}\right\rangle$-projective, by Green's indecomposability criterion we see that all Jordan blocks of $u$ on $X$ have size a multiple of $p$, so there is exactly one, and $\operatorname{dim}(X) \leq o(u)$. This completes the proof of (i) since $\operatorname{dim}(V)=1$ in this case.

Otherwise we need to bound the dimension of a simple $N_{G}(U) / U$-module: since $\left|N_{G}(U) / C_{G}(U)\right|$ has order dividing $p-1$, if $C_{G}(U)$ is abelian, then any simple $N_{G}(U)$-module has dimension at most $p-1$ : thus

$$
\operatorname{dim}(M) \leq o(u)+(p-1)<2 o(u),
$$

as needed. Finally, as $N_{G}(U)$-modules are orbits of $C_{G}(U)$-modules by Clifford's theorem, they have dimension at most $\left|C_{G}(U)\right|-1$ (the ' -1 ' is because the trivial is always in a separate orbit) proving the third part.

We move on to examining $p$-elements acting on direct sum and tensor product decompositions.

Lemma 9. Let $G$ be a finite group and let $u \in G$ be a p-element. Suppose that $u$ acts minimally actively on a faithful, simple $k G$-module $M$. Suppose that $H$ is a normal subgroup of $G$ and that $G=H\langle u\rangle$. Let $1<t=|G: H|$ and suppose that $M \downarrow_{H}$ is the sum of $t$ non-isomorphic simple modules. The action of $u$ on $M$, and of $u^{t}$ on each composition factor of $M \downarrow_{H}$, is as a single Jordan block, of size the dimension of the module.

Conversely, if $u^{t}$ acts on each composition factor of $M \downarrow_{H}$ as a single Jordan block, then $u$ acts minimally actively on $M$ with a single Jordan block.

Proof. We note at the start that $t$ is a power of $p$. Since the restriction of $M$ to $H$ is the sum of $t$ non-isomorphic modules, we have the decomposition

$$
M \downarrow_{H}=M_{1} \oplus M_{2} \oplus \cdots \oplus M_{t},
$$


where $M_{i} \cdot u=M_{i+1}$. Suppose that $m=\left(m_{1}, \ldots, m_{t}\right)$ is a fixed point of $u$, so that in particular each $m_{i} \in M_{i}$ is a fixed point of $u^{t}$. Note that, since $m \cdot u=m$, we must have that $m_{i} \cdot u=m_{i+1}$, whence there is a one-to-one correspondence between the $u$-fixed points of $M$ and the $u^{t}$-fixed points of $M_{1}$, and in particular their dimensions are equal.

Writing $d=\operatorname{dim}\left(M_{1}\right)$, so that $\operatorname{dim}(M)=d t$, if $u$ has type $\left(a, 1^{d t-a}\right)$, then $\operatorname{dim}(M)^{\langle u\rangle}=d t-a+1$. This has to be equal to $\operatorname{dim}\left(M_{1}\right)^{\left\langle u^{t}\right\rangle}$, which is at most $d$. This yields

$$
d t-a+1 \leq d .
$$

First suppose that $a \leq t$, so that $u^{t}=1$. This yields

$$
d t-t+1 \leq d t-a+1=d,
$$

i.e., $(d-1)(t-1) \leq 0$, yielding either $d=1$ or $t=1$, the latter of which is impossible.

Thus suppose that $u^{t} \neq 1$. Since $u$ has type $\left(a, 1^{d t-a}\right)$, we need to know how $u^{t}$ acts: a block of size $a$, when raised to the $t$ th power, has type $\left((\alpha+1)^{\beta}, \alpha^{t-\beta}\right)$, where $a=t \alpha+\beta$ and $0 \leq \beta<t$. Thus the action of $u^{t}$ on $M$ has type

$$
\left((\alpha+1)^{\beta}, \alpha^{t-\beta}, 1^{t d-a}\right) .
$$

These must be distributed equally among the $t$ distinct $M_{i}$, whence $t \mid \beta$ and this means that $\beta=0$. This means that $u^{t}$ acts on $M$ with type $\left(t \alpha, 1^{t(d-\alpha)}\right)$, which means that it acts on each $M_{i}$ with type $\left(\alpha, 1^{d-\alpha}\right)$.

Thus we now have that

$$
1+t(d-\alpha)=\operatorname{dim}(M)^{\langle u\rangle}=\operatorname{dim}\left(M_{1}\right)^{\left\langle u^{t}\right\rangle}=d-\alpha+1,
$$

so $t=1$ (again, impossible) or $d=\alpha$, in other words, $u^{t}$ acts with a single Jordan block, and therefore so does $u$, as claimed.

For the converse, since $u^{t}$ fixes a unique 1-space on each $M_{i}$, any fixed point of $u$ must lie inside this span. But $u^{t}$ acts on this $t$-space as a transitive permutation module, hence fixes a unique 1-space. Thus $u$ acts with a single Jordan block, as claimed.

Along with unipotent elements permuting direct sums, we need unipotent elements permuting tensor products.

Lemma 10. Let $G$ be a finite group and let $u$ be a p-element. Let $H$ be a normal subgroup of $G$ such that $G=H\langle u\rangle$, and let $M$ be a faithful, simple $k G$-module that is not isomorphic to a non-trivial tensor product of two modules, and whose restriction to $H$ factors as a tensor product $M_{1} \otimes M_{2} \otimes \cdots \otimes M_{t}$ of $k H$-modules, where $|G: H|=t>1$ and $\operatorname{dim}\left(M_{i}\right)>1$. If $u$ acts minimally actively on $M$, then 
one of the following holds:

(i) $p=t=2, \operatorname{dim}\left(M_{i}\right)=2,3, u^{2}$ has type $(2,2)$ or $(4,4,1)$ on $M$.

(ii) $p=t=3, \operatorname{dim}\left(M_{i}\right)=2, u^{3}$ has type $(3,3,2)$ on $M$.

Conversely, if $p, t, \operatorname{dim}(M)$ and the action of $u^{t}$ is as above, then $u$ acts minimally actively on $M$.

Proof. First suppose that $u^{t}=1$. Notice that for any element $v_{1}$ in $M_{1}$, writing $v_{i+1}=v_{i} \cdot u$, we can arrange the $M_{i}$ so that $v_{i} \in M_{i}$ and $v_{1} \otimes \cdots \otimes v_{t}$ is fixed by $u$. The subspace spanned by all other monomials in the tensor product is also fixed by $u$, so $M \downarrow\langle u\rangle$ is the sum of a trivial module of $\operatorname{dimension} \operatorname{dim}\left(M_{i}\right)$ and a permutation module with basis the monomials in the tensor product. Since all other orbits than $v_{1} \otimes \cdots \otimes v_{r}$ have length greater than 1 , if $u$ is minimally active, then there is a single orbit on the monomials. However, this is clearly impossible, for example since the number of monomials is $\operatorname{dim}\left(M_{i}\right)^{t}-\operatorname{dim}\left(M_{i}\right)>t$, unless $p=t=\operatorname{dim}\left(M_{i}\right)=2$.

We therefore may assume that $\langle u\rangle \cap H \neq 1$, so that $u^{t}$ is a non-trivial $p$-element. If $u$ acts minimally actively on $M$, then $u^{t}$ has at most $t$ non-trivial blocks. We will prove that, for almost all possible Jordan normal forms of $u^{t}$, there must be more than $t$ non-trivial blocks in its $t$-fold tensor power. Note that, if this is shown for a block of type $\left(\alpha_{1}, \ldots, \alpha_{r}\right)$, then it is shown for any type $\left(\beta_{1}, \ldots, \beta_{s}\right)$ with $s \geq r$ and $\alpha_{i} \leq \beta_{i}$ for all $1 \leq i \leq r$, because for the cyclic group of order $a$, the $k \bar{Z}_{a}$-module with indecomposable summands of dimensions $\alpha_{1}, \ldots, \alpha_{r}$ is a submodule of that with dimensions $\beta_{1}, \ldots, \beta_{s}$, and hence the $t$-fold tensor power of the former is also a submodule of the latter.

Suppose that $p=2$. If $u^{t}$ is a single block of size 2, then the $t$-fold tensor power of the action of $u^{t}$ is as $2^{t-1}$ blocks of size 2 , and this is greater than $t$ for $t \geq 3$. Thus if $t \geq 4$ (as $t$ must be a power of 2), then $u$ cannot act minimally actively at all. Thus we may assume that $t=2$.

If $u^{t}$ is a block of size 3 , then the tensor square has type $(4,4,1)$, so is a candidate. If $u^{t}$ is a block of size 4 , then the tensor square has type $(4,4,4,4)$, so we eliminate all blocks of size at least 4 , leading to the result in the lemma.

We now check that these two cases occur. For $\operatorname{dim}\left(M_{1}\right)=2$, we have that $u$ has order 4 and its square acts as $(2,2)$, so $u$ must have a single block of size 4 . For $\operatorname{dim}\left(M_{1}\right)=3, u$ has order 4 and its square acts as $(4,4,1)$, so $u$ must act as $(8,1)$ (as blocks of sizes 5,6 and 7 square to have types $(3,2),(3,3)$ and $(4,3)$, respectively). Thus $u$ must be minimally active in these cases.

Now suppose that $p$ is odd, and again we consider the $t$-fold tensor power of a block $V$ of size 2 . For the first few tensor powers, we describe them now. In this table we assume that $t<p$. 


\begin{tabular}{ll}
\hline$t$ & type \\
\hline 1 & $(2)$ \\
2 & $(3,1)$ \\
3 & $\left(4,2^{2}\right)$ \\
4 & $\left(5,3^{3}, 1^{2}\right)$ \\
5 & $\left(6,4^{4}, 2^{5}\right)$ \\
6 & $\left(7,5^{5}, 3^{9}, 1^{5}\right)$ \\
\hline
\end{tabular}

These are easily generated as tensoring a block of size $i$ by a block of size 2 yields two blocks, of size $i-1$ and $i+1$, at least when $i<p$. It is easy to see that the start of the $t$-fold product for arbitrary $t$ is

$$
\left(t+1,(t-1)^{t-1},(t-3)^{(t-1)(t-2) / 2-1}, \ldots\right),
$$

and so for $t \geq 5$ there are more than $t$ non-trivial blocks of size less than $t$ in the $(t-1)$-fold tensor power of a single block. This means that there are more than $p$ non-trivial blocks in the $p$-fold power of a single block, and at least two of them have size $p$, for $p \geq 5$. (If $p=3$, tensoring $(3,1)$ by $(2)$ yields $(3,3,2)$, so the second statement holds but not the first.)

For $t>p$, we write this as a single tensor product of $V^{\otimes p}$ and $V^{\otimes(t-p)}$. The first of these contains two blocks of size $p$, whose product with $V^{\otimes(t-p)}$ consists entirely of blocks of size $p$, and hence the product contains at least $2(t-p)>t$ blocks of size $p$ when $t>p$ is a power of an odd $p$.

This proves that $u$ is not minimally active if $p \geq 5$, or $p=3$ and $t \geq 9$. If $p=t=3$ and $V$ is a block of size 3 , then $V^{\otimes 3}$ is the sum of nine blocks of size 3 , and if $V$ is the sum of a 1- and 2-dimensional module, then $V^{\otimes 3}$ has type $\left(3^{5}, 2^{2}, 1^{4}\right)$. Thus if $\operatorname{dim}\left(M_{i}\right) \geq 3$, then we are also done.

We are left with $V$ having dimension 2, in which case $V^{\otimes 3}$ has type $(3,3,2)$, as we saw above. If $u$ has order 9 and $u^{3}$ acts as $(3,3,2)$, then we use the table below that displays the blocks of $u^{3}$, given a block of $u$.

\begin{tabular}{lllllllll}
\hline$u$ & 1 & 2 & 3 & 4 & 5 & 6 & 7 & 8 \\
$u^{3}$ & 1 & $1^{2}$ & $1^{3}$ & $2,1^{2}$ & $2^{2}, 1$ & $2^{3}$ & $3,2^{2}$ & $3^{2}, 2$ \\
\hline
\end{tabular}

We clearly see that $u$ cannot have blocks of size other than 8 , and hence $u$ acts with a single block, as claimed.

The next two lemmas are needed in our analysis of minimally active modules for (central extensions of) symmetric groups. 
Lemma 11. Let $G=\operatorname{Sym}_{n}$ for some $n$, and suppose that $u \in G$ is of order at least 4 and has no cycles of length 1 or 2 . Then $\alpha(u)=2$.

Proof. Of course, $u=(1,2, \ldots, n)$ and $v=(1,2)$ generate $\operatorname{Sym}_{n}$, so $\left\langle u, u^{v}\right\rangle$ has index at most 2 in $\operatorname{Sym}_{n}$, and we see that $\alpha(u)=2$ when $u$ is a single cycle.

Suppose that $u$ has cycle type $\left(m_{1}, \ldots, m_{r}\right)$, with all $m_{i} \geq 3$, and $m_{r} \geq 4$. Write $n_{0}=0, n_{i}=\sum_{j=1}^{i} m_{i}$, and for $1 \leq i \leq r-1$, let

$$
\sigma_{i}=\left(n_{i-1}-(i-2), n_{i-1}-(i-3), \ldots, n_{i}-i, n-i\right),
$$

a cycle of length $m_{i}$. Finally, let

$$
\sigma_{r}=\left(n_{r-1}-(r-2), n_{r-1}-(r-3), \ldots, n-r-1, n, n-r\right),
$$

and let $u$ be the product of the $\sigma_{i}$. The second generator is

$$
v=\left(n_{1}, n-1\right)\left(n_{2}-1, n-2\right) \ldots\left(n_{r-1}-(r-2), n-(r-1)\right)(n-r, n) .
$$

Notice that $u v$ is just the $(n-1)$-cycle $(1, \ldots, n-1)$, and that

$$
\begin{aligned}
{[u, v]=\left(1, n_{1}+1\right) } & \left(n_{1}, n_{2}, n-1\right)\left(n_{2}-1, n_{3}-1, n-2\right) \\
\ldots & \left(n_{r-2}-(r-3), n_{r-1}-(r-3), n-(r-2)\right) \\
& \left(n-r, n-(r-1), n_{r-1}-(r-2), n\right),
\end{aligned}
$$

so that $[u, v]^{6}$ is a double transposition. Letting $H=\langle u, v\rangle$, we note that $H$ is transitive and contains an $(n-1)$-cycle, hence 2-transitive and so primitive. Since it contains a double transposition, and by [6, Example 3.3.1] a primitive subgroup of $\mathrm{Sym}_{n}$ containing a double transposition contains $\mathrm{Alt}_{n}$ for $n \geq 9$, we get $\alpha(u)=2$ in this case as well.

The remaining cases to check are for $n=7,8$ and $u$ with cycle type $(4,3)$, $(5,3)$ and $(4,4)$. In the first case, $\operatorname{Sym}_{7}$ is generated by $(1,2,3,4)(5,6,7)$ and $(1,2,3,5)(4,6,7)$, and in the second and third cases, the group Alt 8 is generated by $(1,2,3,4,5)(6,7,8)$ and $(1,2,3,4,6)(5,7,8)$, and also by $(1,2,3,4)(5,6,7,8)$ and $(1,2,5,6)(4,3,7,8)$.

Lemma 12. Let $G=\operatorname{Sym}_{n}$ for some $n \geq 9$. If $u \in G$ has cycle type $(n-2,2)$, $(n-4,2,2)$ or $(n-6,2,2,2)$, then $\alpha(u)=2$. If $n=10$ and $u$ has cycle type $(4,4,2)$, then $\alpha(u)=2$ also.

Proof. In the first case, let

$$
u=(1, n-1)(2,3, \ldots, n-3, n, n-2), \quad v=(2, n-1)(n-2, n) .
$$

Again, $u v=(1,2, \ldots, n-1)$ and the same proof applies as Lemma 11, as $v$ is itself a double transposition. 
In the second case, let

$$
\begin{aligned}
& u=(1, n-1)(2, n-2)(3, \ldots, n-4, n, n-3), \\
& v=(2, n-1)(3, n-2)(n-3, n) .
\end{aligned}
$$

Then $u v$ is as in the previous case, but now we need to find an element of small support, and this is

$$
[u, v]=(1,3, n, n-3, n-2)(2,4, n-1),
$$

and so $[u, v]^{5}$ is a 3 -cycle, and we are done.

In the third case, let

$$
\begin{aligned}
& u=(1, n-1)(2, n-2)(3, n-3)(4, \ldots, n-5, n, n-4), \\
& v=(2, n-1)(3, n-2)(4, n-3)(n-4, n) .
\end{aligned}
$$

Again, $u v$ is as before, but if $n \geq 10$, then

$$
[u, v]=(1,3,5, n-2)(2,4, n, n-4, n-3, n-1),
$$

and $[u, v]^{6}=(1,5)(3, n-2)$, as needed. If $n=9$, then $u^{2}$ is a 3 -cycle, and we are again done.

Finally, we simply give generators of $\mathrm{Sym}_{10}$ of the appropriate cycle types:

$$
(1,2,3,4)(5,6,7,8)(9,10) \text { and }(1,5,4,7)(2,3,8,10)(6,9) \text {. }
$$

This completes the proof.

We also need to determine better bounds on $\alpha(u)$ for $u$ a unipotent element in $\mathrm{GL}_{n}(2)$ than $\alpha(u) \leq n$ given in [10]. While this bound is sharp for transvections, simply by considering the fixed-point subspace, we need elements close to regular elements. Indeed, by [8], with the exception of $\mathrm{SL}_{4}(2), \mathrm{SL}_{n}(p)$ is generated by two regular unipotent elements for all primes $p$, and all $n \geq 3$. We give this in a lemma for reference.

Lemma 13 ([8]). Let $G=\mathrm{SL}_{n}(p)$ for some $n \geq 2$. If $u$ is a regular unipotent element of $G$, then $\alpha(u)=2$.

Proof. This is proved in [8] for all cases except for $\mathrm{SL}_{4}(2)=\mathrm{Alt}_{8}$, where the regular unipotent class is in bijection with the class containing $u=(1,2,3,4)(5,6)$. Letting $v=(1,5,7,8)(4,6)$, we note that $\langle u, v\rangle$ generates a primitive subgroup of Alt $_{n}$ containing $\left(u v^{2}\right)^{5}=(5,8,6)$. This completes the proof.

From this, we can get that if $u$ is a 2-element of maximal order in $\mathrm{SL}_{n}(2)$, then $\alpha(u) \leq 4$; it is likely that this could be improved still further, but not without considerably more work. 
Lemma 14. Let $G=\mathrm{SL}_{n}(2)$, and let $u$ be a unipotent element of maximal order in $G$. If $n$ is even, then $\alpha(u) \leq 3$ and if $n$ is odd then $\alpha(u) \leq 4$.

Proof. Write $V$ for the natural module for $G$. Suppose that $u$ has type $\left(a, 1^{n-a}\right)$ on $V$ for some $a \geq \frac{n}{2}+1$, so that in particular $C_{V}(u)$ has dimension less than $\frac{1}{2} \operatorname{dim}(V)$. Let $u_{1}$ and $u_{2}$ be regular unipotent generators of $\operatorname{SL}_{a}(2)$, written as matrices in $G$, so with type $\left(a, 1^{n-a}\right)$. The action of $H_{1}=\left\langle u_{1}, u_{2}\right\rangle$ on $V$ has a single simple submodule $W$ of dimension $a$, and all other simple submodules trivial.

Write $v_{1}, \ldots, v_{a}$ for a basis of $W$, and extend the basis to $v_{a+1}, \ldots, v_{n}$ on which $H_{1}$ acts trivially. Let $u_{3}$ act as follows:

$$
v_{i} \cdot u_{3}= \begin{cases}v_{1}+v_{n}, & i=1 \\ v_{i}, & 2 \leq i \leq n-a+1 \\ v_{i}+v_{i-1}, & n-a+2 \leq i \leq n\end{cases}
$$

Of course, $u_{3}$ has the correct type. We claim that $H=\left\langle u_{1}, u_{2}, u_{3}\right\rangle$ acts irreducibly on $V$, so let $X$ denote an $H$-submodule of $V$. Since $W$ is a simple $H_{1}$-submodule, either $X \cap W=0$ or $W \leq X$ : if $W \leq X$, then $v_{1} \in X$, so $v_{n} \in X$ and we see that each $v_{i} \in X$, so that $V=X$. Thus $X \cap W=0$, and so $H_{1}$ acts trivially on $X$, yielding $X$ is a subspace of $\left\langle v_{a+1}, \ldots, v_{n}\right\rangle$. However, repeated application of $u_{3}$ to any element of this space eventually leaves it, as we must project onto $v_{a}$, so that $X=0$. Thus $H$ is irreducible on $V$, containing a copy of $\operatorname{SL}_{a}(2)$ acting on $V$ in a non-self-dual way, hence $H \not \leq \mathrm{Sp}(V)$. Since $H$ contains a transvection, we can apply [17, Theorem II]: either $H=G, H$ is a classical group (all contained in $\mathrm{Sp}(V)$ as the characteristic is 2) or a symmetric group (again, contained in $\operatorname{Sp}(V)$ as the simple modules are self-dual), so since $H \not \leq \mathrm{Sp}(V)$, we have that $H=G$, as needed.

If $n-a+1=a$, i.e., $n=2 a+1$, then the above argument fails: in this case, generate $\mathrm{SL}_{n-1}(2)$ with three elements, and use the fourth to get the full $\mathrm{SL}_{n}(2)$.

If the $u_{i}$ have another type, with a single block of size $a$ and various smaller blocks instead, then choose the $u_{i}$ exactly as before: note that every subspace of $V$ stabilized by $u_{i}$ is also stabilized by the previous $u_{i}$, and so since we had an irreducible subgroup before we must have an irreducible subgroup again. Since it still contains a transposition, we still have $\operatorname{SL}_{n}(2)$, as needed.

We end this section by giving the notation used for almost quasisimple groups. Our groups $G$ will have the property that $G=\left\langle F^{*}(G), u\right\rangle$ for some $p$-element $u$, that $G_{0}=F^{*}(G)$ is quasisimple and that $Z(G)=Z\left(G_{0}\right)$. If $M$ is a faithful simple $k G$-module then this yields an embedding of $G$ into $\operatorname{GL}(M)$. Our conditions on $G$ are equivalent to the image $H$ of $G$ in $\operatorname{PGL}(M)$ being almost simple, and $H^{\prime}$ being simple with $H / H^{\prime}$ generated by a $p$-element of $H$. 


\section{Reduction to almost simple groups}

This section uses Aschbacher's classification of maximal subgroups of classical groups [1] (see also [19,25,31], and in particular [19], which modifies the classes of Aschbacher, and whose notation we will use here) to reduce to the case given at the end of the last section, where $G$ is an almost quasisimple group. Thus we have eight classes $\ell_{1}, \ldots, \ell_{8}$ of maximal subgroups, together with almost quasisimple groups $\delta$. We will determine which elements of the $\mathcal{C}_{i}$ contain minimally active elements.

We assume in this section that $G$ is a subgroup of $\operatorname{GL}(M)$ for an $n$-dimensional $k$-vector space $M$, with $u \in G$ being a $p$-element acting minimally actively on $M$. As we are only concerned with irreducible modules, we stipulate that $G$ acts irreducibly on $M$. In particular, $G$ cannot lie in a parabolic subgroup, class $\mathscr{C}_{1}$.

If $G$ acts imprimitively on $M$, then $G$ stabilizes a direct sum decomposition

$$
M=M_{1} \oplus M_{2} \oplus \cdots \oplus M_{t}
$$

of $M$. Taking this decomposition to be as fine as possible, we see that $G$ is a subgroup of $\mathrm{GL}_{n / t}(k) 2 \operatorname{Sym}_{t}$ (Aschbacher's class $\boldsymbol{C}_{2}$ ). The action of $u$ on this group is in Lemma 9: $u$ must act with a single non-trivial block, $t$ is a power of $p$, and $u^{t}$ acts with a single block on each $M_{i}$. Hence $G$ lies in a wreath product $A$ ? $B$, where $u^{t} \in A$ acts on $M_{1}$ with a single Jordan block, and the $t$-cycle $(1, \ldots, t)$ lies in $B$. Furthermore, given such a setup we always obtain an element $u$. Thus there are many groups acting imprimitively on $M$ with minimally active elements.

If we extend the field and the module is no longer irreducible ( $G$ is contained in an extension field subgroup, class $\mathcal{C}_{3}$ ), then we can apply Lemma 5 (iii) to see that $G$ cannot have a minimally active element.

Suppose that $G$ acts primitively and absolutely irreducibly on $M$, and preserves a tensor decomposition

$$
M=M_{1} \otimes M_{2} \otimes \cdots \otimes M_{t},
$$

so that $G$ is a subgroup of $\mathrm{GL}_{m}(k)$ 2 $\operatorname{Sym}_{t}$ with $n=m^{t}$. Now we can apply Lemmas $5(\mathrm{v})$ and 10, which show that one of the following holds:

(i) $n=4$ and $p$ is odd, with $G$ a subgroup of $\mathrm{SL}_{2}(k) \times \mathrm{SL}_{2}(k)$ and $o(u)=p$ acting with type $(3,1)$,

(ii) $n=4$ and $p=2$, with $o(u)=4$ acting with type (4),

(iii) $n=8$ and $p=3$, with $o(u)=9$ acting with type (8),

(iv) $n=9$ and $p=2$, with $o(u)=8$ acting with type $(8,1)$.

We also showed in that lemma that these cases occur, and we will not comment further on this case. These are classes $\mathscr{C}_{4}$ and $\ell_{7}$. 
If $G$ is contained inside a subfield subgroup in the class $\ell_{5}$ (of the form $\left.N_{\mathrm{GL}_{n}(k)}\left(\mathrm{GL}_{n}\left(k_{0}\right)\right)\right)$, then since our property is independent of the field over which we take our module $M$, we replace $k$ by $k_{0}$ and so this case can be ignored.

If $G$ is contained inside another classical group (i.e., class $\ell_{8}$ ), then we apply this classification of maximal subgroups to that group instead.

Thus we are left with $\mathcal{C}_{6}$, extraspecial-type subgroups, and $\delta$, which is the focus of all subsequent sections of the paper.

Let $r \neq p$ be a prime, and let $R$ denote an extraspecial group of order $r^{1+2 m}$ for some $m$. If $r=2$, we allow $R$ to be either an extraspecial group or the central product with $Z_{4}$. Note that a faithful, irreducible representation of $R$ in characteristic not $r$ has dimension $n=r^{m}$, so we may embed $R$ into $\mathrm{GL}_{n}(k)$ for $k$ an algebraically closed field of characteristic $p$, and $k$ any field of characteristic $p$ except when $R=Z_{4} \circ r^{1+2 m}$ and $k$ needs a fourth root of unity. Let $G$ denote the normalizer in $\mathrm{GL}_{n}(k)$ of $R$, and let $M$ be the natural module for $G$. More information about $G$ can be found in [19,25,31].

We want to prove that if $G$ contains a minimally active element, then the parameters $r, p, m$ are very tightly controlled. To do so, we need to know something about $G / R$, which is a classical group. In Section 7, particularly Proposition 26, we get information about the orders of $p$-elements of classical groups in characteristic different from $p$. Rather than deferring the proof of this result until then, we include it here, but use the definitions and notation from that section. The reader is recommended to skip the proof of this result until they have reached Section 7; the proof is similar to those contained in Section 8.

Proposition 15. If $u \in G$ acts minimally actively on $M$, then $r=2$ and one of the following holds:

(i) $m$ is an odd prime, $p=2^{m}-1$ is a Mersenne prime, and $u$ has order $p$, acting with type $(p, 1)$,

(ii) $m$ is a power of $2, p=2^{m}+1$ is a Fermat prime, and $u$ has order $p$, acting with type $(p-1)$,

(iii) $m=2, p=3, o(u)=3$ acting with type $(3,1)$ (not all elements of order 3 have this property),

(iv) $m=3, p=3, o(u)=9$, acting with type (8).

Proof. If $r$ is odd, then $O^{p^{\prime}}(G)=R \rtimes \mathrm{Sp}_{2 m}(r)$ is a split extension, and so if $u$ is a $p$-element of $G$, we may assume that $u$ lies in $H=\operatorname{Sp}_{2 m}(r)$. By [9, Section 5], if $p$ is odd, then $H$ acts on $M$ as the direct sum of the two Weil modules, of dimensions $\frac{1}{2}\left(r^{m}-1\right)$ and $\frac{1}{2}\left(r^{m}+1\right)$, and so $u$ cannot act minimally actively on $M$ by Lemma 5 (iii). If $p=2$, then $M$ is uniserial of length 3 (see [9, Lemma 5.2]) 
with socle series $W, k$ and $W$, where $W$ is a Weil module, and since $W$ is not minimally active for $p=2$ by Theorem 28 we eliminate this case as well.

Thus $r=2$ and hence $p$ is odd. Here $G / R \cong H$, where $H=\mathrm{GO}_{2 m}^{ \pm}(2)$ or $\mathrm{Sp}_{2 m}$ (2), and the orthogonal-type groups are contained in $R \cdot \mathrm{Sp}_{2 m}$ (2), so we will work solely with that group. Let $d$ denote the order of 2 modulo $p$, so that $p$ divides $\Phi_{d}(2)$. We have that $\alpha(u) \leq m+3$ in all cases, since $p$ is odd.

Let us suppose that the image in $H$ of $u$ lies in a Levi subgroup of $H$, say $\mathrm{Sp}_{2 m-2 a}(2) \times \mathrm{Sp}_{2 a}(2)$. Taking preimages in $G$ yields a central product $G_{1} \circ G_{2}$, where

$$
\begin{aligned}
& G_{1}=R_{1} \cdot H_{1}=\left(4 \circ 2^{2(n-a)+1}\right) \cdot \operatorname{Sp}_{2(m-a)}(2), \\
& G_{2}=R_{2} \cdot H_{2}=\left(4 \circ 2^{2 a+1}\right) \cdot \operatorname{Sp}_{2 a}(2) .
\end{aligned}
$$

The action of this group on $M$ is a tensor product of actions of the $G_{i}$, and since the tensor product of two modules cannot be minimally active unless they both have dimension at most 2 by Lemma $5(\mathrm{v})$, we have that $m=2$ and $p=3$. This case will be considered later.

Thus we may assume that the Sylow $p$-subgroup of $G$, and hence $H$, does not lie in any proper Levi subgroup of $H$. This in particular means that $d$ divides $2 m$.

Suppose that $d$ is either $2 m$, or $m$ is odd and $d=m$; in both cases $d$ is regular, so $C_{H}(u)$ is abelian and of odd order since the image of $u$ is semisimple in $H$ and therefore $C_{H}(u)$ is reductive. Thus $C_{G}(u)$ splits as the direct product of $C_{R}(u)$ and a subgroup $C$ isomorphic to $C_{H}(u)$. Thus $R=C_{R}(u) \circ[R, u]$, and since $u$ does not lie in a Levi subgroup of $H, C_{R}(u)=Z(R) \leq Z(\operatorname{GL}(M))$. This shows that $C_{G}(u)=Z(R) \times C$, and in particular $C_{G}(u)$ is abelian, so that $2^{m}=\operatorname{dim}(M) \leq 2 o(u)$ if $u$ acts minimally actively on $M$ by Lemma 8 (ii). As $\operatorname{dim}(M)=2^{m}$ and $o(u)\left|\Phi_{d}(2)\right|\left(2^{m} \pm 1\right)$, it follows that if $\left(2^{m} \pm 1\right)$ is not a prime power, then $2 o(u) \leq \frac{2}{3}\left(2^{m} \pm 1\right)<2^{m}$, and so $o(u)=2^{m} \pm 1$. Thus $2^{m} \pm 1=$ $\Phi_{d}(2)$, so that $m$ is either a power of 2 or is a prime, and $2^{m} \pm 1$ is a Fermat or Mersenne prime, or is $9=2^{3}+1$, but 2 has order 2 modulo 3 , not 6 .

In these cases, $C_{H}(u)$ is abelian and indeed is simply $\langle u\rangle$, so the subgroup $C_{G}(u)=Z(R) \times\langle u\rangle$ is cyclic, and we may apply Lemma 8 again to show that $u$ acts minimally actively on $M$, and we are done.

If $d=m$ for $m$ even, then the Sylow $p$-subgroup of $H$ has rank 2, and lies inside the Levi subgroup $\mathrm{Sp}_{m}(2) \times \mathrm{Sp}_{m}$ (2), so $u$ can only act minimally actively if $m=1$, i.e., $p=3$, as we saw above.

Suppose that the Sylow $p$-subgroup of $G$ is abelian, so that $o(u)$ is a divisor of $\Phi_{d}(2)$, for $d \mid 2 m$ with $d \neq m, 2 m$. If $d$ is odd, then $d \leq \frac{m}{2}$, and if $d$ is even then $d \leq \frac{2 m}{3}$ : in the first case, $o(u) \leq\left(2^{m / 2}-1\right)$ and in the second $o(u) \leq\left(2^{m / 3}+1\right)$. Since $\alpha(u) \leq m+3$ and $\operatorname{dim}(M)=2^{m}$, we get

$$
(m+3) \cdot(o(u)-1) \leq 2^{m},
$$


which has solutions only for $m \leq 4$ : noting that $d \neq 1$ and $d \mid 2 m$, we get $d=2$ for $m=3$, 4. In this case $p \mid \Phi_{2}(2)=3$ and the Sylow 3-subgroups of $\operatorname{Sp}_{6}(2)$ and $\mathrm{Sp}_{8}(2)$ are non-abelian, so there are no solutions.

If the Sylow $p$-subgroup of $G$ is non-abelian, then $d$ divides $m$ and also $p d$ is at most $m$ if $d$ is odd and $2 m$ if $d$ is even. Suppose that $p \geq 5$ : then $d \geq 3$, and if $d=3$, then $p=7$, if $d=4$, then $p=5$, and if $d \geq 5$, then $p \geq 11$. If $p=5$, then $m \geq 10$, and if $p \geq 7$, then $m \geq 21$. We have $d \leq \frac{2 m}{5}$, and

$$
o(u) \leq m\left(2^{2 m / 5}-1\right),
$$

where the Weyl contribution is $m$ and the toral contribution is of course $2^{2 m / 5}-1$. Thus the inequality $\alpha(u) \cdot o(u)>\operatorname{dim}(M)$ for $u$ to act minimally actively becomes

$$
m(m+3)\left(2^{2 m / 5}-1\right) \geq 2^{m}
$$

for $m \geq 10$, and the only solutions are for $m=10,11,12$, so we must have $p=5$. In this case $o(u) \leq 25$ so we get $25(m+3) \leq 2^{m}$ for $m \geq 10$, and this obviously has no solutions.

We therefore have $p=3$ so $d=2$, and $o(u) \leq 3 m$. This yields the inequality

$$
(3 m-1)(m+3) \geq 2^{m},
$$

which is satisfied for $m \leq 7$. The Sylow 3-subgroup of $\operatorname{Sp}_{2 m}$ (2) lies inside the Levi subgroup $\mathrm{Sp}_{6}(2) \times \mathrm{Sp}_{2 m-6}$ (2) for $4 \leq m \leq 7$, so these cases need not be considered.

We now collect together the cases we need to check, which are only $m=2,3$ for $p=3$. For $\left(Z_{4} \circ 2^{1+4}\right) \cdot \mathrm{Sp}_{4}(2)$, we have that the element $u$ is contained in $\left(Z_{4} \circ 2^{1+4}\right) .\left(\operatorname{Sp}_{2}(2) \times \mathrm{Sp}_{2}(2)\right)$, so the action of $u$ on $M$ can be factored as the tensor product of two matrices. If $u$ lies in one of the factors, then this would have type $\left(2^{2}\right)$, but if it is diagonal, then it would act as $2 \otimes 2$, so type $(3,1)$, minimally active.

For $\left(Z_{4} \circ 2^{1+6}\right) \cdot \operatorname{Sp}_{6}(2) \leq \operatorname{SL}_{8}(9)$, if $o(u)=3$, then $\alpha(u) \leq 4$ (and hence $u$ cannot act minimally actively) unless $u$ lies inside a Levi subgroup $\mathrm{Sp}_{2}$, which of course means that $u$ is not minimally active (as $u$ cannot lie inside such a Levi).

Thus $o(u)=9$ : inside $Z_{3} \geq Z_{3} \leq \operatorname{Sym}_{9}$, elements of order 9 square to the fixedpoint-free class of elements of order 3 , and so inside $\operatorname{Sp}_{2}(2)<\operatorname{Sym}_{3} \leq \operatorname{Sp}_{6}(2)$ we see that elements of order 9 power to the class that lies diagonally across all three $\mathrm{Sp}_{2}$ (2) factors. Therefore the action of $u^{3}$ on $M$ has type $(3,3,2)$, as this is the type of the third tensor power of a block of size 2. As blocks of size 5, 6, 7 and 8 power to have types $(2,2,1),(2,2,2),(3,2,2)$ and $(3,3,2)$, respectively, we see that $u$ must have a single block of size 8 , hence is minimally active, as needed.

Thus we have considered all of the $\mathcal{C}_{i}$, and so we may assume that $G$ is a member of $\&$. Furthermore, this completes the proof of Theorem 1. 


\section{Alternating groups}

In this section we use the notation at the very end of Section 2: let $G_{0}$ be a central extension of an alternating group $\operatorname{Alt}_{n}$ for $n \geq 5$, let $u$ be a $p$-element of $G$ such that $G=\left\langle G_{0}, u\right\rangle$. (Note that, since $\operatorname{Out}\left(G_{0}\right)$ is a 2-group, we have that $u \in G_{0}$ unless $p=2$.) When $n=5,6,7,8$ we get very different answers to the general case: for $n=5$ this is because $\mathrm{Alt}_{5}$ is isomorphic to $\mathrm{SL}_{2}(4)$ and $\mathrm{PSL}_{2}(5)$; for $\mathrm{Alt}_{6}$ it is because of the extra outer automorphism, the exceptional triple cover, and the isomorphism with $\mathrm{PSL}_{2}(9)$; for $\mathrm{Alt}_{7}$, it is the exceptional triple cover; and for Alt 8 it is the isomorphism with $\mathrm{SL}_{4}(2)$.

Because of this, the first proposition deals with those four individual groups. Because of their small order, one can check all calculations easily on a computer, and we just say a few words about its proof. In this proposition, cases (ii) and (iii) are written as if they are general statements, which they are, even though there is only one instance of each in the range $5 \leq n \leq 8$.

Proposition 16. Let $G_{0}$ be a central extension of $\operatorname{Alt}_{n}$ for $5 \leq n \leq 8$, and let $u$ be a p-element of $G$ such that $G=\left\langle G_{0}, u\right\rangle$. Let $V$ denote the non-trivial composition factor of the permutation module for $G$. If $u$ acts minimally actively on a nontrivial simple module $M$, then (up to outer automorphism in the case $n=6$ ) one of the following holds:

(i) $G=\mathrm{Alt}_{n}$ for $p$ odd and $G=\mathrm{Sym}_{n}$ for $p=2, u$ is a single cycle of length $p^{a}$ for some $p^{a} \leq n$ and $M=V$,

(ii) $G=\mathrm{Alt}_{n}$ for $n=2^{a}+2, a \geq 2, p=2, u$ has cycle type $\left(2^{a}, 2\right)$ and $M=V$,

(iii) $G=\operatorname{Sym}_{n}$ for $n=2^{a}+4$ with $a \geq 2, p=2$, $u$ has cycle type $\left(2^{a}, 2,2\right)$ and $M=V$, with $u$ acting as $\left(2^{a}, 1,1\right)$,

(iv) $G=\mathrm{Alt}_{5}, p=2, o(u)=2$ and $\operatorname{dim}(M)=2$,

(v) $G=\operatorname{Sym}_{5}, p=2, o(u)=4$ and $\operatorname{dim}(M)=4$,

(vi) $G=$ Alt $_{5}, p=3, o(u)=3$ and $\operatorname{dim}(M)=3$,

(vii) $G=2 \cdot$ Alt $_{5}, p=3, o(u)=3$ and $\operatorname{dim}(M)=2$,

(viii) $G=\mathrm{Alt}_{5}, p=5, o(u)=5$ and $\operatorname{dim}(M)=5$,

(ix) $G=2 \cdot$ Alt $_{5}, p=5, o(u)=5$ and $\operatorname{dim}(M)=2,4$,

(x) $G=\mathrm{PGL}_{2}$ (9) or $G=M_{10}, p=2, o(u)=8$ and $\operatorname{dim}(M)=8$ (three and one representation respectively),

(xi) $G=3 \cdot M_{10}, p=2, o(u)=8$ and $\operatorname{dim}(M)=6,9$, 
(xii) $G=3 \cdot \operatorname{Alt}_{6}, p=2, o(u)=2,4$ and $\operatorname{dim}(M)=3$ (four representations),

(xiii) $G=\operatorname{Alt}_{6}, p=3, o(u)=3$ and $\operatorname{dim}(M)=3$,

(xiv) $G=2 \cdot$ Alt $_{6}, p=3, o(u)=3$ and $\operatorname{dim}(M)=2$ (two representations),

(xv) $G=2 \cdot \operatorname{Alt}_{6}, p=5, o(u)=5$ and $\operatorname{dim}(M)=4$ (two representations),

(xvi) $G=3 \cdot \operatorname{Alt}_{6}, p=5, o(u)=5$ and $\operatorname{dim}(M)=3,6$ (two representations each),

(xvii) $G=6 \cdot \operatorname{Alt}_{6}, p=5, o(u)=5$ and $\operatorname{dim}(M)=6$ (four representations),

(xviii) $G=$ Alt $_{7}, p=2, u=(1,2,3,4)(5,6)$ and $\operatorname{dim}(M)=4$,

(xix) $G=2 \cdot \mathrm{Alt}_{7}, p=3, u$ is the preimage of the element $(1,2,3)(4,5,6)$ and $\operatorname{dim}(M)=4$,

(xx) $G=2 \cdot \operatorname{Alt}_{7}, p=5, o(u)=5$ and $\operatorname{dim}(M)=4$,

(xxi) $G=3 \cdot$ Alt $_{7}, p=5, o(u)=5$ and $\operatorname{dim}(M)=3,6$ (two representations each),

(xxii) $G=6 \cdot$ Alt $_{7}, p=5, o(u)=5$ and $\operatorname{dim}(M)=6$ (four representations),

(xxiii) $G=2 \cdot$ Alt $_{7}, p=7, o(u)=7$ and $\operatorname{dim}(M)=4$,

(xxiv) $G=3 \cdot$ Alt $_{7}, p=7, o(u)=7$ and $\operatorname{dim}(M)=6$ (two representations),

(xxv) $G=6 \cdot$ Alt $_{7}, p=7, o(u)=7$ and $\operatorname{dim}(M)=6$ (four representations),

(xxvi) $G=$ Alt $_{8}, p=2, u=(1,2)(3,4)(5,6)(7,8)$ and $\operatorname{dim}(M)=4$,

(xxvii) $G=$ Alt $_{8}, p=2, u=(1,2,3,4)(5,6,7,8)$ and $\operatorname{dim}(M)=4$, with $u$ acting as $(3,1)$,

(xxviii) $G=$ Alt $_{8}, p=2, u=(1,2,3,4)(5,6)$ and $\operatorname{dim}(M)=4$,

(xxix) $G=2 \cdot$ Alt $_{8}, p=7, o(u)=7$ and $\operatorname{dim}(M)=8$.

In all cases, unless otherwise specified $u$ acts on the simple module $M$ with type $(\operatorname{dim}(M))$ if $\operatorname{dim}(M) \leq o(u)$, and as $\left(o(u), 1^{\operatorname{dim}(M)-o(u)}\right)$ otherwise.

Proof. For $n=5$, when $p$ is odd, we simply check all simple modules for $2 \cdot \mathrm{Alt}_{5}$, and for $p=2$ we check all simple modules for Alt 5 and Sym $_{5}$.

Next, we deal with $n=6$. For $p=5$ we check all simple modules for $6 \cdot$ Alt $_{6}$, and since $N_{G}(u) /\langle u\rangle$ is cyclic, we only need to know which have dimension at most 6 by Lemma 8 , so we get what is above.

For $p=3$ we check all simple modules for $2 \cdot \mathrm{Alt}_{6}=\mathrm{SL}_{2}(9)$, and the answer will be the same as for $\mathrm{SL}_{2}(q)$ in defining characteristic. 
For $p=2$, here we have $\mathrm{Alt}_{6}$, the three extensions of $\mathrm{Alt}_{6}$ by an outer automorphism, so $\mathrm{Sym}_{6}, \mathrm{PSL}_{2}(9)$ and $M_{10}$, the central extension $3 \cdot \mathrm{Alt}_{6}$, and the last group 3. $M_{10}$, since the $M_{10}$ outer automorphism is the only one preserving the centre of $3 \cdot$ Alt $_{6}$. This group is not necessarily well defined, so we give more details now.

Let $G$ be a group of the form $3 \cdot M_{10}$. By a quick computer calculation, $G$ is generated by two conjugates of $u$ for $o(u)=4,8$ and by three if $o(u)=2$, by checking this is true for $M_{10}$.

The only faithful simple modules for $G$ have dimensions 6 and 9, by [16], with the 6 restricting to $3 \cdot$ Alt $_{6}$ as the sum of two non-isomorphic 3-dimensional simple modules. Thus here we are in the situation of Lemma 9, and $u^{2}$ must act with a single Jordan block of these 3s. This means that $o\left(u^{2}\right)=4$ and so $o(u)=8$. Furthermore, the only action of $u$ that squares to Jordan block structure $(3,3)$ is (6), so that $u$ does indeed act minimally actively on $M$ of dimension 6 .

For the module $M$ of dimension 9 , this restricts simply to $3 \cdot \mathrm{Alt}_{6}$, and only $u$ of order 8 could act minimally actively on $M$. This time, we are resigned to constructing the normalizer inside $\mathrm{GL}_{9}(4)$ of $3 \cdot \mathrm{Alt}_{6}$ and simply computing the action of these elements of order 8 , and they do act as $(8,1)$ on the two dual 9-dimensional simple modules.

For $G_{0}$ a central extension of Alt 7 , for $p=5,7, C_{G}(u)=\langle u\rangle Z\left(G_{0}\right)$ and so we only need $\operatorname{dim}(M) \leq p+1$ by Lemma 8 . For $p=3$, we find the two simple modules for $2 \cdot$ Alt $_{7}$ which are minimally active for the conjugacy class of elements of order 3 that have the smallest centralizer. For $p=2$, the outer automorphism inverts the centre of $3 \cdot \mathrm{Alt}_{7}$, so we only need concern ourselves with $G$ one of $\mathrm{Alt}_{7}$, $\mathrm{Sym}_{7}$ and $3 \cdot \mathrm{Alt}_{7}$, all of which are easily constructible.

For Alt ${ }_{8}$, we simply need to consider Alt $t_{8}$ and $2 \cdot$ Alt $_{8}$ for $p$ odd, and Alt $t_{8}$ and $\mathrm{Sym}_{8}$ for $p=2$, which is easy to do directly.

From now on we let $G_{0}$ be a central extension of an alternating group $\mathrm{Alt}_{n}$ for some $n \geq 9$. We first need to decide which elements of $\operatorname{Sym}_{n}$ act minimally actively on the non-trivial composition factor of the permutation module. The next lemma does this.

Lemma 17. Let $G=\mathrm{Sym}_{n}$, and let $V$ denote the non-trivial composition factor of the permutation module for $G$. If $u$ acts minimally actively on $V$, then one of the following holds:

(i) $u$ is a single cycle of length $p^{a}$ for some $p^{a} \leq n$, acts on $V$ with type $\left(p^{a}-2\right)$ or $\left(o(u), 1^{n-o(u)}\right)$,

(ii) $n=2^{a}+2, a \geq 2, p=2, u$ has cycle type $\left(2^{a}, 2\right)$ and acts on $V$ with type $(n-2)=\left(2^{a}\right)$, 
(iii) $n=2^{a}+4$ with $a \geq 2, p=2, u$ has cycle type $\left(2^{a}, 2,2\right)$ and acts on $V$ with type $\left(n-2,1^{2}\right)$,

(iv) $n=3^{a}+3$ with $a \geq 2, p=3$, u has cycle type $\left(3^{a}, 3\right)$ and acts on $V$ with type $(n-1,1)$.

Proof. Let $M$ denote the permutation module for $G$. Note that $V$ is obtained from $M$ by removing either a single trivial summand if $p \nmid n$, or removing a trivial submodule and a trivial quotient if $p \mid n$.

Note that the action of $u$ on $M$ has type the cycle type of $u$, so if $u$ has more than one cycle of length at least 4, more than two cycles of length at least 3 , or more than three non-trivial cycles, then $u$ cannot act minimally actively on $V$, as $V$ is obtained from $M$ by removing at most two trivials.

Therefore we are left with checking the types $\left(m, 1^{n-m}\right),\left(m, 2,1^{n-m-2}\right)$, $\left(m, 2^{2}, 1^{n-m-4}\right)$ and $\left(m, 3,1^{n-m-3}\right)$, with $p$ any prime, 2,2 and 3 , respectively.

First suppose that $u$ fixes a point, so that $u \in \operatorname{Sym}_{n-1}$. The restriction of $V$ to $\operatorname{Sym}_{n-1}$ is either simple if $p \mid n$, or isomorphic to the permutation module on $\operatorname{Sym}_{n-1}$ if $p \nmid n$. From this we can use induction to easily see that if $u$ fixes a point and is minimally active, then $u$ acts like a single cycle, and the type of $u$ on $V$ is as above. Thus it remains to check cycles types of the form $(m, 2),(m, 2,2)$ and $(m, 3)$, for $m=2^{a}, 2^{a}$ and $3^{a}$, respectively.

If $u$ has cycle type $\left(p^{a}, p\right)$ for $p=2,3$, then $u^{p}$ fixes a point, whence its action on $V$ is known from the above working to have $p$ blocks of size $p^{a-1}$ and $p-2$ blocks of size 1 . Since the action of $u$ on the permutation module has Jordan blocks one of size $p^{a}$ and one of size $p$, and the action of $u$ on $V$ is a subquotient of this, it must be that $u$ acts with one block of size $p^{a}$ and $p-2$ of size 1 , as needed.

We now need to consider $u$ of type $\left(2^{a}, 2,2\right)$, which lies inside the subgroup $H=\operatorname{Sym}_{n-4} \times \operatorname{Sym}_{4}$. The permutation module for $H$ is simply the direct sum of the permutation modules for $\mathrm{Sym}_{n-4}$ and $\mathrm{Sym}_{4}$, and has as a subquotient of codimension 4 a semisimple module obtained by removing all four trivials. The action of $u$ on the 2-dimensional simple subquotient of the second summand is trivial, since $(1,2)(3,4)$ lies in the kernel of every simple module for $\operatorname{Sym}_{4}$. Thus $u$ acts on this semisimple module with blocks $(m-2,1,1)$, where $m=2^{a}$, with this semisimple module being itself a subquotient of $V$. As $u^{2}$ acts on $V$ with blocks $\left(\frac{m}{2}, \frac{m}{2}, 1,1,1,1\right)$, we see that the only possibility for the action of $u$ consistent with both piece of information is that it has type $(m, 1,1)$.

Proposition 18. Let $G_{0}$ be a central extension of $\operatorname{Alt}_{n}$ for $n \geq 9$, and let $u$ be a p-element of $G$ with $G=\left\langle G_{0}, u\right\rangle$. Let $V$ denote the non-trivial composition factor of the permutation module for $G$. If $u$ acts minimally actively on a nontrivial simple module $M$, then (up to outer automorphism in the case $n=6$ ) one 
of the following holds:

(i) $G=\mathrm{Alt}_{n}$ for $p$ odd and $G=\mathrm{Sym}_{n}$ for $p=2$, $u$ is a single cycle of length $p^{a}$ for some $p^{a} \leq n$ and $M=V$, and $u$ acts with type $(n)$ or $\left(o(u), 1^{n-o(u)}\right)$,

(ii) $G=\operatorname{Alt}_{n}$ for $n=2^{a}+2, a \geq 2, p=2$, u has cycle type $\left(2^{a}, 2\right)$ and $M=V$, acting with type $\left(2^{a}\right)$,

(iii) $G=\operatorname{Sym}_{n}$ for $n=2^{a}+4$ with $a \geq 2, p=2, u$ has cycle type $\left(2^{a}, 2,2\right)$ and $M=V$, acting with type $(n-2,1,1)$,

(iv) $G=\operatorname{Alt}_{n}$ for $n=3^{a}+3$ with $a \geq 2, p=3$, $u$ has cycle type $\left(3^{a}, 3\right)$ and $M=V$, acting with type $(n-1,1)$,

(v) $G=2 \cdot$ Alt $_{9}, p=3, o(u)=9$ and $\operatorname{dim}(M)=8$, acting with type $(7,1)$,

(vi) $G=2 \cdot$ Alt $_{9}, p=7, o(u)=7$ and $\operatorname{dim}(M)=8$, acting with type $(7,1)$.

Proof. We consider two cases.

Case 1: $\boldsymbol{p}$ odd. We first assume that $G_{0}=\mathrm{Alt}_{n}$. We start by checking that there are no simple modules for $G_{0}$, other than the trivial module and $V$, that have dimension at most $2 n-2$. By [14, Theorem 7 and Table 1] for $n \geq 12$ we have that this holds, and the dimensions of simple modules are known for $n \leq 11$, so we can check that this holds.

We can do the same thing for $G_{0}=2 \cdot$ Alt $_{n}$ : by [20] for $n \geq 12$ we have that all faithful representations of $G_{0}$ are of dimension greater than $2 n-2$. Thus we need to check $9 \leq n \leq 11$ : for all odd primes the minimal degrees are 8,16 and 16, with all other faithful modules have dimension larger than $2 n-2$, unless $n=10$ and $p=5$, in which case the minimal degree is 8 .

For $p=11$ this cannot yield a minimally active module as $C_{G}(u)=\langle u\rangle \cdot Z(G)$ and $\operatorname{so} \operatorname{dim}(M) \leq p+1=12$ by Lemma 8 .

For $p=7$, since $\alpha(u)=2$, we eliminate $n=10,11$, and for $n=9$ we see that $C_{G}(u)=\langle u\rangle \cdot Z(G)$ and so $u$ acts minimally actively on $M$ if and only if $\operatorname{dim}(M) \leq p+1=8$ by Lemma 8 again. Since $\operatorname{dim}(M)=8$, this means they are minimally active.

For $p=5$, we check that $u$ acts on the 8-dimensional simple modules for $2 \cdot$ Alt $_{9}$ as $(4,4)$, so not minimally active, and for $2 \cdot$ Alt $_{10}$ both classes act as $(4,4)$, so again no minimally active faithful modules, hence none for $2 \cdot \operatorname{Alt}_{11}$ either by restriction.

Finally, for $p=3$ we have $o(u)=9$. For Alt9 the 8-dimensional module is minimally active, with action $(7,1)$, but for $n=10,11$ the action on the 16-dimensionals has blocks $(9,7)$, so not minimally active. 
Thus if $u$ is a $p$-element such that $\alpha(u)=2$, the only simple modules on which $u$ acts minimally actively are the trivial and $V$, unless $n=9$ and $G_{0}=2$. Alt . Note that if $o(u)=3$, then since $\alpha(G) \leq \frac{n}{2}$ by [10, Lemma 6.1], we have that $\operatorname{dim}(M) \leq \frac{3 n}{2}$ by Lemma 5 (iv), so $M$ is either trivial or $V$; hence we will assume that $o(u) \geq 5$.

Let $G_{0}=\mathrm{Alt}_{n}$ or $G_{0}=2 \cdot \mathrm{Alt}_{n}$ for some $n \geq 9$. By Lemma 11 , if the image of $u$ in $\mathrm{Alt}_{n}$ has no fixed points, then $\alpha(u)=2$ and we are done by the previous paragraph, so we may assume that the image of $u$ in $\mathrm{Alt}_{n}$ lies in $\mathrm{Alt}_{n-1}$; we restrict a simple module $M$ on which $u$ acts minimally actively to $H=\mathrm{Alt}_{n-1}$ or $H=2 \cdot \mathrm{Alt}_{n-1}$. Suppose that $H=\mathrm{Alt}_{n-1}$ first. If a trivial submodule or quotient lies in this restriction, then $M$ is a composition factor of the permutation module on the cosets of $H$, so is either trivial or $V$. Moreover, since the composition factors of the restriction are minimally active, we know that the composition factors of $M \downarrow_{H}$ are either trivial or copies of $V_{H}$, the corresponding simple module for $H$. Since $u$ acts on $V_{H}$ with a block of size at least $o(u)-2$, if there were more than one composition factor of $M \downarrow_{H}$ isomorphic with $V_{H}$, then these two large Jordan blocks cannot form blocks of the form $o(u), 1^{a}$ unless $2(o(u)-2) \leq o(u)+1$, i.e., $o(u)=5$, and then $\operatorname{dim}\left(V_{H}\right)$ would need a block of size 3 , only possible if $H=\mathrm{Alt}_{5}$, but then $n=6$, which is not allowed. Thus $M \downarrow_{H}$ has at most one copy of $V_{H}$, and can have no trivials as they would have to be submodules or quotients, so $\operatorname{dim}(M) \leq n-1$, as needed.

If $H=2 \cdot \mathrm{Alt}_{n-1}$, then all composition factors of the restriction of $M$ to $H$ are non-trivial. If $n=9$, then from Proposition 16 we see that $p=7$. Since Alt9 is generated by two 7-cycles, $M$ is therefore a module that appears in the computations above. If $n=10$, then again $p=7$, this time by induction and using this proposition. Again, $\alpha(u)=2$, and so $M$ does not exist as it does not appear above. Finally, for $n \geq 11$ there can be no examples as there are no examples for $n=10$. This completes the proof.

Case 2: $\boldsymbol{p}=2$. Here we do not need to consider $2 \cdot \mathrm{Alt}_{n}$ but do need to consider $G=\operatorname{Alt}_{n}$ and $G=\operatorname{Sym}_{n}$. If $\alpha(u)=2$, then again we need that $\operatorname{dim}(M) \leq 2 n-2$, in fact merely $\operatorname{dim}(M) \leq 2(o(u)-1)$. From [14, Theorem 7 and Table 1] we have $\operatorname{dim}(M)>2 n-2$ for $M \neq k, V$ for all $n \geq 15$, and for $n \leq 14$ we have that $o(u) \leq 8$, so we just need $\operatorname{dim}(M) \leq 14$, for which there are two modules for $G=$ Alt9 with this property, both of dimension 8 (but not isomorphic to $V$ ). However, Alt9 does not contain elements of order 8, so these cannot be examples.

If $\alpha(u)=2$, so that $M=V$, then by Lemma 17 we know that $u$ acts minimally actively on $V$ if and only if we are in cases (i)-(iii) of the proposition. Thus we may assume that $\alpha(u)>2$, and in particular we cannot be in the situations given in Lemmas 11 and 12. 
Suppose that $u$ has at least three 2-cycles and does not have order 2: we can write $u=u_{1} u_{2}$, where the supports of the $u_{i}$ are disjoint, and where $u_{1}$ has cycle type $\left(2^{a}, 2,2,2\right)$ for some $a \geq 2$. Write $H_{i}$ for the symmetric group on the support of $u_{i}, H=H_{1} H_{2}=H_{1} \times H_{2}$, and note that the restriction $M \downarrow_{H}$ is minimally active. The simple $k H$-modules are tensor products of simple $k H_{i}$-modules, and by Lemma $5(\mathrm{v})$ a tensor product of two non-trivial simple modules is not minimally active (unless they both have dimension 2), we see that the composition factors of $M \downarrow_{H}$ are (minimally active) simple modules for one of the $H_{i}$. But $H_{1}$ has no non-trivial minimally active modules, so $H_{1}$ lies in the kernel of $M$, clearly nonsense as $M$ is faithful.

Suppose that $u$ has at least one 2-cycle and at least two cycles of length at least 4 . Again, write $u=u_{1} u_{2}$, this time with $u$ the product of all of the cycles of length at least 4 , unless there are exactly two of length exactly 4 , in which case add another 2-cycle. (If $u$ has cycle type $(4,4,2)$, then $\alpha(u)=2$ by Lemma 12.) Defining the $H_{i}$ and $H$ as above, we again note that no non-trivial minimally active modules exist for $H_{1}$, and so get the same contradiction. Since every fixed-point-free element has one of these properties, we have covered all fixed-point-free cases.

Thus $u$ fixes a point and lies inside $H=\operatorname{Sym}_{n-1}$. As for $p$ odd, we restrict to $H$ and note that the exact same proof works, as long as $n \geq 10$. In order to apply the argument for the case $p$ odd to $p=2$, we need to exclude the case $G_{0}=$ Alt $_{9}$, as for Alt $t_{8}$ there are minimally active simple modules other than $k$ and $V$. However, we already checked Alt, so we may do this. Thus $M \downarrow_{H}$ has at most one copy of $V$ and possibly trivial factors, and therefore $M$ is a submodule of the permutation module on $H$, i.e., $M=k$ or $M=V$, as needed.

\section{Lie type in defining characteristic}

In the notation of the end of Section 2 , this section considers $G_{0}$ a central extension of a simple group of Lie type in characteristic $p$.

Let $\boldsymbol{G}$ be a simple, simply connected algebraic group defined over the field $k$, and let $F$ be a Frobenius morphism on $\boldsymbol{G}$. With the exceptions of a few quasisimple groups (e.g., $2 \cdot \Omega_{8}^{+}(2), 3 \cdot \mathrm{PSL}_{2}(9)$, and so on) if $G_{0}$ is a quasisimple group of Lie type, then for some choice of $\boldsymbol{G}$ and $F$ above, $G_{0}=\boldsymbol{G}^{F}$. Moreover, from [7, Table 6.1.3] we see that if $Z\left(G_{0}\right)$ is a $p^{\prime}$-group, which we require in order to have a faithful irreducible module, then $G_{0}$ is always $\boldsymbol{G}^{F}$ (unless $G_{0}$ is an extension of $\mathrm{Sp}_{4}(2)^{\prime}=\mathrm{Alt}_{6}$, which was examined in the previous section). Hence for this section we can always take $G_{0}$ to be the fixed points of $\boldsymbol{G}$ under $F$.

Furthermore, every simple $k G_{0}$-module is the restriction of a simple $k \boldsymbol{G}$-module, and by Steinberg's tensor product theorem, every simple module for $G_{0}$ is 
a tensor product of Frobenius twists of $p$-restricted simple modules. Since tensor products of simple modules cannot be minimally active unless they have dimension 1 or 2 by Lemma 5 (v), we thus consider $p$-restricted simple modules for $\boldsymbol{G}$.

As every unipotent class of $\boldsymbol{G}$ appears in $G_{0}$, when checking the action of a unipotent element on a given $p$-restricted simple module, we can use either $\boldsymbol{G}$ or any of the quasisimple groups $G_{0}$ over the various ground fields. For explicit calculations, we of course will usually choose $G_{0}$ to be the smallest group, so over the field $\mathbb{F}_{p}$.

If $u \in G_{0}$, then the problem has been almost completely solved already, in [29] and [30], which dealt with all types apart from $C$ and $D$ in characteristic 2 . Here we will finish that last case, and also consider the case where $u$ induces an outer automorphism on $G_{0}$. The next proposition completes the proof for $u \in G_{0}$. (In this proposition, (iv) and (v) can be viewed as subcases of (ii), using the isomorphisms $B_{2} \cong C_{2}$ and $A_{3} \cong D_{3}$, but we separate them out for clarity.) We use the notation $L(\lambda)$ for highest weight modules, using the same conventions as in for example [29] and [30] above. For weights of small rank we can write the weight in full, e.g., 110, but for larger rank it is more clear to write as a sum, so the previous example would be $\lambda_{1}+\lambda_{2}$.

Proposition 19. Let $G_{0}$ be a quasisimple group of Lie type, and let $u \in G_{0}$ be a non-trivial unipotent element. If $M$ is a simple $k G_{0}$-module on which u acts minimally actively, then up to outer automorphism of $G_{0}$, one of the following holds:

(i) $G_{0}=\mathrm{SL}_{2}\left(p^{a}\right), M=L(i)$ for $0 \leq i \leq p-1$, or $M=L\left(1+p^{j}\right)$ of dimension 4 for some $1 \leq j \leq \frac{a}{2}$ and $p$ odd, $u$ has type $(i+1)$ and $(3,1)$, respectively,

(ii) $G_{0}$ is of type $A,{ }^{2} A, B$ or $C$ for all primes, $D,{ }^{2} D$ or ${ }^{3} D_{4}$ for $p$ odd ${ }^{2} B_{2}$ for $p=2, M$ is the natural module,

(iii) $p>3, G_{0}=\mathrm{SL}_{3}\left(p^{a}\right)$ or $\mathrm{SU}_{3}\left(p^{a}\right)$, u has type (3) on the natural module, $M=L\left(2 \lambda_{1}\right)$ of dimension $6, u$ has type $(5,1)$,

(iv) $p$ is odd, $G_{0}=\mathrm{SL}_{4}\left(p^{a}\right)$ or $\mathrm{SU}_{4}\left(p^{a}\right)$, u has type (4) or $(2,2)$ on the natural module, $M=L\left(\lambda_{2}\right)$ of dimension 6 , u has type $(5,1)$ or $\left(3,1^{3}\right)$, respectively,

(v) $p$ is odd, $G_{0}=\mathrm{Sp}_{4}\left(p^{a}\right)$, $u$ has type $(4)$ or $(2,2)$ on the natural module, $M=L\left(\lambda_{2}\right)$ of dimension 5 , u has type (5) or $\left(3,1^{2}\right)$,

(vi) $p$ is odd, $G_{0}=\operatorname{Spin}_{7}\left(p^{a}\right)$, $u$ is regular unipotent, $M=L\left(\lambda_{3}\right)$ of dimension $8, u$ has type $(7,1)$,

(vii) $G_{0}=G_{2}\left(p^{a}\right)$ or ${ }^{2} G_{2}\left(3^{2 a+1}\right)$, $u$ is regular unipotent, $M=L\left(\lambda_{1}\right)$ of dimension 7 (or 6 for $p=2$ ), u has type (7) (or (6) for $p=2$ ). 
Proof. If $G_{0}=\mathrm{SL}_{2}\left(p^{a}\right)$, then for $M=L(\lambda) p$-restricted we see that $u$ always acts with a single block, and for $L(\lambda)$ not $p$-restricted we use Lemma $5(\mathrm{v})$, which shows that $p$ is odd and $M$ is the tensor product of two 2-dimensional modules, i.e., $L\left(1+p^{j}\right)$. By applying a field automorphism we may assume that $1 \leq j \leq \frac{a}{2}$.

For $G_{0}$ of type $A$, or types $B, C$ and $D$ and $p$ odd, [29, Theorem 1.3] gives (ii)-(vi). (Note that the case of $p=3$ for $G_{0}=\mathrm{SL}_{3}\left(p^{a}\right)$ was erroneously included in [29], but can be excluded by Lemma 5 (vii).) For $G_{0}$ of exceptional type [30] shows that only (vii) occurs, so we are left with types $B / C$ and $D$ in characteristic 2.

Let $G_{0}=\operatorname{Sp}_{2 n}\left(2^{a}\right)$ first. By [23, Chapter 4] every Jordan block of odd size appears an even number of times, so there can be no element of order 4 in $G_{0}$ that powers to a transvection. Thus if $u$ is not itself a transvection, no power of $u$ is one. By [10], $\alpha(u) \leq n+3$ if $u$ is not a transvection, and of course since $G_{0}$ acts on a $2 n$-dimensional space the order of $u$ is at most $2 a_{n}$, where $a_{n}$ is the smallest power of 2 that is at least $n$. By Lemma 5 (iv) we have

$$
\operatorname{dim}(M) \leq \alpha(u) \cdot(o(u)-1) \leq(n+3)\left(2 a_{n}-1\right) .
$$

(If $u$ is a transvection, then $\alpha(u)=2 n+1$, and so $\operatorname{dim}(M) \leq 2 n+1$, so that $M$ is the natural module.) By [24, Theorems 4.4 and 5.1], if $n \geq 8$, then $M$ is one of the standard module, its symmetric square (does not occur for $p=2$ ), or its exterior square with a trivial removed (with two trivials removed if $n$ is even).

For $n \geq 3$, by Lemma 6 we see that this exterior square cannot be minimally active for $u$, and for $n=2$ the simple modules for $\mathrm{Sp}_{4}(2)$ are the trivial, the natural and its image under the graph automorphism, and the Steinberg. Since $\mathrm{Sp}_{4}(2)=\mathrm{Sym}_{6}$, we use Proposition 16. Therefore we can assume that $M$ is neither the natural $L\left(\lambda_{1}\right)$ nor the non-trivial factor of its exterior square $L\left(\lambda_{2}\right)$, and that $n \geq 3$.

For $3 \leq n \leq 7$, we use [24, Theorem 4.4] to get the following table of lowdimensional modules, with modules listed in order of increasing dimension.

\begin{tabular}{lrl}
\hline$n$ & Bound & Modules \\
\hline 3 & 42 & $100,001,010$ \\
4 & 49 & $1000,0001,0100,0010$ \\
5 & 120 & $10000,00001,01000,00100$ \\
6 & 135 & $\lambda_{1}, \lambda_{2}, \lambda_{6}$ \\
7 & 150 & $\lambda_{1}, \lambda_{2}, \lambda_{7}$ \\
\hline
\end{tabular}


As we have excluded $L\left(\lambda_{1}\right)$ and $L\left(\lambda_{2}\right)$, we need to consider the spin module $L\left(\lambda_{n}\right)$ for $\mathrm{Sp}_{2 n}(2)$ for $3 \leq n \leq 7$, and the modules $L(0010)$ and $L(00100)$ for $\mathrm{Sp}_{8}(2)$ and $\mathrm{Sp}_{10}(2)$, respectively.

For $\operatorname{Sp}_{8}(2), L(0010)$ is 48 -dimensional, and since $\alpha(u) \leq 7$ for $u$ not a transvection, we must have that $o(u)=8$ if $u$ acts minimally actively. If $u$ has type $\left(6,1^{2}\right),(6,2)$ or $(8)$ on the natural module, then an easy computer calculation shows that $u$ has type $\left(8^{4}, 6^{2}, 2^{2}\right),\left(8^{4}, 6^{2}, 2^{2}\right)$ or $\left(8^{6}\right)$, respectively, on $L(0010)$, so cannot be minimally active.

For $\operatorname{Sp}_{10}(2), L(00100)$ has dimension 100 , and since $\alpha(u) \leq 8$ for $u$ not a transvection, we must have that $o(u)=16$ if $u$ acts minimally actively, so $u$ is the regular unipotent element. This element has type $\left(16^{4}, 14,10,6^{2}\right)$ on $L(00100)$, and so is not minimally active.

Finally, let $M=L\left(\lambda_{n}\right)$. Note that the restriction of $M$ to the $\mathrm{Sp}_{2 n-2}$-parabolic has two composition factors, both isomorphic to $L\left(\lambda_{n-1}\right)$. For $n=3$, we compute the Jordan block structure of all unipotent elements on $L\left(\lambda_{3}\right)$ and get the following table.

\begin{tabular}{lll}
\hline Class & Action on $L\left(\lambda_{1}\right)$ & Action on $L\left(\lambda_{3}\right)$ \\
\hline$C_{1}$ & $2,1^{4}$ & $2^{4}$ \\
$A_{1}$ & $2^{2}, 1^{2}$ & $2^{2}, 1^{4}$ \\
$A_{1}^{(2)}$ & $2^{2}, 1^{2}$ & $2^{4}$ \\
$A_{1}+C_{1}$ & $2^{3}$ & $2^{4}$ \\
$C_{2}$ & $4,1^{2}$ & $4^{2}$ \\
$A_{2}$ & $3^{2}$ & $3^{2}, 1^{2}$ \\
$C_{3}\left(a_{1}\right)$ & 4,2 & $4^{2}$ \\
$C_{3}$ & 6 & 6,2 \\
\hline
\end{tabular}

In particular, we see that there are no minimally active elements in $\operatorname{Sp}_{6}(2)$ on $L(001)$. Thus we proceed by induction on $n$. If $u$ in $\mathrm{Sp}_{2 n}(2)$ acts minimally actively on $L\left(\lambda_{n}\right)$, then place $u$ inside an $\mathrm{Sp}_{2 n-2}(2)$-parabolic: it must act minimally actively on the indecomposable module for this group with socle $L\left(\lambda_{n-1}\right)$, whence in particular it acts minimally actively on this submodule by Lemma 5 (i). Since there are no non-trivial elements of $\mathrm{Sp}_{2 n-2}(2)$ that act minimally actively on $L\left(\lambda_{n-1}\right)$ by induction, $u$ must act trivially on $L\left(\lambda_{n-1}\right)$, whence $o(u)=2$. One can see this either because the unipotent radical of the parabolic is elementary abelian, or because the action of $u$ on $L\left(\lambda_{n}\right)$ must have blocks only of size 1 and 2, since there are two composition factors of the restriction of $L\left(\lambda_{n}\right)$ to the parabolic. 
At any rate, this is impossible since $\alpha(u) \leq 2 n+1$ and

$$
\operatorname{dim}(M)=2^{n}>2 n+1 \geq \alpha(u) \cdot(o(u)-1)
$$

by Lemma 5 (iv).

We therefore need to consider groups of type $D$ now. Let $G_{0}=\Omega_{2 n}^{ \pm}(2)$, and note that $\alpha(u) \leq n+3$ by [10, Theorem 4.4] (as transvections induce the graph automorphism on $G_{0}$, so do not lie in $G_{0}$ itself). By placing $G_{0}$ inside $\operatorname{Sp}_{2 n}(2)$, we see that no unipotent element can act minimally actively on $L\left(\lambda_{2}\right)$ since this is the restriction of the corresponding module for $\mathrm{Sp}_{2 n}$ (2). (See for example [26, Table 1, $\left.\mathrm{MR}_{4}\right]$.)

Using the bound $\operatorname{dim}(M) \leq \alpha(u) \cdot(o(u)-1)$ from Lemma 5 (iv), and [24, Theorems 4.4 and 5.1], we get the possible minimally active modules are the natural $L\left(\lambda_{1}\right)$, and $L\left(\lambda_{2}\right)$ (already eliminated above) for $n \geq 9$, and for $4 \leq n \leq 8$ we get the table below.

\begin{tabular}{rrl}
\hline$n$ & Bound & Modules \\
\hline 4 & 49 & $(1000,0010,0001), 0100,(0011,1010,1001)$ \\
5 & 56 & $\lambda_{1}, \lambda_{2},\left(\lambda_{4}, \lambda_{5}\right)$ \\
6 & 135 & $\lambda_{1}, \lambda_{2},\left(\lambda_{5}, \lambda_{6}\right)$ \\
7 & 150 & $\lambda_{1}, \lambda_{2},\left(\lambda_{6}, \lambda_{7}\right)$ \\
8 & 165 & $\lambda_{1}, \lambda_{2},\left(\lambda_{7}, \lambda_{8}\right)$ \\
\hline
\end{tabular}

The brackets indicate the groupings under the outer automorphism group. For $n \geq 5$ we need to check the two half-spin modules, but for $n=4$ there are other modules to check, with the 48-dimensional modules $L(0011)$ and so on only occurring because our bound for $\alpha(u)$ is lax: checking by computer that $\alpha(u)=2$ when $o(u)=8$ inside $\Omega_{8}^{+}(2)$, we can therefore exclude them.

For $n=5$, we note that $D_{5}$ lies inside $E_{6}$ acting as (up to automorphism) $L(0) \oplus L\left(\lambda_{1}\right) \oplus L\left(\lambda_{5}\right)$ on the minimal module for $E_{6}$. As the dimension of $L\left(\lambda_{5}\right)$ is 16 , we therefore need at least $17-o(u)$ trivial Jordan blocks in the action of the corresponding unipotent class of $E_{6}$ on the minimal module: examining [22, Table 5], only the class $A_{1}$ of elements of order 2 have enough blocks of size 1 , and of course if $o(u)=2$, then $\operatorname{dim}(M) \leq 2 n<16$ (using [10, Theorem 4.4] and Lemma 5 (iv)) which does not work either. For $n \geq 6$, we use induction, exactly the same as for type $C$. This completes the proof.

We now consider the case where $u$ induces an outer automorphism on $G_{0}$. We start with $u$ inducing a graph automorphism on an untwisted group $G_{0}$. In this case, $p=2$ unless $G_{0}$ is type $D_{4}$, and then $p=2,3$. We will then examine field 
and mixed field-graph automorphisms on untwisted groups, and finally how the automorphisms of the twisted groups compare with those of the untwisted groups.

In this proposition, (iii) may be thought of as the case $n=3$ of (v), but we separate them for clarity.

Proposition 20. Let $G_{0}$ be a quasisimple group of Lie type in characteristic $p$, and suppose that $u$ lies in the coset of a graph automorphism on $G_{0}$. If $M$ is a minimally active, non-trivial simple module for $G$, then one of the following holds:

(i) $G=\operatorname{SL}_{n}\left(2^{a}\right) .2, M=L\left(\lambda_{1}\right) \oplus L\left(\lambda_{n-1}\right)$ has dimension $2 n$, and $u^{2}$ is the regular unipotent element,

(ii) $G=\mathrm{SL}_{3}\left(2^{a}\right) .2, M=L(11)$ has dimension 8, and u has order 8,

(iii) $G=\mathrm{SL}_{4}\left(2^{a}\right) .2, M=L(010)$ has dimension 6 , and there are four possible classes for $u$,

(iv) $G=\operatorname{Sp}_{4}\left(2^{a}\right) \cdot 2$ for a odd, $M=L(10) \oplus L(01)$ has dimension 8 , and $u^{2}$ is the regular unipotent element,

(v) $G=\mathrm{SO}_{2 n}^{+}\left(2^{a}\right)$ for $n \geq 4$ and $M$ is the natural module $L\left(\lambda_{1}\right)$.

Proof. Since $u$ induces a graph automorphism on $G_{0}$, we have that $G_{0}$ is untwisted by [7, Theorem 2.5.12(f)].

Suppose that $u$ induces a graph automorphism on $G_{0}$ and that $p=2$, so that $G / G_{0}$ has order 2 . We go through each possibility in turn, of type $A$, type $D$, $C_{2}, F_{4}$ and $E_{6}$. (Note that $G_{2}$ only possesses the graph automorphism of order 2 when $p=3$, so this case does not occur.) Notice that, if $M \downarrow_{G_{0}}$ is simple, then by Lemma 10 we may assume that it is 2-restricted or $G_{0}=\operatorname{SL}_{3}\left(2^{a}\right)$ and $M \downarrow_{G_{0}}$ is the product of two 3-dimensional simple modules: but this is never graph stable, so we can ignore this case.

If $G_{0}=E_{6}\left(2^{a}\right)$, then, since $o(u) \leq 32$ and $\alpha(u) \leq 9$, we have the bound $\operatorname{dim}(M) \leq 9 \cdot 31=279$ if $M$ is minimally active, by Lemma 5 (iv). The adjoint module $L\left(\lambda_{2}\right)$ is the only (non-trivial) graph-stable simple module with dimension at most 279, and the action of unipotent elements on this is given in [22]. For $u$ to be minimally active, $u^{2} \in G_{0}$ must have at most two non-trivial Jordan blocks, but this is not the case. If $M \downarrow_{G_{0}}$ is not simple, then by Lemma $9 u^{2}$ acts with a single Jordan block, which is not possible by Proposition 19.

For $G_{0}=F_{4}\left(2^{a}\right)$, the exponent of the Sylow 2-subgroup of $G_{0}$ is 16 , thus $o(u) \leq 32$. Since $\alpha(u) \leq 8$ from [10, Theorem 5.1], this gives $\operatorname{dim}(M) \leq 248$. The dimensions of the simple modules are 1,26, 26, 246, 246, and 676 and above. The (non-trivial) modules of dimension at most 246 are not graph-stable, and so as in the previous case we apply Lemma 9 and Proposition 19 to prove that no examples occur. 
For $G_{0}=\mathrm{Sp}_{4}\left(2^{a}\right)$ (we may assume that $a \geq 2$ since $a=1$ yields $\mathrm{Sym}_{6}$, which has been considered already), there are only four 2-restricted modules: the trivial, the two 4-dimensional modules $L(10)$ and $L(01)$, swapped by the graph automorphism, and $L(11)$ : the regular element acts with four blocks of size 4 on the $L(11)$ so this cannot extend to a minimally active module for $G$, and if $u$ squares to the regular then $L(10) \oplus L(01)$ is minimally active by Lemma 9 , since $u^{2}$ acts with a single Jordan block on $L(10)$. However, $u$ can only induce a graph automorphism of order 2 if the Sylow 2-subgroup of $\operatorname{Out}\left(G_{0}\right)$ has order 2 by [7, Theorem 2.5.12(e)], with the graph automorphism squaring to a field automorphism in the other cases.

For $G_{0}$ of type $D$, note that $G_{0} \cdot 2=\mathrm{SO}_{2 n}^{+}\left(2^{a}\right)$ lies inside $\operatorname{Sp}_{2 n}\left(2^{a}\right)$, with $\operatorname{dim}(M) \leq(n+3) \cdot(o(u)-1)$ unless $u$ is a transvection by [10, Theorem 4.4], and so we get the same bound as for the unipotent elements for the simple group of type $C$ in the proof of Proposition 19. Using the tables from [24], we see that for $n \geq 5$, every simple module for $G$ that satisfies the bound on $\operatorname{dim}(M)$ is the restriction of a simple module for type $C$, and hence is minimally active only if the module is for type $C$. This yields only the natural module, which is of course minimally active. When $n=4$, we get for $G_{0}$ the modules 0000 (trivial), 1000 (natural), $0010 \oplus 0001$ (sum of the two half-spins), 0100 (exterior square of natural), and 0011 of dimension 48 . These are all also the restriction of a module for $\operatorname{Sp}_{8}\left(2^{a}\right)$, and so we are again done.

The last case for $p=2$ is $G_{0}=\mathrm{SL}_{n}\left(2^{a}\right)$. If $M \downarrow_{G_{0}}$ is not simple, then by Lemma 9 we have that $v=u^{2}$ acts as a single Jordan block on each factor: thus $v$ is the regular element and $M$ is the sum of the natural and its dual.

Thus we may assume that $M \downarrow_{G_{0}}$ is simple, i.e., $M \downarrow_{G_{0}}$ is a graph-stable simple module, and 2-restricted by our discussion at the start of the proof. If $v$ has maximal order in $G_{0}$, then $\alpha(u) \leq 4$ by Lemma 14 , and writing $a_{n}$ for the smallest power of 2 that is at least $n$, we have that $\operatorname{dim}(M) \leq 4 \cdot\left(2 a_{n}-1\right)$. We now use [24, Theorems 4.4 and 5.1] to get that $L\left(\lambda_{1}+\lambda_{n-1}\right)$ is the only graph-stable module of dimension at most this for $n \geq 7$. For $\operatorname{SL}_{6}\left(2^{a}\right)$ we have the module $L\left(\lambda_{3}\right)$, with $L(0110)$ for $\mathrm{SL}_{5}\left(2^{a}\right)$. For $n \leq 4$ there are several possibilities and we deal with them later.

If $u$ does not have maximal order, then $o(u) \leq a_{n}$ and $\alpha(u) \leq n$ by [10, Theorem 4.1], so that $\operatorname{dim}(M) \leq n\left(a_{n}-1\right)<2 n^{2}$. We again use [24] to see which graph-stable modules we need to consider: doing so yields smaller bounds than the previous case, and so we need only consider $n=3,4$, and the specific modules for larger groups above.

We quickly show that $L\left(\lambda_{1}+\lambda_{n-1}\right)$ is not minimally active if $n \geq 5$ : it is obtained from the tensor product of the natural and its dual by removing at most two trivial factors, and so if $u$ inducing a graph on $L\left(\lambda_{1}+\lambda_{n-1}\right)$ is minimally 
active, then $v$ acts with at most two non-trivial blocks on the module. As in the proof of Lemma 6, if $v$ acts with at least two non-trivial blocks on $L\left(\lambda_{1}\right)$, then the action of $v$ on the tensor square has at least eight non-trivial blocks, whence $u$ cannot act minimally actively if one removes at most two trivials. If $v$ acts with a trivial block and a block of size at least 3 , then $v$ acts on the tensor square with blocks at least of size $(4,4,3,3,1,1)$, so $u$ cannot be minimally active when removing two trivials. Similarly, if $v$ contains a block of size at least 4 , then $v$ acts on the tensor square with at least four blocks of size at least 4 , and if $v$ acts on the natural with a block of size 2 and at least three trivial blocks, then $v$ acts on the tensor square with at least five blocks of size at least 2, hence again $u$ cannot be minimally active.

The actions of the classes of $\mathrm{SL}_{6}(2)$ on $L\left(\lambda_{3}\right)$ are given in the following table. None of these can be the square of a minimally active element, so we are done.

\begin{tabular}{ll}
\hline Type on $L\left(\lambda_{1}\right)$ & Type on $L\left(\lambda_{3}\right)$ \\
\hline $2,1^{4}$ & $2^{6}, 1^{8}$ \\
$2^{2}, 1^{2}$ & $2^{8}, 1^{4}$ \\
$2^{3}$ & $2^{10}$ \\
$3,1^{3}$ & $3^{6}, 1^{2}$ \\
$3,2,1$ & $4^{2}, 3^{2}, 2^{2}, 1^{2}$ \\
$3^{2}$ & $4^{4}, 1^{4}$ \\
$4,1^{2}$ & $4^{4}, 2^{2}$ \\
4,2 & $4^{4}, 2^{2}$ \\
5,1 & $7^{2}, 3^{2}$ \\
6 & $8^{2}, 2^{2}$ \\
\hline
\end{tabular}

For $\mathrm{SL}_{5}(2)$, the dimension of $L(0110)$ is 74 , so if $o\left(u^{2}\right) \leq 4$, then this module fails the bound $\alpha(u) \cdot(o(u)-1)$, as $\alpha(u) \leq 5$. However, the only element $v$ of $\mathrm{SL}_{5}(2)$ with order 8 is the regular unipotent element, and for this one $\alpha(v)=2$ by Lemma 13, so this module cannot be minimally active.

We are thus left with $n=3,4$. For $n=4$, we examine Proposition 16, which states that the 6-dimensional module $L\left(\lambda_{2}\right)$ is the only graph-stable minimally active module, and here there are several classes that work. For $n=3$, we simply check the simple modules for $\mathrm{PGL}_{2}(7)=\mathrm{SL}_{3}(2) .2$, and an element of order 8 acts with a single Jordan block on both $L(10) \oplus L(01)$, in line with the proposition, and also on the 8-dimensional module $L(11)$, as seen in [5, Theorem 1.2]. 
For $p=3$, we only have the group $\Omega_{8}^{+}\left(3^{a}\right)$ to consider, and since the graph automorphism of order 3 permutes the central involutions of the Spin group regularly, we may assume that $G_{0}$ is simple. If $M$ is minimally active and $M \downarrow_{G_{0}}$ is not simple, then $u^{3}$ must act with a single Jordan block on each composition factor of $M \downarrow_{G_{0}}$ by Lemma 9, but this does not occur by Proposition 19. Thus $M \downarrow_{G_{0}}$ is simple: the smallest non-trivial, graph-stable simple module has dimension 28, and the next smallest has dimension 195 (see [24, Appendix A.41]), and since $\alpha(u)=2$ if $o(u)=9$ and $\alpha(u) \leq 4$ if $o(u)=3$ for $G_{0}=\mathrm{P}_{-}^{+}(3)$, we have that $\operatorname{dim}(M)=28$ and $M=L\left(\lambda_{2}\right)$. Since $u$ is minimally active, $u^{3}$ has at most three non-trivial Jordan blocks. However, of the 27 non-trivial unipotent classes of 3-elements in $\Omega_{8}^{+}$(3), all have at least four non-trivial blocks on $L\left(\lambda_{2}\right)$. Thus there is no candidate for $M$.

Having dealt with the case where $u$ induces a graph automorphism, we are left with the case where $u$ induces either a field automorphism or a mixed field-graph automorphism on the untwisted group $G_{0}$.

Proposition 21. Let $G_{0}$ be a quasisimple group of Lie type in characteristic $p$, and suppose that $u$ induces an outer automorphism on $G_{0}$ that is not a graph automorphism. If $M$ is a minimally active simple module for $G$, then up to outer automorphism of $G$ one of the following holds:

(i) $p=2, G=\mathrm{SL}_{2}\left(2^{2 a}\right) .2, M=L\left(1+2^{a}\right)$ of dimension 4,

(ii) $p=3, G=\mathrm{SL}_{2}\left(3^{3 a}\right) \cdot 3, M=L\left(1+3^{a}+3^{2 a}\right)$ of dimension 8 ,

(iii) $p=2, G=\mathrm{SL}_{3}\left(2^{2 a}\right) \cdot 2$, $u$ induces either a field or the product of a field and graph automorphism on $G_{0}, M=L\left(1+2^{a}, 0\right)$ or $M=\left(1,2^{a}\right)$, respectively, both of dimension 9 ,

(iv) $p=2, G=\mathrm{SU}_{3}\left(2^{2 a}\right) .2$, $u$ induces the unique outer automorphism of order 2 on $G_{0}, \operatorname{dim}(M)=9$ is such that $M \downarrow_{G_{0}}$ is simple,

(v) $G=G_{0} . t, M \downarrow_{G_{0}}=M_{1} \oplus \cdots \oplus M_{t}$ with $u^{t} \in G_{0}$ acting on each $M_{i}$ with a single Jordan block, the possibilities for which are given in Proposition 19.

Proof. We begin with the case where $G_{0}$ is untwisted.

Suppose that $M$ is minimally active and that $M \downarrow_{G_{0}}$ is the simple module $L(\lambda)$. By Lemma 10, either $L(\lambda)$ is (up to Frobenius twist) a $p$-restricted module, or $p=2$ and $G_{0}=\mathrm{SL}_{3}\left(2^{a}\right)$, or $p=2,3$ and $G_{0}=\mathrm{SL}_{2}\left(p^{a}\right)$, with $M$ a product of $p$ twists of the natural module. If $u$ induces a field automorphism, then $u$ replaces a highest weight $\lambda=a_{1} \lambda_{1}+\cdots+a_{n} \lambda_{n}$ with $p^{\alpha} a_{1} \lambda_{1}+\cdots+p^{\alpha} a_{n} \lambda_{n}$ for some $\alpha \geq 1$, and since graph automorphisms permute the $a_{i}$, for $u$ to fix $L(\lambda)$ it cannot be $p$-restricted. 
In the remaining cases of $\mathrm{SL}_{3}\left(2^{a}\right)$ and $\mathrm{SL}_{2}\left(p^{a}\right)$ for $p=2,3$, we have from Lemma 10 that $u$ has order $p$ and $M$ is the tensor product of the modules in a single orbit under the action of $u$ : for $G_{0}=\operatorname{SL}_{2}\left(p^{a}\right)$, this orbit is clear, whereas for $\mathrm{SL}_{3}\left(2^{a}\right)$ if $u$ is a pure field automorphism, we get that $L(1,0)$ and $L\left(2^{a}, 0\right)$ form an orbit, and if $u$ is the product of the field and the graph, it is $L(1,0)$ and $L\left(0,2^{a}\right)$, yielding the modules in the statement of the proposition.

We therefore have that $M \downarrow_{G_{0}}$ is not simple, and is the sum of $t$ simple factors $M_{1}, \ldots, M_{t}$, each stabilized by $u^{t}$. By Lemma $9, u^{t}$ acts on each $M_{i}$ with a single Jordan block, and since $u^{t}$ cannot act as a pure graph automorphism on the $M_{i}$, we must therefore have that $u^{t} \in G_{0}$, and so the conclusion of the proposition holds here as well.

Now suppose that $G_{0}$ is twisted, so that $u$ must be a field automorphism: if $M \downarrow_{G_{0}}$ is simple, then it cannot be $p$-restricted, so as with the untwisted case we get that $G_{0}$ is of type $A_{2}$ and we proceed similarly to the case of $\operatorname{SL}_{3}\left(2^{a}\right)$. If $M \downarrow_{G_{0}}$ is not simple, then we get the same proof as for the untwisted case, yielding the result above.

There are many examples of ( $\mathrm{v}$ ) above, and some that may appear to be but are not. For example the group ${ }^{3} D_{4}(3) .3$ with $M$ of dimension 24 , is the sum of three 8-dimensional modules permuted by the graph automorphism. Each 8-dimensional module for ${ }^{3} D_{4}(3)$ possesses minimally active elements by Proposition 19, but since the simple group of type $D$ does not contain an element acting with a single block of size 8 , there can be no minimally active $u$ for $M$.

As another negative example, we consider $G={ }^{2} G_{2}(3)$, which has a module $M$ of dimension 27 where the derived subgroup $G^{\prime}$ acts on $M$ as the sum of three blocks, and $G^{\prime}$ contains an element $v$ (of order 9) acting on each factor with a single Jordan block, but there is no element $u \in G$ such that $u^{3}=v$ (as then the Sylow 3-subgroup would be cyclic).

We should enumerate which possibilities from Proposition 19 actually have an element acting with a single block, so that they can be inputs into case (v) in Proposition 21. For the group $G_{0}=\mathrm{SL}_{2}\left(p^{a}\right)$, we have that $u$ acts as a single block on $L(i)$ for $i<p$, but acts as $(3,1)$ on $L\left(1+p^{i}\right)$. For $G_{0}$ of type $A,{ }^{2} A$, $B,{ }^{2} B$, and $C$, there is an element acting with a single Jordan block, but not for those of type $D$. We can include the 5-dimensional module for $\operatorname{Sp}_{4}\left(p^{a}\right)$ in those above via the isomorphism $B_{2} \cong C_{2}$, and we also have $G_{2}$ with the minimal module.

Hence, if $G_{0}=\mathrm{SU}_{n}\left(p^{p}\right)$ for some $n, p$ for example, one may form the group $G=\mathrm{SU}_{n}\left(p^{p}\right) \cdot p$, and let $u$ be an element of $g$ whose $p$ th power is the regular unipotent element of $G_{0}$. In this case the hypotheses of (v) are satisfied, so this is an example. 


\begin{tabular}{|c|c|c|c|}
\hline$G_{0}$ & $\begin{array}{r}\text { Largest 2-element } \\
\text { of } G \backslash G_{0}\end{array}$ & $G_{0}$ & $\begin{array}{r}\text { Largest 2-element } \\
\text { of } G \backslash G_{0}\end{array}$ \\
\hline$M_{12}$ & 4 & $\mathrm{He}$ & 16 \\
\hline$M_{22}$ & 8 & HN & 8 \\
\hline HS & 8 & $\mathrm{Fi}_{22}$ & 16 \\
\hline$J_{2}$ & 8 & $\mathrm{Fi}_{24}^{\prime}$ & 16 \\
\hline $\mathrm{McL}$ & 8 & ON & 8 \\
\hline Suz & 16 & $J_{3}$ & 8 \\
\hline
\end{tabular}

Table 2. Exponents of Sylow 2-subgroups of $\operatorname{Aut}\left(G_{0} / Z\left(G_{0}\right)\right)$ for $G_{0}$ a sporadic quasisimple group with non-trivial outer automorphism group.

\section{Sporadic groups}

In [4], all almost cyclic, and in particular minimally active elements were found for the case where $G_{0}$ is a central extension of a sporadic simple group, and where $G=G_{0}$. In this short section we deal with the case where $u$ induces an outer automorphism of $G_{0}$. Since $\left|\operatorname{Out}\left(G_{0}\right)\right| \leq 2$, we will always assume in this section that $p=2$.

We can easily determine the outer classes of 2-elements from [2], and in Table 2 we give the largest order of such a 2-element, with the obvious intent to use the formula $\operatorname{dim}(M) \leq \alpha(u) \cdot(o(u)-1)$ from Lemma 5 (iv). For this we also need estimates for $\alpha(u)$ and $\operatorname{dim}(M)$ : the latter appears in [15], and the former in [4, Theorem 3.1]. (This gives $\alpha\left(u^{2}\right)$, but of course $\alpha(u) \leq \alpha\left(u^{2}\right)$. If $o(u)=2$, then we can use [10, Table 1].)

We get the following lemma.

Lemma 22. Let $G_{0}$ be a sporadic simple group, and suppose that $u \in G \backslash G_{0}$ is a 2-element. If $o(u) \geq 8$, then $\alpha(u)=2$. If $o(u)=4$, then $\alpha(u) \leq 3$. If $o(u)=2$, then $\alpha(u) \leq 8$, with $\alpha(u) \leq 4$ if $G_{0}=J_{2}$.

Proof. From the tables in [2], we see that $u$ cannot square to $4 \mathrm{~A}$ when $G_{0}=\mathrm{HS}$, nor to $2 \mathrm{~A}$ when $G_{0}=\mathrm{Fi}_{22}$. If $G_{0}=J_{2}$, then the class $4 \mathrm{~B}$ squares to $2 \mathrm{~A}$, so we need to check how many conjugates generate $G$ in this case: a quick computer calculation shows that $\alpha(u)=2$ for this class.

If $o(u)=2$, then we use the bounds on $\alpha(G)$ given in [10].

Combining this information with Table 3 , we see that the only possibilities for $u$ acting minimally actively are that $G_{0}=M_{22}, J_{2}$. For $G=M_{22} .2$, the only can- 


\begin{tabular}{lrlr}
\hline$G_{0}$ & $\begin{array}{r}\text { Minimal non-trivial } \\
\text { simple module }\end{array}$ & $G_{0}$ & $\begin{array}{r}\text { Minimal non-trivial } \\
\text { simple module }\end{array}$ \\
\hline$M_{12}$ & 10 & $\mathrm{He}$ & 51 \\
$M_{22}$ & 10 & $\mathrm{HN}$ & 132 \\
$\mathrm{HS}$ & 20 & $\mathrm{Fi}_{22}$ & 78 \\
$J_{2}$ & 6 & $\mathrm{Fi}_{24}^{\prime}$ & 3,774 \\
$\mathrm{McL}$ & 22 & $\mathrm{ON}^{\prime}$ & 10,944 \\
$\mathrm{Suz}$ & 110 & $J_{3}$ & 78 \\
\hline
\end{tabular}

Table 3. Minimal dimensions of non-trivial representations of $G_{0}$.

didate simple module has dimension 10, and elements of order 8 act on this with type $(8,2)$, so this is not an example. For $G=J_{2} .2$, the 6-dimensional simple modules of $G_{0}$ are swapped by the outer automorphism, so that $\operatorname{dim}(M) \geq 12$. In order for $u$ to act minimally actively on this it must act as a single Jordan block by Lemma 9, but that is clearly impossible. The next smallest dimension is 28 (see [16, p. 102]) so there are no examples here either.

This proves that $u \in G_{0}$ in all cases, so we get the following proposition, proved in [4].

Proposition 23. Suppose that $G_{0}$ is a central extension of a sporadic simple group, and let $u$ be a p-element of $G$ such that $G=\left\langle G_{0}, u\right\rangle$. If $u$ acts minimally actively on a non-trivial simple module $M$, then $G=G_{0}$ and one of the following holds:

(i) $G=M_{11}, p=11, o(u)=11$ and $\operatorname{dim}(M)=9,11$ (one representation each) or $\operatorname{dim}(M)=10$ (two representations),

(ii) $G=M_{12}, p=11, o(u)=11$ and $\operatorname{dim}(M)=11$ (two representations),

(iii) $G=2 \cdot M_{12}, p=11, o(u)=11$ and $\operatorname{dim}(M)=10$ (two representations) or $\operatorname{dim}(M)=12$ (one representation),

(iv) $G=3 \cdot M_{22}, p=2, o(u)=8$ and $\operatorname{dim}(M)=6$ (two representations),

(v) $G=2 \cdot M_{22}, p=11, o(u)=11$ and $\operatorname{dim}(M)=10$ (two representations),

(vi) $G=M_{23}, p=23, o(u)=23$ and $\operatorname{dim}(M)=21$,

(vii) $G=M_{24}, p=23, o(u)=23$ and $\operatorname{dim}(M)=23$,

(viii) $G=J_{2}, p=2, o(u)=8$ and $\operatorname{dim}(M)=6$ (two representations) requires $\mathbb{F}_{4} \subseteq k$, 
(ix) $G=2 \cdot J_{2}, p=7, o(u)=7$ and $\operatorname{dim}(M)=6$ (two representations) requires $\mathbb{F}_{49} \subseteq k$,

(x) $G=6 \cdot$ Suz, $p=11,13, o(u)=p$ and $\operatorname{dim}(M)=12$ (two representations),

(xi) $G=\mathrm{Co}_{3}, p=23, o(u)=23$ and $\operatorname{dim}(M)=23$,

(xii) $G=\mathrm{Co}_{2}, p=23, o(u)=23$ and $\operatorname{dim}(M)=23$,

(xiii) $G=2 \cdot \mathrm{Co}_{1}, p=23, o(u)=23$ and $\operatorname{dim}(M)=24$,

(xiv) $G=J_{1}, p=11, o(u)=11$ and $\operatorname{dim}(M)=7$,

(xv) $G=3 \cdot J_{3}, p=2, o(u)=8$ and $\operatorname{dim}(M)=9$ (two representations),

(xvi) $G=3 \cdot J_{3}, p=17,19, o(u)=p$ and $\operatorname{dim}(M)=18$ (four representations),

(xvii) $G=2 \cdot \mathrm{Ru}, p=29, o(u)=29$ and $\operatorname{dim}(M)=28$ (two representations).

Unless specified, the field of definition of these representations is the smallest $k$ such that $\left|Z\left(G_{0}\right)\right|$ divides $\left|k^{\times}\right|$.

\section{Groups of Lie type in cross characteristic: preliminaries}

In this section we tackle groups of Lie type in characteristic $r$ where $r \neq p$, so let $q$ be a power of $r$. We specifically exclude the case where the group is also a group in characteristic $p$, so for $\mathrm{SL}_{3}(2)$ for example, $p \neq 2$, 7. If $G_{0}$ is a classical group and $M$ is a Weil representation, or if $G_{0}=\operatorname{PSL}_{2}(q)$, with $u$ inducing an inner-diagonal automorphism on $G_{0}$ in both cases, then all minimally active $u$ are classified in [5], but the case where $u$ induces particularly a non-diagonal outer automorphism on $M$ is missing. In this section we give some notation and introduce some previous results, particularly on minimal dimensions of irreducible representations.

Let $G_{0}$ be a quasisimple group of Lie type, defined over the field $\mathbb{F}_{q}$, let $r$ be the prime dividing $q$, and let $p \neq r$ be another prime. With a few exceptions given in [7, Table 6.1.3], $G_{0}$ is (a quotient of) the fixed points of a Frobenius endomorphism of a simple, simply connected algebraic group in characteristic $r$.

Note that those groups of Lie type that are isomorphic to alternating groups have already been covered in Proposition 16, and we will ignore them from now on. We will also ignore cases where $G_{0}$ is isomorphic to a group in characteristic $p$, so under the isomorphisms:

- $\mathrm{PSL}_{2}(4) \cong \mathrm{PSL}_{2}(5) \cong A_{5}$,

- $\mathrm{PSp}_{4}(2)^{\prime} \cong \mathrm{PSL}_{2}(9) \cong A_{6}$,

- $\mathrm{PSL}_{4}(2) \cong A_{8}$, 
- $\operatorname{PSL}_{3}(2) \cong \operatorname{PSL}_{2}(7)$,

- $\mathrm{PSU}_{3}(3) \cong G_{2}(2)^{\prime}$,

- $\mathrm{PSL}_{2}(8)={ }^{2} G_{2}(3)^{\prime}$,

- $\mathrm{PSU}_{4}(2) \cong \mathrm{PSp}_{4}(3)$.

We find all simple, minimally active modules for classical $G$ with $G_{0}$ possessing one of the central extensions given in [7, Table 6.1.3] now. For $G_{0}$ of exceptional type, see Proposition 36 except for ${ }^{2} E_{6}(2)$, for which standard arguments work.

In the next result, if there is more than one central extension $n \cdot G$ (for example, $G=\mathrm{PSL}_{3}$ (4) has more than one 4-fold extension), we use the numbering of these from the Atlas [2], and we use [16] for information about the existence of certain simple modules for central extensions.

Proposition 24. Let $G_{0}$ be a central extension of one of the following groups: $\mathrm{PSL}_{3}(2), \mathrm{PSL}_{3}(4), \mathrm{PSU}_{4}(2), \mathrm{PSU}_{4}(3), \mathrm{PSU}_{6}(2), \mathrm{Sp}_{6}(2), \Omega_{7}(3), \Omega_{8}^{+}(2)$. Let $u$ be a p-element of $G$ such that $G=\left\langle G_{0}, u\right\rangle$. If $u$ acts minimally actively on a nontrivial simple module $M$, then one of the following holds:

(i) $G=\mathrm{SL}_{3}(2), p=3, o(u)=3$ and $\operatorname{dim}(M)=3$ (two representations) requires $\mathbb{F}_{9} \subseteq k$,

(ii) $G=2 \cdot \mathrm{SL}_{3}(2), p=3, o(u)=3$ and $\operatorname{dim}(M)=4$ (two representations) requires $\mathbb{F}_{9} \subseteq k$,

(iii) $G=6 \cdot \mathrm{PSL}_{3}(4), p=5,7, o(u)=p$ and $\operatorname{dim}(M)=6$ (two representations), with $u$ acting as $(5,1)$ or $(6)$,

(iv) $G=4_{1} \cdot \mathrm{PSL}_{3}(4), p=7, o(u)=7$ and $\operatorname{dim}(M)=8$ (four representations), with $u$ acting as $(7,1)$,

(v) $G=\mathrm{PSU}_{4}(2), p=5, o(u)=5$ and $\operatorname{dim}(M)=5$ (two representations) requires $\mathbb{F}_{25} \subseteq k$ or $\operatorname{dim}(M)=6$ (one representation), with $u$ acting as (5) or $(5,1)$,

(vi) $G=2 \cdot \mathrm{PSU}_{4}(2), p=5, o(u)=5$ and $\operatorname{dim}(M)=4$ (two representations) requires $\mathbb{F}_{25} \subseteq k$, with $u$ acting as (4),

(vii) $G=66_{1} \cdot \mathrm{PSU}_{4}(3), p=5,7, o(u)=p$ and $\operatorname{dim}(M)=6$ (two representations), with $u$ acting as $(5,1)$ or $(6)$,

(viii) $G=31 \cdot \mathrm{PSU}_{4}(3), p=2, o(u)=8$ and $\operatorname{dim}(M)=6$ (two representations), with $u$ acting as (6), 
(ix) $G=3_{1} \cdot \mathrm{PSU}_{4}(3) .2_{2}, p=2$, $u$ can have order $2,4,8$ (but not all elements of orders 2 or 4 ), and $\operatorname{dim}(M)=6$ (two representations), with $u$ acting as $\left(2,1^{4}\right),\left(4,1^{2}\right)$ and $(6)$,

(x) $G=\operatorname{Sp}_{6}(2), p=3, o(u)=3,9$ and $\operatorname{dim}(M)=7$, with $u$ acting as $\left(3,1^{4}\right)$ or (7) (minimally active elements of order 3 are from the smallest conjugacy class),

(xi) $G=\operatorname{Sp}_{6}(2), p=5,7, o(u)=p$ and $\operatorname{dim}(M)=7$, with $u$ acting as $\left(5,1^{2}\right)$ and (7),

(xii) $G=2 \cdot \operatorname{Sp}_{6}(2), p=3,7, o(u)=7,9$ and $\operatorname{dim}(M)=8$, with $u$ acting as $(7,1)$,

(xiii) $G=2 \cdot \Omega_{8}^{+}(2), p=3$, $u$ has order 9 , or $u$ has order 3 with centralizer $Z_{6} \times \mathrm{PSp}_{4}(3)$, and $\operatorname{dim}(M)=8$,

(xiv) $G=2 \cdot \Omega_{8}^{+}(2), p=5$, $u$ has order 5 with centralizer $Z_{10} \times \mathrm{PSL}_{2}$ (5), and $\operatorname{dim}(M)=8$,

(xv) $G=2 \cdot \Omega_{8}^{+}(2), p=7, o(u)=7$ and $\operatorname{dim}(M)=8$.

Unless specified, the field of definition of these representations is the smallest $k$ such that $\left|Z\left(G_{0}\right)\right|$ divides $\left|k^{\times}\right|$.

Proof. If $G_{0}$ has $\mathrm{PSL}_{3}(2)$ as a quotient, then $p \neq 2,7$, as these were considered in Section 5. Thus $p=3, G=\mathrm{SL}_{2}(7), o(u)=3$, and $C_{G}(u)=\langle u\rangle \cdot Z(G)$, so that $M$ is minimally active if and only if $\operatorname{dim}(M) \leq p+1=4$ by Lemma 8 .

Now let $G_{0}$ be a central extension of $\operatorname{PSL}_{3}(4)$, and to begin let $p=3$. The Sylow 3-subgroup of $\mathrm{PGL}_{3}(4)$ has exponent 3: $\mathrm{PSL}_{3}(4)$ has a unique class of elements $u$ of order 3 and is generated by two conjugates of them, so if $u$ acts minimally actively, then $\operatorname{dim}(M) \leq 4$, but the minimal dimension for a simple module for $(4 \times 4) \cdot \mathrm{PSL}_{3}(4)$ is 6 . (The Schur multiplier of $\mathrm{PSL}_{3}(4)$ is $4 \times 4 \times 3$.)

Alternatively, $u$ could lie outside $\mathrm{PSL}_{3}(4)$ in $G$, and then $G$ is generated by three conjugates of $u$, $\operatorname{so} \operatorname{dim}(M) \leq 6$. However, we cannot form a central extension of $\mathrm{PGL}_{3}(4)$ by a 2-group as the outer automorphism acts transitively on the involutions in the $Z_{4} \times Z_{4}$ Sylow 2-subgroup of the Schur multiplier, and the minimal dimension for $\mathrm{PGL}_{3}(4)$ is 19.

When $p=5$, the normalizer of a Sylow 5-subgroup of $\mathrm{PSL}_{3}(4)$ is $D_{10}$, so we apply Lemma 8 to see that $M$ is minimally active if and only if $\operatorname{dim}(M) \leq 6$. Similarly, the normalizer of a Sylow 7-subgroup of $\mathrm{PSL}_{3}(4)$ is $Z_{7} \rtimes Z_{3}$, so again $\operatorname{dim}(M) \leq 8$ by Lemma 8 . There are 6-dimensional representations of $6 \cdot \operatorname{PSL}_{3}(4)$ modulo 5 and 7 , and 8-dimensional representations of $4_{1} \cdot \mathrm{PSL}_{3}(4)$.

If $G_{0}$ is a central extension of $\mathrm{PSU}_{4}(2)=\mathrm{PSp}_{4}(3)$, then $p=5$. For $p=5$, the Sylow 5-subgroup has order 5, generated by $u$, and $C_{G}(u)=\langle u\rangle \cdot Z(G)$, so 
that $M$ is minimally active if and only if $\operatorname{dim}(M) \leq p+1=6$. There are modules for $\mathrm{PSU}_{4}(2)$ of dimensions 5 and 6 , and of $2 \cdot \mathrm{PSU}_{4}(2)$ of dimension 4 , completing the proof.

Let $G_{0}$ be a central extension of $\mathrm{PSU}_{4}(3)$, so that $p=2,5,7$. For $p=5,7$, the Sylow $p$-subgroup is of order $p$, generated by $u$, and $C_{G}(u)=\langle u\rangle \cdot Z(G)$, so that $\operatorname{dim}(M) \leq p+1$ by Lemma 8 . There is a module of dimension 6 for $6_{1} \cdot \mathrm{PSU}_{4}(3)$, so this is minimally active for both primes. For $p=2$, as $\operatorname{Out}\left(G_{0}\right)$ is $D_{8}$ and the Schur multiplier is $3 \times 3 \times 4$ there are many potential groups $G$.

If $G_{0}=\operatorname{PSU}_{4}(3)$, then from [16] we see that $\operatorname{dim}(M)=20$ or $\operatorname{dim}(M) \geq 34$. The order of $u \in G$ is 2, 4, 8 from [2], and for $u \in G_{0}, \alpha(u)=2$ if $o(u)=4,8$, with $\alpha(u)=3$ if $o(u)=2$. As $\operatorname{Out}\left(G_{0}\right)=D_{8}, u^{4} \in G_{0}$, so the only way that $u$ can act minimally actively is if $o(u)=8, u^{2} \notin G_{0}$, and $\alpha(u)=3$. However, by constructing $\operatorname{Aut}\left(G_{0}\right)$ in Magma, we check that $\alpha(u)=2$ for all $u$ of order 8 , hence there are no non-trivial minimally active modules if $Z\left(G_{0}\right)=1$. In $\operatorname{Aut}\left(G_{0}\right)$, we have $\alpha(u)=2$ if $o(u)=8, \alpha(u) \leq 4$ if $o(u)=4$, and $\alpha(u) \leq 6$ if $o(u)=2$.

Thus $G_{0}$ is either $3_{1} \cdot \mathrm{PSU}_{4}(3)$ or $3_{2} \cdot \mathrm{PSU}_{4}(3)$. In the second case, we have $\operatorname{dim}(M) \geq 36$, so we again see that there are no minimally active modules by the above computations for $\alpha(u)$, as $\operatorname{dim}(M) \leq 14$ for $M$ to be minimally active. Thus $G_{0}=3_{1} \cdot \mathrm{PSU}_{4}(3)$, and from [2, p. 53] we see that the only outer automorphism that centralizes $G_{0}$ is $2_{2}$, so we let $G$ be either $G_{0}$ or $G_{0} .2_{2}$.

The only non-trivial simple module for $G_{0}$ of dimension at most 14 has dimension 6 (two up to duality), and this extends to $G_{0} \cdot 2_{2}$. Inside $G_{0}$ elements of order 8 act with type (6), and in $G_{0} .2_{2}$ (modulo a central involution, this is the complex reflection group $G_{34}$ in Shephard-Todd notation) there are elements of orders 2, 4 and 8 (with the last one not in $\left.G_{0}\right)$ that act with types $\left(2,1^{4}\right),\left(4,1^{2}\right)$ and (6), respectively.

Let $G_{0}$ be a central extension of $\operatorname{PSU}_{6}(2)$, so that $p=3,5,7,11$. If $p=7,11$, then $C_{G_{0}}(u)=\langle u\rangle \cdot Z\left(G_{0}\right)$, so that $\operatorname{dim}(M) \leq p+1$ if and only if $M$ is minimally active by Lemma 8 . However, $\operatorname{dim}(M) \geq 21$ for all odd $p$ by [21], a contradiction. If $p=5$, then $o(u)=5$ and $\alpha(u)=2$, so that $\operatorname{dim}(M) \leq 8$ for minimally active $M$, another contradiction. If $p=3$, then there is an outer automorphism of order $p$, but in $\mathrm{PGU}_{6}(2)$ the exponent of the Sylow 3-subgroup is still 9, so if $\alpha(u)=2$ when $o(u)=9$ and $\alpha(u) \leq 10$ for $o(u)=3$, then we are done. The former follows by a computer calculation, and the latter follows from [10, Theorem 4.1].

Let $G_{0}$ be a central extension of $\operatorname{Sp}_{6}(2)$, so that $p=3,5,7$. The Sylow 7-subgroup of $G_{0}$ is cyclic, and if $u$ is a generator for it, then $C_{G_{0}}(u)=\langle u\rangle \cdot Z(G)$, so that $\operatorname{dim}(M) \leq p+1=8$ if and only if $M$ is minimally active. There is a 7-dimensional simple module for $\mathrm{Sp}_{6}(2)$ and an 8-dimensional simple module for $2 \cdot \operatorname{Sp}_{6}(2)$, so these are minimally active. If $p=5$, then the Sylow 5-subgroup $P$ 
has order 5 , and $\alpha(u)=2$ for $u$ of order 5 , yielding $\operatorname{dim}(M) \leq 8$ for minimally active modules. The module of dimension 7 is minimally active, with $u$ of type $\left(5,1^{2}\right)$, but on the module of dimension 8 the action has type $\left(4^{2}\right)$.

For $p=3$, if $o(u)=9$, then $\alpha(u)=2$, and if $o(u)=3$, then $\alpha(u) \leq 4$, so $\operatorname{dim}(M) \leq 16$ for $M$ minimally active. There are three such non-trivial simple modules, of dimensions 7, 8 and 14 . The 14-dimensional module is not minimally active, but elements of order 9 act with a single Jordan block on the other two modules. In addition, elements of order 3 from the smallest class, with centralizer of order 2160 in $\mathrm{Sp}_{6}(2)$, act on $M$ of dimension 7 with type $\left(3,1^{4}\right)$.

The next group is $G_{0}$ a central extension of $\Omega_{7}(3)$, with primes $2,5,7,13$. From [21], $\operatorname{dim}(M) \geq 27$ if $M$ is non-trivial. Note that $o(u) \leq 13$, and $\alpha(u)=2$ if $o(u) \geq 5, \alpha(u) \leq 3$ for $o(u)=4, \alpha(u) \leq 4$ if $o(u)=3$ and $\alpha(u) \leq 7$ if $o(u)=2$. Thus there are no minimally active modules for $G_{0}$. However, $\operatorname{Out}\left(G_{0}\right)$ has order 2 , and from [2] we see that the exponent of the Sylow 2-subgroup of $\operatorname{Aut}\left(\Omega_{7}(3)\right)$ has order 8; in this case, $\alpha(u)$ is as before if $o(u)=2,8$, and $\alpha(u) \leq 4$ if $o(u)=4$. Again, there can be no non-trivial simple minimally active modules.

The final group on our list is $\Omega_{8}^{+}(2)$, where $p=3,5,7$. For $G=2 \cdot \Omega_{8}^{+}(2)$, $\alpha(u)=2$ for $o(u)=5,7,9$, and $\alpha(u) \leq 4$ for $o(u)=3$. The 8-dimensional simple module for $G$ is minimally active for $p=7$, with type $(7,1)$, and for $p=5$ the three classes of elements of order 5 have type $(5,1,1,1)$, and $(4,4)$ twice, with the two classes of elements of order 5 having centralizer $Z_{5} \times \mathrm{SL}_{2}(5)$ and the minimally active one having centralizer $Z_{10} \times \mathrm{PSL}_{2}(5)$.

For $p=3$, we need to consider $G=2 \cdot \Omega_{8}^{+}(2)$ and $\Omega_{8}^{+}(2) .3$ : the former case is easy, with elements of order 9 acting on the 8 -dimensional module as $(7,1)$, and one of the three classes of elements of order 3 with centralizer of order 155,520 have type $\left(3,1^{5}\right)$ and the other two having type $\left(2^{4}\right)$; again these two have centralizer $Z_{3} \times \mathrm{Sp}_{4}$ (3), and the one we want has centralizer $Z_{6} \times \mathrm{PSp}_{4}$ (3). (There are two classes of elements of order 3 with smaller centralizer.)

To deal with $\Omega_{8}^{+}(2) .3$, note that from [16] we get that $\operatorname{dim}(M) \geq 28$, and $o(u)=3$, 9. Since $\alpha(u) \leq 4$ by [10, Theorem 4.4], elements of order 3 cannot work, and elements $u$ of order 9 must cube to an element $v=u^{3}$ of order 3 in $G_{0}$ that acts on $M$ with at most three blocks of size 3 . Since $\operatorname{dim}(M)=28$ or $\operatorname{dim}(M) \geq 48$, we have $\operatorname{dim}(M)=28$, and the five conjugacy classes of elements of order 3 act on $M$ as $\left(3^{6}, 1^{10}\right),\left(3^{7}, 2^{2}, 1^{3}\right)$ and $\left(3^{9}, 1\right)$, so it cannot be minimally active. This completes the proof for $\Omega_{8}^{+}(2)$.

Because of Proposition 24, if $G$ is classical, then we may take $G_{0}$ to be a quotient of one of the groups $\operatorname{SL}_{n}(q), \operatorname{SU}_{n}(q), \operatorname{Sp}_{2 n}(q), \operatorname{Spin}_{2 n+1}(q)$ and $\operatorname{Spin}_{2 n}^{ \pm}(q)$. The order of $G_{0}(q)$ is given by a polynomial $q^{N} \prod_{i} \Phi_{i}(q)^{a_{i}}$, where $N$ and the $a_{i}$ are integers, and $\Phi_{i}$ denotes the $i$ th cyclotomic polynomial. If $u$ is a $p$-element 
and $p \nmid q$, then $p$ divides one of the $\Phi_{i}(q)$; let $d$ denote the order of $q$ modulo $p$, so that $p \mid \Phi_{d}(q)$ and $p \nmid \Phi_{e}(q)$ for all $1 \leq e<d$. (If $p=2$, we let $d$ be the order of $q$ modulo 4.) This next well-known lemma tells us about the powers of $p$ dividing various cyclotomic polynomials.

Lemma 25. Let $p \neq r$ be a prime and suppose that $q$ is a power of $r$.

(i) Writing $d$ for the order of $q$ modulo $p, p \mid \Phi_{e}(q)$ if and only if $e=p^{a} d$ for some $a \geq 0$ (except if $p=2$ and $d=2$, where $2 \mid \Phi_{1}(q)$ as well).

(ii) If $e$ is not the order of $q$ modulo $p$, then $p^{2} \nmid \Phi_{e}(q)$.

(iii) We have

$$
\Phi_{d}\left(q^{p}\right)= \begin{cases}\Phi_{d}(q) \cdot \Phi_{p d}(q) & \text { if } p \nmid d, \\ \Phi_{p d}(q) & \text { if } p \mid d .\end{cases}
$$

Therefore for all $d$, the powers of $p$ dividing $\Phi_{d}\left(q^{p^{a}}\right)$ and $p^{a} \Phi_{d}(q)$ are the same.

We now need information about the cross-characteristic Sylow structure of a group of Lie type, which is described in [7, Theorem 4.10.2]. We give a summary now, tailored to our needs.

Proposition 26. Let $G_{0}=G_{0}(q)$ denote a quasisimple group of Lie type, with $Z\left(G_{0}\right)$ a $p^{\prime}$-group. Let d denote the order of $q$ modulo $p$, and let $p^{a}$ be the exact power of $p$ dividing $\Phi_{d}(q)$. Let $P$ be a Sylow p-subgroup of $G_{0}$. There exists an abelian normal subgroup $P_{0}$ of $G_{0}$, of exponent $p^{a}$, such that $P / P_{0}$ is isomorphic to a subgroup of the Weyl group of $G_{0}$, unless one of the following holds:

(i) $p=3, G_{0}={ }^{3} D_{4}(q)$, where $P_{0}$ has exponent $p^{a+1}$,

(ii) $p=2, G_{0}={ }^{2} G_{2}(q)$, where $P$ is elementary abelian of order 8 .

Furthermore, if $G$ is an almost simple group containing $G_{0}$ as a normal subgroup, with $G / G_{0}$ consisting of diagonal automorphisms, then the same results hold.

This means that, in the notation of the proposition, if the exponent of the Sylow $p$-subgroup of the Weyl group of $G_{0}$ is $p^{b}$, then the exponent of $P$ is at most $p^{a+b}$ (except in the one case, where it is at most $p^{a+b+1}$ ).

To get a bound for the maximal order of $u$, we finally need to consider the outer automorphism group of $G_{0}$, which is more or less completely described in [7, Theorem 2.5.12]. Thus the contribution to $o(u)$ comes from three sources: the toral contribution, the $p$-part of $\Phi_{d}(q)$ (except for ${ }^{3} D_{4}$ ), the Weyl contribution, the exponent of the Sylow $p$-subgroup of the Weyl group, and the outer contribution, the exponent of the Sylow $p$-subgroup of the outer automorphism group 
of $G_{0}$. This is usually far greater than the actual maximal order of $u$, and so we use this to reduce the possible options for $G_{0}$, and then use more explicit techniques to get better bounds on $o(u)$ if required.

Notice that the Sylow $p$-subgroup of $G_{0}$ is abelian if and only if $p$ divides exactly one of the $\Phi_{d}(q)$ that divide $\left|G_{0}\right|$, or in other words, if the Sylow $p$-subgroup of $G_{0}$ is non-abelian and the order of $q$ modulo $p$ is $d$, then both $\Phi_{d}(q)$ and $\Phi_{p d}(q)$ divide $\left|G_{0}(q)\right|$.

We also need to consider regular semisimple elements, in particular to know that their centralizer is abelian when $u$ is a regular semisimple element in a cyclic Sylow subgroup. This result appears in [3, Proposition 9.1]. We remind the reader of the definition of a regular number. If $W$ is a Coxeter group, a regular element is an element that acts regularly on the reflection representation, and a regular number is an number that is the order of a regular element. These are enumerated in $[28$, Section 5].

Lemma 27. Let $p$ be a prime and let $q$ be a power of a prime $r \neq p$, and let $G(q)$ be a finite group of Lie type. Suppose that the order of $q$ modulo $p$ is a regular number, and that the Sylow p-subgroup of $G(q)$ is cyclic. If $u$ is a generator for the Sylow p-subgroup of $G(q)$, then the centralizer $C_{G}(u)$ is abelian.

\section{Classical groups in cross characteristic}

In this section we consider the case where $G_{0}$ is a central extension of a classical group in characteristic $r \neq p$.

In [5], Di Martino and Zalesski solve the problem of which elements of quasisimple classical groups act minimally actively on the Weil modules (in fact, they do all almost cyclic elements). However, they only allow $u$ to induce a innerdiagonal outer automorphism on $G_{0}$ if it is linear or unitary, and only an inner automorphism if $G_{0}$ is symplectic. The theorem in [5], applied to minimally active modules only (i.e., where $u$ is a $p$-element and the characteristic of the field is $p$ ), is as follows.

Theorem 28. Let $G_{0}$ be one of $\mathrm{SL}_{n}(q)(n \geq 3), \mathrm{SU}_{n}(q)$ or $\mathrm{Sp}_{2 n}(q)$, and let $u$ either be in $G_{0}$ or induce an inner-diagonal automorphism on $G_{0}$ if $G_{0}$ is not symplectic. Suppose that $G_{0}$ is not one of the groups considered in Proposition 24. If $u$ acts minimally actively on a Weil module, then one of the following holds:

(i) $G=\operatorname{Sp}_{2 n}(q), n$ is a power of $2, p^{a}=\frac{q^{n}+1}{2}$ for some $a \geq 1, o(u)=p^{a}$,

(ii) $G=\mathrm{Sp}_{2 n}$ (3), $n \neq p$ is an odd prime and $p^{a}=\frac{3^{n}-1}{2}$ for some $a \geq 1$, with $o(u)=p^{a}$, 
(iii) $G_{0}=\mathrm{SU}_{n}(q), n \neq p$ is an odd prime at least $5, p^{a}=\frac{q^{n}+1}{q+1}$ for some $a \geq 1$, and $o(u)=p^{a}$,

(iv) $G=\mathrm{SU}_{3}(3), p=7, o(u)=7$,

(v) $G_{0}=\operatorname{SL}_{n}(q), n \neq p$ is an odd prime, $p^{a}=\frac{q^{n}-1}{q-1}$ for some $a \geq 1$, and $o(u)=p^{a}$.

We will add to this by proving the following result.

Proposition 29. Let $G_{0}$ be a central extension of a simple special linear, unitary or symplectic group, but not one of the groups in Proposition 24. Let $u \in G$ be a p-element, and let $M$ be a simple module on which u acts minimally actively. If $M \downarrow_{G_{0}}$ involves a Weil module, then $u$ induces an inner-diagonal automorphism on $G_{0}$.

We begin by proving, for $G_{0}$ classical and not of type $\mathrm{PSL}_{2}$, that if $M$ is not a Weil module, then the possibilities for an element of $G$ acting minimally actively are very limited, restricted mostly to cases of exceptional Schur multipliers given in Proposition 24.

Table 4 is a summary of what we will need about the dimensions of Weil modules, and lower bounds for the dimensions of non-Weil modules for classical groups, assuming that $G_{0}$ is not one of the groups in Proposition 24. (Let $\kappa_{n}$ be 1 if $p$ divides $\frac{q^{n}-1}{q-1}$ and 0 otherwise.)

\subsection{Groups $\operatorname{SL}_{n}(q), n \geq 3$}

For this subsection we let $G_{0}$ be a quotient of $\operatorname{SL}_{n}(q)$ for $n \geq 3$, and we exclude the cases of $\mathrm{PSL}_{3}(2)=\mathrm{PSL}_{2}(7), \mathrm{PSL}_{4}(2)=\mathrm{Alt}_{8}$, and $\mathrm{PSL}_{3}$ (4) which are considered in Propositions 16 and 24. Suppose that $M$ is a non-trivial simple module, but not a Weil module. From Table 4, the dimension of $M$ is at least

$$
\left(q^{n-1}-1\right)\left(\frac{q^{n-2}-1}{q-1}-1\right)
$$

for $n \geq 5$.

Note that if $u$ is a $p$-element of $G$, then, as we saw in Proposition 26 and the discussion afterwards, the order of $u$ is bounded by a product of numbers: the exponent of the Sylow $p$-subgroup of the outer automorphism group (the outer contribution); the exponent of the Sylow $p$-subgroup of the Weyl group (only if the Sylow $p$-subgroup of $G_{0}$ is non-abelian, the Weyl contribution); the $p$-part of $\Phi_{d}(q)$ (the toral contribution).

We will let $G_{0}$ be a group $G\left(q^{t}\right)$ and assume that $u$ induces an automorphism on $G_{0}$ that projects onto a field automorphism of order $t$ in $\operatorname{Out}\left(G_{0}\right)$. 


\begin{tabular}{lll}
\hline Group & Bound & Reference \\
\hline $\mathrm{SL}_{n}(q)$ (Weil) & $\frac{q^{n}-q}{q-1}-\kappa_{n}$ & {$[11]$} \\
$\mathrm{SL}_{n}(q)$ (Weil) & $\frac{q^{n}-1}{q-1}$ & {$[11]$} \\
$\mathrm{SL}_{n}(q), n=3,4$ (non-Weil) & $\frac{(q-1)\left(q^{n-1}-1\right)}{\text { gcd }(n, q-1)}$ & {$[11]$} \\
$\mathrm{SL}_{n}(q), n \geq 5$ (non-Weil) & $\left(q^{n-1}-1\right)\left(\frac{q^{n-2}-q}{q-1}-\kappa_{n-2}\right)$ & {$[11]$} \\
$\mathrm{SU}_{n}(q)$ (Weil) & $\frac{q^{n}+q(-1)^{n}}{q+1}$ & {$[12]$} \\
$\mathrm{SU}_{n}(q)$ (Weil) & $\frac{q^{n}-(-1)^{n}}{q+1}$ & {$[12]$} \\
$\mathrm{SU}_{3}(q)($ non-Weil) & $\frac{(q-1)\left(q^{2}+3 q+2\right)}{6}$ & {$[12]$} \\
$\mathrm{SU}_{4}(q)$ (non-Weil) & $\frac{\left(q^{2}+1\right)\left(q^{2}-q+1\right)}{2}-1$ & {$[12]$} \\
$\mathrm{SU}_{n}(q), n \geq 5$ (non-Weil) & $\frac{q^{n-2}(q-1)\left(q^{n-2}-q\right)}{q+1}$ & {$[12]$} \\
$\operatorname{Sp}_{2 n}(q), q$ odd (Weil) & $\frac{q^{n} \pm 1}{2}$ & {$[9]$} \\
$\mathrm{Sp}_{2 n}(q)$, all $q$ (non-Weil) & $\frac{q\left(q^{n}-1\right)\left(q^{n-1}-1\right)}{2(q+1)}$ & {$[9],[27]$} \\
$\Omega_{2 n+1}(q)$ & $q^{n-1}\left(q^{n-1}-1\right)$ & {$[13]$} \\
$\Omega_{2 n}^{+}(q)$ & $q^{n-2}\left(q^{n-1}-1\right)$ & {$[21]$} \\
$\Omega_{2 n}^{-}(q)$ & $\left(q^{n-1}+1\right)\left(q^{n-2}-1\right)$ & {$[21]$} \\
\hline
\end{tabular}

Table 4. Minimal dimension of a non-trivial projective representation for simple classical groups.

Proposition 30. Suppose that $G_{0}$ is a central extension of a special linear group $\mathrm{PSL}_{n}\left(q^{t}\right)$ for some $n \geq 3$, with $\left(n, q^{t}\right) \neq(3,2),(3,4),(4,2)$, and let $u$ be a $p$-element of $G$ such that $G=\left\langle G_{0}, u\right\rangle$. If $u$ acts minimally actively on a non-trivial simple module $M$ that is not $a$ Weil module, then $G=\mathrm{PSL}_{3}(3), p=13, o(u)=13$ and $\operatorname{dim}(M)=11,13$.

Proof. Let $G_{0}$ be a central extension of $\operatorname{PSL}_{n}\left(q^{t}\right)$ for some $n \geq 3$ and $t \geq 1$, with the exclusions given above of $\mathrm{PSL}_{3}(2), \mathrm{PSL}_{3}(4)$ and $\mathrm{PSL}_{4}(2)$. First, let $n=3,4$, and note that if $M$ is a non-Weil simple module, then

$$
\operatorname{dim}(M) \geq \frac{\left(q^{t}-1\right)\left(q^{(n-1) t}-1\right)}{\operatorname{gcd}\left(n, q^{t}-1\right)}
$$

If the Sylow $p$-subgroup of $\mathrm{SL}_{n}\left(q^{t}\right)$ is abelian, then, in the notation introduced after Proposition 26, the Weyl contribution is 1, the toral contribution is at most 
$\Phi_{d}\left(q^{t}\right)$, where $d=1, \ldots, n$, and the outer contribution is $t$, since diagonal automorphisms are not of concern by Proposition 26. Thus in all cases, we have that $o(u) \leq \Phi_{3}\left(q^{t}\right) \cdot t$, in fact $o(u) \leq \Phi_{3}(q) \cdot t^{2}$ by Lemma 25. From [10, Theorem 4.1], $\alpha(u) \leq n$ for $p>2$, and we have a lower bound for $\operatorname{dim}(M)$ from Table 4 . The equation $\operatorname{dim}(M) \leq \alpha(u) \cdot(o(u)-1)$ for minimally active $M$ from Lemma 5 (iv) now yields

$$
\frac{\left(q^{t}-1\right)\left(q^{(n-1) t}-1\right)}{\operatorname{gcd}\left(n, q^{t}-1\right)} \leq n\left(\left(q^{2}+q+1\right) t^{2}-1\right)
$$

since $p \mid t$, we can assume that $t$ is odd, and all solutions are for $t=1$, with $q=2,3,4,7$ for $n=3$ and $q=2,3$ for $n=4$. Removing those excluded from the start of this section, for $t=1$ we need consider $(n, q)=(3,3),(3,7),(4,3)$. For $\mathrm{PSL}_{3}(7)$, the only prime for which the Sylow $p$-subgroup is abelian is $p=19$, $\operatorname{dim}(M) \geq 96$ (by Table 4) and $\alpha(u) \leq 3$, so it in fact fails the bound. For PSL 4 (3), $\operatorname{dim}(M) \geq 26$ (again, table 4) and $p=5,13$, with $o(u)=p$. Since $\alpha(u) \leq 4$, this shows that $p=5$ cannot yield a minimally active module, and for $p=13$ we see that $C_{G_{0}}(u)=Z\left(G_{0}\right) \cdot\langle u\rangle$, so that $\operatorname{dim}(M) \leq p+1$ for $M$ to be minimally active by Lemma 8 . Thus there are no examples here.

For $\operatorname{PSL}_{3}(3)$ we have $p=13$, and again $C_{G_{0}}(u)=\langle u\rangle$ (there are no central extensions) so we may apply Lemma 8. The simple modules from [16] have dimensions $1,11,13,16$ and 26, so those of dimensions 11 and 13 are minimally active.

Suppose that the Sylow $p$-subgroups are non-abelian, so that $p=2,3$. First assume $p=3$ : the exponent of the Sylow 3-subgroup of the Weyl group is 3, and the toral contribution of $o(u)$ is at most $q^{t}+1$, in fact $t(q+1)$ by Lemma 25 . The outer contribution is at most $t$, so that $o(u) \leq 3 t^{2}(q+1)$. Thus our equation $\operatorname{dim}(M)<\alpha(u) \cdot o(u)$ becomes

$$
\frac{\left(q^{t}-1\right)\left(q^{(n-1) t}-1\right)}{\operatorname{gcd}\left(n, q^{t}-1\right)}<3 n t^{2}(q+1)
$$

which yields only $t=1$ and $(n, q)=(3,2),(3,4),(4,2)$, all of which are excluded.

For $p=2$, we have a graph automorphism to consider as well. The toral contribution to $o(u)$ is at most $q+1$, the Weyl contribution is at most $n$ and the outer contribution if the lowest common multiple of 2 and $t$.

Hence $\alpha(u) \cdot o(u)$ is at most $n m t(q+1) \cdot \operatorname{lcm}(2, t)$, where $m=n$ for $n \geq 5$, $m=4$ for $n=3$ and $m=6$ for $n=4$. We will check both Weil and non-Weil modules simultaneously, and all $n$, so we need

$$
n m t(q+1) \cdot \operatorname{lcm}(2, t) \geq \alpha(u) \cdot o(u)>\operatorname{dim}(M) \geq \frac{q^{n t}-q^{t}}{q^{t}-1}-1 .
$$


For $n \geq 5$ we only get $(n, q, t)=(5,3,1)$. For $n=4$ we get $(n, q, t)=(4,3,1)$, $(4,5,1)$, and for $n=3$ we get $q^{t} \leq 23$.

For $\operatorname{PSL}_{5}$ (3), the exponent of the Sylow 2-subgroup is 16 , so that $o(u) \leq 32$, $\alpha(u) \leq 5$, and $\operatorname{dim}(M) \geq 120$. Thus we need $o(u)=32$, and in this case $v=u^{2}$ is an element of $\mathrm{PSL}_{5}(3)$ of order 16 . However, a simple computer check confirms that $\alpha(v)=2$ for these elements, so that there is no minimally active module.

For $\mathrm{PSL}_{4}(3)$ and $\mathrm{PSL}_{4}(5)$, we have $\alpha(u) \leq 4$ if $\langle u\rangle \cap G_{0}$, generated by $v$ say, is non-trivial. Since the exponent of a Sylow 2-subgroup of both groups is 8 , we get that $o(u) \leq 16$, and $\operatorname{dim}(M) \geq 26,124$, respectively. This eliminates $\mathrm{PSL}_{4}(5)$ as $\operatorname{dim}(M)>o(u) \cdot \alpha(u)$ (using Lemma 5 (iv)). For $\mathrm{PSL}_{4}(3)$, if $o(v)=8$, then $\alpha(v)=2$ and if $o(v)=4$, then $\alpha(v) \leq 3$. Thus we can only get a minimal action of $u$ on $M$ if $\operatorname{dim}(M)=26$ (the next smallest is dimension 38) and $o(u)=16$, with $u$ therefore inducing the graph automorphism on $M$. However, there is no element of Aut $\left(\mathrm{PSL}_{4}(3)\right)$ of order 16, as we see from [2, pp. 68-69]. Thus we get no minimally active modules here either.

Finally, consider $n=3$. If $t=1$, then $\alpha(u) \leq 3$, and also the Weyl contribution to $o(u)$ is 2 , not $n$ which is 3 . Thus in this case we get $o(u) \cdot \alpha(u)$ to be at most $12(q+1)$, which yields $q \leq 13$ for there to be a minimally active module. If we replace $q+1$ by the 2-part of $\frac{q^{2}-1}{2}$, which is the actual toral contribution, we obtain $q \leq 9$. For these groups we check in [2] that the exponents of the Sylow 2-subgroups of $\operatorname{Aut}\left(\operatorname{PSL}_{3}(q)\right)$ are 8, 8, 16, 16, as $q=3,5,7,9$, respectively. Since $\operatorname{dim}(M) \geq 12,30,56,90$ from the table above, and $\alpha(u) \leq 3$, we see that in fact $G_{0}=\mathrm{PSL}_{3}(3)$ is the only possibility. In this case, if $u \in G_{0} .2$ has order 4 or 8 , we use a computer to check that $\alpha(u)=2$, and if $u \in G_{0} .2$ has order 2, then $\alpha(u) \leq 4$ by [10, Theorem 4.1]. Since if $M$ is minimally active then $\operatorname{dim}(M) \leq \alpha(u) \cdot(o(u)-1)$, we see that $\operatorname{dim}(M) \leq 14$, so the 12-dimensional simple module is the only possibility, with $o(u)=8$ (the simple modules for $G_{0}$ have dimensions 1, 12, 16 and 26). However, both classes of elements of order 8 in $G_{0} .2$ have type $(8,4)$ on $M$ of dimension 12 , so it is not minimally active.

Thus let $n \geq 5$ and $p$ be odd. If $M$ is not a Weil module, then

$$
\operatorname{dim}(M) \geq\left(q^{(n-1) t}-1\right)\left(\frac{q^{(n-2) t}-q^{t}}{q^{t}-1}-1\right),
$$

and for all $u, \alpha(u) \leq n$. If the Sylow $p$-subgroup of $G_{0}$ is abelian, then $o(u)$ is at most $\left(q^{n t}-1\right) /(q-1) \cdot t$, and using the formula $\operatorname{dim}(M)<o(u) \cdot \alpha(u)$ yields only $\operatorname{PSL}_{5}(2)$. As $\operatorname{dim}(M) \geq 75$ for non-Weil modules from the formula above, and the order of prime-power elements of $\mathrm{PSL}_{5}(2)$ is at most 8 or 31 , we see that $o(u)=31=\Phi_{5}(2)$. But in this case $C_{G_{0}}(u)=\langle u\rangle$, so that $\operatorname{dim}(M) \leq p+1=32$, and there are therefore no examples. 
Thus the Sylow $p$-subgroup of $G_{0}$ is non-abelian, and therefore $p$ divides two separate $\Phi_{d}$-tori: from Lemma 25 , we see therefore that if $q^{t}$ has order $d$ modulo $p, d p \leq n$, and therefore the toral contribution to $u$ is at most $q^{n t / p}-1$, with the Weyl contribution at most $n$ and the outer contribution $t$. We therefore have that $o(u) \leq n t\left(q^{\lfloor n t / 3\rfloor}-1\right)$ (as $p \geq 3$ ), and using the formula yields

$$
n^{2} t\left(q^{\lfloor n t / 3\rfloor}-1\right)>\left(q^{(n-1) t}-1\right)\left(\frac{q^{(n-2) t}-q^{t}}{q^{t}-1}-1\right),
$$

which has no solutions for $n \geq 5$. This completes the proof.

Having determined which non-Weil modules can be minimally active, we turn our attention to the Weil modules for odd primes $p$, where $u$ induces a nondiagonal outer automorphism, which must involve a field automorphism of order at least 3.

As in the proof of the previous proposition, the order of an element of $G_{0}$ is at most either $t\left(q^{n}-1\right)$ or $\operatorname{tn}\left(q^{\lfloor n / 3\rfloor}-1\right)$, and we multiply this by the outer contribution, which is $t$, and $\alpha(u)$, which is at most $n$, to get an estimate for $o(u) \cdot \alpha(u)$. We then compare that to $\left(q^{n t}-q^{t}\right) /\left(q^{t}-1\right)$ for $t \geq 3$, and find only one possible solution: $\mathrm{PSL}_{3}(8) .3$, of course with $p=3$. In this case, $\operatorname{dim}(M) \geq 72$ by [16, p. 187], and $o(u) \leq 9$ by [2, p. 74], so this cannot work either.

Thus if $u \in G$ acts minimally actively on a Weil module, then it induces a innerdiagonal automorphism on $G_{0}$, proving Proposition 29 for linear groups.

\subsection{Groups $\mathrm{SU}_{n}(q)$}

This looks very similar to the linear case in the previous subsection. We start by dealing with non-Weil representations, with the cases $n=3,4$ having to be dealt with separately, and at the same time proving that there are no minimally active modules for $p=2$, Weil or non-Weil. This then allows us to prove easily that $u$ cannot act minimally actively on a simple module without inducing an innerdiagonal automorphism on $G_{0}$, just as with the linear case.

Note that we exclude $\operatorname{PSU}_{n}(q)$ for $(n, q)=(3,2),(4,2),(4,3),(6,2)$. For $\operatorname{PSU}_{3}(3)=G_{2}(2)^{\prime}$, we require $p \neq 2,3$.

Proposition 31. Suppose that $G_{0}$ is a central extension of a special unitary group $\operatorname{PSU}_{n}\left(q^{t}\right)$ for some $n \geq 3$, with $\left(n, q^{t}\right) \neq(3,2),(4,2),(4,3),(6,2)$, and let $u$ be a p-element of $G$ such that $G=\left\langle G_{0}, u\right\rangle$. If $u$ acts minimally actively on a nontrivial simple module $M$, then $M$ is a Weil module.

Proof. Let $G_{0}$ be a central extension of $\operatorname{PSU}_{n}\left(q^{t}\right)$, excluding the groups listed above the proposition, and let $M$ denote a non-Weil simple module. We first con- 
sider the case $n=3$, where $\operatorname{dim}(M) \geq \frac{1}{6}\left(q^{t}-1\right)\left(q^{2 t}+3 q^{t}+1\right)$, and $p$ is either 2 or 3 , or divides one of $q^{t}-1, q^{t}+1$, or $q^{2 t}-q^{t}+1=\Phi_{6}\left(q^{t}\right)$. We apply Lemma 25 to replace $\Phi_{d}\left(q^{t}\right)$ by $t \Phi_{d}(q)$, as in the case for $\operatorname{PSL}_{n}\left(q^{t}\right)$. Unless $q^{t}=3$ and $p=2$, from [10, Theorem 4.1] we have that $\alpha(u) \leq 3$.

If $p \neq 2,3$, we have that $o(u) \leq t^{2} \Phi_{d}(q)$, with the Weyl contribution being trivial, the toral contribution being $t \Phi_{d}(q)$ for $d=1,2,6$, and the outer contribution being $t$. (Note that diagonal automorphisms need not be considered, as in $\operatorname{PSL}_{n}\left(q^{t}\right)$, using Proposition 26.)

If $d=1,2$, then we only end up with $\operatorname{PSU}_{3}(3)$, and if $d=6$, then since this is a regular number $C_{G_{0}}(u)$ is abelian by Lemma 27, and hence if $M$ is minimally active, then $\operatorname{dim}(M) \leq 2 o(u)$ by Lemma 8 . This forces $t=1$ and $q<7$, so only $q=3,5$, since $q=2,4$ are excluded. For $q=3,5, q^{2}-q+1=7,21$, and so $p=7$ in both cases. However, this now excludes $q=5$, leaving only $q=3$. Here, if $M$ is not a Weil module, then $\operatorname{dim}(M) \geq 14$, and there are therefore no examples.

If $p=3$, then the toral contribution is at most $q+1$, the Weyl contribution is 3 and the outer contribution is $t$, so we place this in our formula to get only $(n, q, t)=(3,2,3),(3,5,1)$. The exponents of the Sylow 3 -subgroups of the automorphism group $\operatorname{Aut}\left(\mathrm{PSU}_{3}\left(q^{t}\right)\right)$ for $q^{t}=5,8$ are 3 and 9, respectively, whereas $\operatorname{dim}(M) \geq 20,56$, respectively, from [16]. Thus there are no minimally active modules for $p=3$.

Suppose that $p=2$. The toral contribution is at most $t(q+1)$, the Weyl contribution is at most $n$, and the outer contribution is at most $2 t$. Unless $n=4$ or $G_{0}=\operatorname{PSU}_{3}(3)$, we have $\alpha(u) \leq n$. Thus $\alpha(u) \cdot o(u) \leq 2 t^{2} n^{2}(q+1)$, and for this to be at least $\operatorname{dim}(M)$ (for any non-trivial $M$, not just non-Weil modules), for $n \geq 5$ we have that $(n, q, t)=(5,3,1),(6,3,1)$. In these two cases, $\operatorname{dim}(M) \geq 60,182$, whereas the exponent of the Sylow 2-subgroup of $\operatorname{Aut}\left(\mathrm{PSU}_{5}(3)\right)$ is 16 , and the exponent of the Sylow 2-subgroup of $\mathrm{PSU}_{6}(3)$ is 16, so that of the automorphism group is at most 32. If $v \in \mathrm{PSU}_{n}$ (3) for $n=5,6$ has order 8 or 16 , then $\alpha(v) \leq 3$, so that $M$ cannot be minimally active.

If $n=4$, then $\alpha(u) \leq 6$, and this yields $q=3,5,7$ for $t=1$, and $q=3$ for $t=2$. For $q=3,5,7,9, \operatorname{dim}(M) \geq 20,104,300,656$, with the exponents of the Sylow 2-subgroups of $G_{0}$ being $8,8,16,16$, respectively. Thus only $q=3$ can yield a minimally active module, but $\mathrm{PSU}_{4}(3)$ is excluded from consideration.

For $n=3$ we need better bounds, because for $t=1$ we get $q \leq 19$ satisfying the bound

$$
2 t^{2} n^{2}(q+1) \geq \frac{q^{n t}-q^{t}}{q^{t}+1},
$$

and for $t=2$ we get $q=3$. The Weyl contribution ( $n$ in the above inequality) may be replaced by 2 , and the toral contribution $(t(q+1)$ above $)$ may be replaced by 
the 2-part of $\frac{1}{2}\left(q^{2 t}-1\right)$. Doing so yields $q^{t} \leq 9$, and replacing $o(u)$ with the correct exponents, which are $8,8,16,16$ for $q^{t}=3,5,7,9$, respectively, means that $q^{t}=9$ can be excluded, as $\operatorname{dim}(M) \geq 6,20,42,72$. If $\alpha(u)=2$ for $u$ of maximal order, then this will exclude $q^{t}=5,7$ as well: this can be checked and is indeed the case, yielding $G_{0}=\operatorname{PSU}_{3}(3)=G_{2}(2)^{\prime}$, so already considered. This completes the proof for $p=2$, all simple modules and all $n \geq 3$.

We now let $n=4$, and now $p$ is odd. If $p>3$, then the Sylow $p$-subgroup is abelian, so $d=1,2,4,6$, and the toral contribution is at most $t\left(q^{2}+1\right)$; the Weyl contribution is 1 ; and the outer contribution is $t$. Since $\alpha(u) \leq n$ and $\operatorname{dim}(M) \geq$ $\frac{1}{2}\left(q^{2 t}+1\right)\left(q^{2 t}-q^{t}+1\right)-1$, plugged into $\alpha(u) \cdot o(u)>\operatorname{dim}(M)$ yields

$$
n t^{2}\left(q^{2}+1\right)>\frac{1}{2}\left(q^{2 t}+1\right)\left(q^{2 t}-q^{t}+1\right)-1,
$$

which yields $t=1$ and $q=2,3$, both of which are excluded from consideration.

If $p=3$, then the Weyl contribution is 3 and the toral contribution is at most $t(q+1)$, with $t$ the outer contribution, yielding

$$
3 n t^{2}(q+1)>\frac{1}{2}\left(q^{2 t}+1\right)\left(q^{2 t}-q^{t}+1\right)-1 .
$$

Again, only $q^{t}=2,3$ satisfy this, which have been excluded.

Thus $n \geq 5$. First suppose that the Sylow $p$-subgroup is abelian. The toral contribution is at most $t\left(q^{n}-1\right) /(q-1)$ (as this is greater than $t\left(q^{n}+1\right) /(q+1)$, and the order $d$ of $q^{t}$ modulo $p$ is either at most $n$ or $2 m$ for some odd $\left.m \leq n\right)$, the Weyl contribution is 1 , and the outer contribution is at most $t$. For $M$ a non-Weil module, the inequality $\alpha(u) \cdot o(u)<\operatorname{dim}(M)$ becomes

$$
\frac{n t^{2}\left(q^{n}-1\right)}{q-1}>\frac{q^{(n-2) t}\left(q^{t}-1\right)\left(q^{(n-2) t}-q^{t}\right)}{q^{t}+1},
$$

and this forces $t=1$ and $(n, q)=(5,2),(5,3),(6,2),(7,2),(8,2)$. To eliminate these, we first ignore the $t \mathrm{~s}$, and then produce better estimates for the toral contribution than $\left(q^{n}-1\right) /(q-1)$ : for $n=5,7$ we get $\left(q^{n}+1\right) /(q+1)$, which eliminates $(5,3)$ and $(7,2)$, and for $n=8$ we use $\left(q^{7}+1\right) /(q+1)$, which eliminates $(8,3)$. Since $(6,2)$ is not being considered in this proposition, we are left with $\operatorname{PSU}_{5}(2)$. Here $p=5,11, \operatorname{dim}(M) \geq 43$ for non-Weil modules from [16, pp. 182-184], and it is easy to see that $\alpha(u)=2$ for $o(u)=5,11$ by a computer check (alternatively we can use the fact that $C_{G}(u)$ is abelian and apply Lemma 8$)$. Thus there are no minimally active non-Weil modules in this case.

If the Sylow $p$-subgroup is non-abelian, then, as with the linear case, the toral contribution is at most $t\left(q^{\lfloor n / 3\rfloor}+1\right)$ and the Weyl contribution is at most $n$, yielding

$$
n^{2} t^{2}\left(q^{\lfloor n / 3\rfloor}+1\right)>\frac{q^{(n-2) t}\left(q^{t}-1\right)\left(q^{(n-2) t}-q^{t}\right)}{q^{t}+1},
$$


where we get $t=1$ and $(n, q)=(5,2),(6,2)$, although $\mathrm{PSU}_{6}(2)$ is excluded. For $G_{0}=\mathrm{PSU}_{5}$ (2), only the Sylow 3-subgroup (and the Sylow 2-subgroup of course) is non-abelian, and $\operatorname{Out}\left(G_{0}\right)$ has order 2 , we have $G_{0}=G$ and so $u$ has order at most 9 , and $\alpha(u)=2$ if $o(u)=9$, and $\alpha(u) \leq 5$ if $o(u)=3$, with $\operatorname{dim}(M) \geq 44$ from [16, p. 181]. Thus there is no non-Weil simple minimally active module for this group.

As with linear groups, we now check that if $u$ induces an automorphism that is not inner-diagonal on $G_{0}$, then $u$ does not act minimally actively on a Weil module. From the previous proposition we may assume that $p$ is odd, so that $t \geq 3$.

Suppose that the Sylow $p$-subgroup of $G_{0}$ is abelian: as in the proof of the proposition we see that $o(u) \leq t^{2}\left(q^{n}-1\right) /(q-1)$, and so we get

$$
\frac{n t^{2}\left(q^{n}-1\right)}{q-1}>\frac{q^{n t}-q^{t}}{q^{t}+1}
$$

yielding $(n, q)=(3,2),(4,2)$ for $t=3$, and no solutions for $t \geq 5$. Thus $p=3$, but the Sylow 3-subgroup of $G_{0}$ is definitely not abelian.

If the Sylow $p$-subgroup of $G_{0}$ is non-abelian, then the toral contribution is at most $t\left(q^{\lfloor n / 3\rfloor}+1\right)$, the Weyl contribution is at most $n$, and this time we get

$$
n^{2} t^{2}\left(q^{\lfloor n / 3\rfloor}+1\right)>\frac{q^{n t}-q^{t}}{q^{t}+1}
$$

and this yields $(n, q, t)=(3,2,3)$ as the only solution, so again $p=3$. The Sylow 3 -subgroup of Aut $\left(\mathrm{PSU}_{3}(8)\right)$ has exponent 9, and the dimension of a Weil module is 56 , with $\alpha(u) \leq 3$ by [10, Theorem 4.1 , so $u$ cannot act minimally actively on a Weil module by Lemma 5 (iv).

This completes the proof of Proposition 29 for unitary groups.

\subsection{Groups $\operatorname{Sp}_{2 n}(q)$}

For this subsection we let $G_{0}$ be a quotient of $\operatorname{Sp}_{2 n}\left(q^{t}\right)$, and we exclude the cases of $\mathrm{Sp}_{4}(2)=\mathrm{Sym}_{6}, \mathrm{PSp}_{4}(3)=\mathrm{PSU}_{4}(2)$ and $\mathrm{Sp}_{6}(2)$ (the last two appear in Proposition 24). Suppose that $M$ is a non-trivial simple module, but not a Weil module, which exist only for odd $q$. From the table near the start of this section, the dimension of $M$ is at least $q^{t}\left(q^{n t}-1\right)\left(q^{(n-1) t}-1\right) / 2\left(q^{t}+1\right)$.

Proposition 32. Suppose that $G_{0}$ is a central extension of a symplectic group $\operatorname{PSp}_{2 n}\left(q^{t}\right)$ for some $n \geq 2$, with $\left(n, q^{t}\right) \neq(4,2),(4,3),(6,2)$, and let $u$ be a p-element of $G$ such that $G=\left\langle G_{0}, u\right\rangle$. If $u$ acts minimally actively on a non-trivial simple module $M$ that is not a Weil module, then $G=\operatorname{Sp}_{4}(4), p=17, o(u)=17$, and $\operatorname{dim}(M)=18$. 
Proof. Let $G_{0}=\operatorname{Sp}_{2 n}\left(q^{t}\right)$ for some $n \geq 2$, some prime power $q$ and some $t \geq 1$. As in previous sections, $u$ induces an automorphism that projects $\operatorname{in} \operatorname{Out}\left(G_{0}\right)$ to a field automorphism of order $t$. Since $M$ is not a Weil module, we have

$$
\operatorname{dim}(M) \geq \frac{\left(q^{n t}-1\right)\left(q^{n t}-q^{t}\right)}{2\left(q^{t}+1\right)} .
$$

Let $d$ be the order of $q^{t}$ modulo $p$.

If $d=2 n$, then $u$ is regular, so $\operatorname{dim}(M)<2 o(u)$ by Lemma 27 . We have that $o(u) \leq t^{2}\left(q^{n}+1\right)$ by Lemma 25 , since the outer contribution is $t$ and the toral contribution is at most $t\left(q^{n}+1\right)$, so we get

$$
2 t^{2}\left(q^{n}+1\right) \geq \frac{\left(q^{n t}-1\right)\left(q^{n t}-q^{t}\right)}{2\left(q^{t}+1\right)} .
$$

If $t=1$, then the solutions to this are $q \leq 5$ for $n=2$, and $q=2$ for $n=3$. If $t>1$, we only get $(n, q, t)=(2,2,2)$, but this needs $p \mid t=2$ and $p \nmid q=4$, a contradiction. We of course exclude $(n, q)=(2,2),(2,3),(3,2)$ for $t=1$, as we stated above, so we are left with $(n, q)=(2,4),(2,5)$ for $t=1$. Here $d=4$, and $\Phi_{d}(q)=q^{2}+1: 4^{2}+1=17$ and $5^{2}+1=26$. For $q=5$ this means that $o(u)=13$, and $\operatorname{dim}(M) \geq 40$, so this cannot work, but for $\operatorname{Sp}_{4}(4)$, the module of dimension 18 could be minimally active. Since $C_{G}(u)=\langle u\rangle$ in this case, it is minimally active by Lemma 8.

Suppose that the Sylow $p$-subgroup is abelian. If $d \neq n$, then we have the bound $o(u) \leq t^{2}\left(q^{d}+1\right)$ with $d \leq n-1$, and note that $n \geq 3$. We also have that $\alpha(u) \leq n+3$ by [10, Theorem 4.3], so using Lemma 5 (iv) we get

$$
(n+3) \cdot t^{2}\left(q^{d}+1\right) \geq \alpha(u) o(u) \geq \operatorname{dim}(M) \geq \frac{\left(q^{n t}-1\right)\left(q^{n t}-q^{t}\right)}{2\left(q^{t}+1\right)},
$$

and the only solutions are for $t=1$, with $(n, q)=(3,2),(4,2)$. The first can be ignored using Proposition 24, and for the second we have $d=1,2,3,4,6$, which yield $\Phi_{d}(2)=1,3,7,5,3$. Since the Sylow 3-subgroup of $\operatorname{Sp}_{8}(2)$ is non-abelian, we only get the cases $d=3,4$, so $p=7,5$. For $p=7$ it is easy to check with a computer that $\alpha(u)=2, o(u)=7$, and $\operatorname{dim}(M) \geq 35$ by the degree bound above. Thus there is no (non-trivial) minimally active simple module for this group. For $p=5$ we have $o(u)=5$ and $\alpha(u) \leq 3$, so that again there are no examples.

We need also consider the case where $d=n$, so that $o(u) \leq t^{2}\left(q^{n}-1\right) /(q-1)$. Using $\alpha(u) \leq(n+3)$ and Lemma 5 (iv), we get that if $M$ is minimally active, then

$$
\frac{(n+3) \cdot t^{2}\left(q^{n}-1\right)}{q-1} \geq \alpha(u) o(u)>\operatorname{dim}(M) \geq \frac{\left(q^{n t}-1\right)\left(q^{n t}-q^{t}\right)}{2\left(q^{t}+1\right)},
$$


which for $t=1$ yields the solutions $(n, q)=(2,2),(2,3),(2,4),(3,2),(4,2)$, $(5,2)$, and for $t \geq 2$ yields only the solution $(n, q)=(2,2)$ for $t=2$, which we noticed earlier is not an example because $p \mid t=2$ and $p \nmid q=4$. If $n$ is odd, then $n$ is a regular number, so we may replace $n+3$ by 2 , as in the previous case, and this removes the case $(5,2)$. The cases $(2,2),(2,3),(3,2)$ are excluded from our analysis, and $(4,2)$ has been dealt with, leaving only $\operatorname{Sp}_{4}(4)$, with $p \mid \Phi_{2}(4)=5$. We have $\operatorname{dim}(M) \geq 18, o(u)=5$ and $\alpha(u) \leq 3$ by a computer calculation, so there is no example here either.

We may therefore assume that the Sylow $p$-subgroup is non-abelian, and hence $p$ divides the order of the Weyl group of type $C$, which is $Z_{2} 2 \operatorname{Sym}_{n}$, and $p \leq n$ with $p$ dividing two separate tori, so that $p \mid \Phi_{d}(q)$ and $p \mid \Phi_{p d}(q)$. If $p$ is odd, then in particular this means that $d \leq \frac{n}{3}$ or $d$ is even and $d \leq \frac{2 n}{3}$, so in either case the toral contribution is at most $t\left(q^{d}+1\right)$. The Weyl contribution is at most $n$, and the outer contribution is $t$, so $o(u) \leq n t^{2}\left(q^{d}+1\right)$. This is of course maximized at $d=\left\lfloor\frac{n}{3}\right\rfloor$. As $\alpha(u)$ is still at most $n+3$, we get

$$
(n+3) \cdot n t^{2}\left(q^{\lfloor n / 3\rfloor}+1\right) \geq \alpha(u) o(u)>\operatorname{dim}(M) \geq \frac{\left(q^{n t}-1\right)\left(q^{n t}-q^{t}\right)}{2\left(q^{t}+1\right)},
$$

The only solution is $(n, q, t)=(3,2,1)$, which we have already excluded.

Thus $p=2$. Here there is a diagonal automorphism, but we do not need to consider these by Proposition 26, so the outer contribution is $t$. The Weyl contribution is $2 n$, and the toral contribution is at most $t(q+1)$ by Lemma 25 , so the order of a 2-element of $G_{0}$ is at most $2 n t^{2}(q+1)$ by Proposition 26 . Since $\alpha(u) \leq 2 n$, this yields $\alpha(u) \cdot(o(u)-1) \leq 2 n\left(2 n t^{2}(q+1)-1\right)$. If $M$ is minimally active, then, by Lemma 5 (iv)

$$
2 n\left(2 n t^{2}(q+1)-1\right) \geq \alpha(u)(o(u)-1) \geq \operatorname{dim}(M) \geq \frac{\left(q^{n t}-1\right)\left(q^{n t}-q^{t}\right)}{2\left(q^{t}+1\right)},
$$

which has no solutions for $t \neq 1$, and for $t=1$ we get $(n, q)=(2,2),(2,3)$, $(2,5),(3,2)$, with the first and last eliminated since $q$ must be odd. Since $\operatorname{Sp}_{4}(3)$ is also excluded, this leaves $G_{0}=\mathrm{Sp}_{4}(5)$ : from [2, p.63] we see that $o(u) \leq 8$, and $\alpha(u) \leq 4$ with $\operatorname{dim}(M) \geq 40$, so $M$ cannot be minimally active. This completes the proof.

We now have to complete the proof of Proposition 29 by checking that if $u$ induces an outer automorphism on $G_{0}=\operatorname{Sp}_{2 n}\left(q^{t}\right)$, then $u$ cannot act minimally actively on a Weil module. First suppose that $p$ is odd, so that $u$ induces a field automorphism and $t \geq 3$.

The dimension of $M$ is $\frac{1}{2}\left(q^{n t} \pm 1\right)$ (recall that $q$ must be odd) and as we saw above, if the Sylow $p$-subgroup of $G_{0}$ is abelian, then $o(u) \leq t^{2}\left(q^{n}+1\right)$, and 
as $\alpha(u) \leq n+3$, if $u$ acts minimally actively, then by Lemma 5 (iv) we have

$$
t^{2}(n+3)\left(q^{n}+1\right) \geq \frac{1}{2}\left(q^{n t}-1\right),
$$

which yields $(n, q, t)=(2,3,3)$, but of course $t$, which is a power of $p$, cannot divide $q$, so we get no examples.

If the Sylow $p$-subgroup is non-abelian, then

$$
\alpha(u) o(u) \leq(n+3) n t^{2}\left(q^{\lfloor n / 3\rfloor}+1\right),
$$

as we saw in the proof of the previous proposition: thus we have

$$
(n+3) n t^{2}\left(q^{\lfloor n / 3\rfloor}+1\right) \geq \frac{1}{2}\left(q^{n t}-1\right),
$$

and this has no solutions.

We thus reduce to $p=2$. In this case, from [9, Section 5], we see that there are two Weil modules, which have dimension $\frac{1}{2}\left(q^{n}-1\right)$ and are swapped by the diagonal automorphism. By Theorem 28, $v \in G_{0}$ cannot act on these Weil modules with a single Jordan block, and hence by Lemma 9 if $u$ induces a diagonal automorphism on $G_{0}$, then it cannot act minimally actively on the sum of the two Weil modules.

Thus $u$ acts as either a field automorphism or the product of a field and diagonal (whichever stabilizes the two Weil modules), but in either case $t \geq 2$.

We have already bounded $o(u)$ by $2 n t^{2}(q+1)$, so with $\alpha(u) \leq 2 n$ we get

$$
2 n\left(2 n t^{2}(q+1)-1\right) \geq \alpha(u) \cdot(o(u)-1) \geq \operatorname{dim}(M) \geq \frac{1}{2}\left(q^{n t}-1\right) .
$$

If $t \geq 4$, then there are no solutions, and for $t=2$ we get solutions $(n, q)=(2,3)$, $(2,5),(3,3)$. The exponents of the Sylow 2-subgroups of $\mathrm{PSp}_{4}(9), \mathrm{PSp}_{4}(25)$ and $\mathrm{PSp}_{6}(9)$ are $8,8,16$, respectively, so $o(u) \leq 16,16,32$, respectively. The dimensions of the Weil modules are 40, 312, 364, respectively, and $\alpha(u) \leq 4,4,6$, respectively, so the formula $\operatorname{dim}(M)<\alpha(u) \cdot o(u)$ eliminates the second and third options from being minimally active. Finally, for $\mathrm{PSp}_{4}(9)$, if we can reduce $\alpha(u)$ for $u$ of order 16 (hence $v=u^{2} \in G_{0}$ of order 8) to 2, then we are done: this is the case by an easy computer calculation, and we complete the proof of Proposition 29.

\subsection{Groups $\Omega_{2 n+1}(q)$ and $\Omega_{2 n}^{ \pm}(q)$}

As we saw in Table 4, the minimal degree for $\operatorname{Spin}_{2 n+1}(q)$ for $(n, q) \neq(3,3)$ is $q^{n-1}\left(q^{n-1}-1\right)$.

Recall that the polynomial order of $\operatorname{Spin}_{2 n+1}(q)$ is $q^{n^{2}} \prod_{i=1}^{n}\left(q^{2 i}-1\right)$, so that if $p \nmid q$ divides the order of $\operatorname{Spin}_{2 n+1}(q), p$ divides $q^{d} \pm 1$ for some $1 \leq d \leq n$. 
Proposition 33. Let $G_{0}$ be a central extension of one of the groups $\Omega_{2 n+1}(q)$ for $(n, q) \neq(3,3)$ and $n \geq 3$. Let $u$ be a p-element of $G$ such that $G=\left\langle G_{0}, u\right\rangle$. There are no non-trivial minimally active simple modules for $G$.

Proof. Let $G_{0}=\Omega_{2 n+1}\left(q^{t}\right)$ for some $n \geq 3$, some prime power $q$, and some $t \geq 1$, and suppose that the Sylow $p$-subgroup of $G_{0}$ is abelian, so that $p$ divides a single cyclotomic polynomial, and let $d$ be the order of $q^{t}$ modulo $p$. The order of $u$ is at most $t \cdot t \Phi_{d}(q) \leq t^{2} \cdot\left(q^{n}+1\right)$. As $\alpha(u) \leq n+3$ by [10, Theorem 4.4], we get using Lemma 5 (iv)

$$
(n+3) \cdot t^{2}\left(q^{n}+1\right)>\alpha(u) \cdot o(u)>q^{t(n-1)}\left(q^{t(n-1)}-1\right)
$$

if $M$ is minimally active, and this forces $t=1$ and $(n, q)=(3,3),(3,5)$. Omitting the $t$ from now on, replacing the upper bound $q^{n}+1$ for $\Phi_{d}(q)$ by each of $\left(q^{n}+1\right) /(q+1),\left(q^{n}-1\right) /(q-1),(q-1)$ and $(q+1)$ eliminates $(n, q)=(3,5)$, and $(n, q)=(3,3)$ is excluded already, so there are no solutions.

We now may assume that the Sylow $p$-subgroup is non-abelian, so that if $p$ is odd then $o(u) \leq t \cdot n \cdot t\left(q^{d}+1\right)$ (as the Weyl group of type $B$ is the Weyl group of type $C$ we can use the Weyl contribution from Proposition 32), but with both $d$ and $p d$ dividing $2 n$. We thus get

$$
(n+3) \cdot n \cdot t^{2}\left(q^{d}+1\right)>\alpha(u) \cdot o(u)>q^{t(n-1)}\left(q^{t(n-1)}-1\right),
$$

for $d \leq \frac{n}{3}$, which is obviously maximized at $d=\lfloor n / 3\rfloor$, still with no solutions.

If $p=2$, then we get $o(u) \leq 2 t \cdot 2 n \cdot t(q+1)$ using Proposition 26 and the fact that the order of an outer automorphism is at most $2 t$, and so now we have

$$
2 n \cdot 4 n t^{2}(q+1)>\alpha(u) \cdot o(u)>q^{t(n-1)}\left(q^{t(n-1)}-1\right),
$$

(as $\alpha(u) \leq 2 n$ this time) which again has no solutions for $(n, q, t) \neq(3,3,1)$. This completes the proof.

Having dispensed with the odd-dimensional orthogonal groups, we turn to the even-dimensional ones. For $\Omega_{2 n}^{+}(q)$, the minimal degree is $q^{n-2}\left(q^{n-1}-1\right)$ (unless $\left.G_{0}=\Omega_{8}^{+}(2)\right)$ and for $\Omega_{2 n}^{-}(q)$ the minimal degree is $\left(q^{n-2}-1\right)\left(q^{n-1}+1\right)$, so in both cases $\operatorname{dim}(M)>\left(q^{n-1}-1\right)\left(q^{n-2}-1\right)$. If we use this bound, then we can deal with both cases simultaneously. The polynomial order of $\operatorname{Spin}_{2 n}^{ \pm}(q)$ is

$$
q^{n(n-1)}\left(q^{n} \mp 1\right) \prod_{i=1}^{n-1}\left(q^{2 i}-1\right) .
$$

We already found minimally active modules for $2 \cdot \Omega_{8}^{+}(2)$ in Proposition 24 , and the next proposition says that there are no more. 
Proposition 34. Let $G_{0}$ be a central extension of one of the groups $\Omega_{2 n}^{ \pm}(q)$ other than $\Omega_{8}^{+}(2)$, for $n \geq 4$. Let $u$ be a p-element of $G$ such that $G=\left\langle G_{0}, u\right\rangle$. There are no non-trivial minimally active simple modules for $G$.

Proof. Our proof works the same as Proposition 33. If the Sylow $p$-subgroup is abelian, then $p$ is odd and $o(u) \leq t^{2} \Phi_{d}(q) \leq t^{2}\left(q^{n}+1\right)$. Placing this in our standard formula from Lemma 5 (iv), using $\alpha(u) \leq n+3$ from [10, Theorem 4.4] gives

$$
(n+3) t^{2}\left(q^{n}+1\right) \geq \operatorname{dim}(M)>\left(q^{t(n-1)}-1\right)\left(q^{t(n-2)}-1\right) .
$$

This bound yields no solutions for $t \geq 3$, and for $t=1$ the solutions

$$
(n, q)=(4,2),(4,3),(4,4),(4,5),(4,7),(5,2),(6,2) .
$$

If $q$ is odd, then we may replace $\left(q^{n}+1\right)$ by $\frac{1}{2}\left(q^{n}+1\right)$, removing $(4,5)$ and $(4,7)$ from the list.

If $d=n$ or $d=2 n$, then $u$ is regular, so $C_{G_{0}}(u)$ is abelian by Lemma 27, and so in this case we may replace $\alpha(u) \cdot(o(u)-1)$ by $2 o(u)$ via Lemma 8 , and so (removing the $t$, which is equal to 1 anyway)

$$
2\left(q^{n}+1\right)>\left(q^{n-1}-1\right)\left(q^{n-2}-1\right),
$$

which only has a solution for $(n, q)=(4,2)$. Thus we may assume that $d \neq n, 2 n$, in which case we may replace $o(u)$ by $t^{2}\left(q^{n-1}+1\right)$. Using this we reduce our possibilities to $(4,2)$ and $(5,2)$.

As $\Omega_{8}^{+}(2)$ is excluded, we just consider $G_{0}=\Omega_{8}^{-}(2)$ : from [16] we see that $\operatorname{dim}(M) \geq 33$, and $o(u)=3,5,7,9,17$. Furthermore, we have that $\alpha(u)=2$ for $o(u)>3$, and $\alpha(u) \leq 4$ for $o(u)=3$, so there are no examples using the formula $\operatorname{dim}(M) \leq \alpha(u) \cdot(o(u)-1)$.

For $G_{0}$ a central extension of $\Omega_{10}^{ \pm}(2), \operatorname{dim}(M)$ is at least the smallest of $2^{5-2}\left(2^{5-1}-1\right)=120$ and $\left(2^{5-1}+1\right)\left(2^{5-2}-1\right)=119$, so $\operatorname{dim}(M) \geq 119$. For $\Omega_{10}^{+}(2), o(u) \in\{3,5,7,9,17,31\}$, and for $o(u) \geq 7$ we have that $\alpha(u)=2$, with $\alpha(u) \leq 3$ for $o(u)=5$ and $\alpha(u) \leq 5$ for $o(u)=3$, which shows that $u$ cannot act minimally actively on a non-trivial $M$. For $\Omega_{10}^{-}(2), o(u) \in\{3,5,7,9,11,17\}$, and the same statements hold for $\alpha(u)$, so again $u$ cannot act minimally actively on a non-trivial $M$.

Suppose that $p$ is still odd, but that the Sylow $p$-subgroup is non-abelian. Thus $p$ divides both $\Phi_{d}\left(q^{t}\right)$ and $\Phi_{d p}\left(q^{t}\right)$, and $d \leq \frac{n}{3}$. Since the Weyl group of type $D$ is a subgroup of the Weyl group of type $B$, we see that the exponent of the Sylow $p$-subgroup of the Weyl group is at most $n$. Thus $o(u) \leq t^{2} \cdot n \cdot\left(q^{\lfloor n / 3\rfloor}+1\right)$ and $\alpha(u) \leq n+3$, and thus we need to check

$$
t^{2} n(n+3)\left(q^{\lfloor n / 3\rfloor}+1\right) \geq\left(q^{t(n-1)}-1\right)\left(q^{t(n-2)}-1\right),
$$


which only has solutions for $t=1$, and then $(n, q)=(4,2),(5,2)$, which we have already checked. This completes the proof for $p$ odd.

Suppose that $p=2$, so that the order of $u$ is at most $4 t^{2} \cdot 2 n \cdot(q+1)$ : $\operatorname{Out}\left(G_{0}\right)$ has exponent at most $4 t$, the Weyl contribution is at most $2 n$, and the toral contribution is at most $t(q+1)$. (To see that the exponent of Sylow 2-subgroup of $\operatorname{Out}\left(G_{0}\right)$ is at most $4 t$ and not $8 t$, note that if $n$ is even, then the diagonal automorphisms form $Z_{2} \times Z_{2}$, so we are done, and if $n$ is odd, then the diagonal automorphisms form either $Z_{2}$ or $Z_{4}$, with the graph automorphism inverting this group [7, Theorem 2.5.12 (i)].) Since $\alpha(u) \leq 2 n$, we get

$$
16 t^{2} n^{2}(q+1) \geq\left(q^{t(n-1)}-1\right)\left(q^{t(n-2)}-1\right),
$$

which has a solution only for $(n, q, t)=(4,3,1)$. However, although information on the Sylow 2-subgroups of $\operatorname{Aut}\left(\Omega_{8}^{ \pm}(3)\right)$ is not available in [2], there are constructions of them on the online Atlas, and hence a computer algebra package immediately tells you that the exponent is 8 , not 32 as suggested by the formula above. This proves that there are no minimally active modules for $p=2$.

\subsection{Groups $\mathrm{SL}_{2}(q)$}

This short subsection deals with the groups $G_{0}=\mathrm{SL}_{2}(q)$, where $p \nmid q, q \geq 4$ and $q \neq 4,5,7,9$ (as these are alternating groups or are given in Proposition 24). In [5, Theorem 1.2], if $\operatorname{SL}_{2}(q) \leq G \leq \mathrm{GL}_{2}(q)$, then all possibilities for $u$ acting minimally actively are determined, and given by the following lemma.

Lemma 35. Let $G_{0}$ be a central extension of $\operatorname{PSL}_{2}(q)$ for $q \neq 4,5,7$, 9. Suppose that $u$ induces an inner-diagonal automorphism on $G_{0}$. If $u$ acts minimally actively on $M$, then one of the following holds:

(i) $G=\mathrm{SL}_{2}(q)$ for $q=2^{a}, p=2^{a} \pm 1$ is a Fermat or Mersenne prime, $o(u)=p, M$ is any simple module,

(ii) $G=\mathrm{SL}_{2}(q)$ for $q$ odd, $p$ is odd, $\frac{1}{2}(q \pm 1)=p^{a}, o(u)=p^{a}, \operatorname{dim}(M) \leq$ $o(u)+1$,

(iii) $G=\mathrm{PSL}_{2}(q)$ or $\mathrm{PGL}_{2}(q), q$ is a Fermat or Mersenne prime, $p=2$, and $\operatorname{dim}(M) \leq o(u)+1$.

Thus we can assume that $u \in G$ acts as a field or product of a field and diagonal automorphism. Let $G_{0}$ be a central extension of $\operatorname{PSL}_{2}\left(q^{t}\right)$ for some prime power $q$ and some $t \geq 2$ a power of $p$, with $G=\left\langle G_{0}, u\right\rangle$ and $\left|G: G_{0}\right|=t$. Note that $\frac{1}{2}\left(q^{t}-1\right)$ is the smallest dimension of a non-trivial simple module for $G_{0}$ if $q$ is odd, and $q^{t}-1$ is the smallest dimension if $q$ is even. 
Suppose that $\langle u\rangle \cap G_{0}=1$. If $o(u)$ is even, then $\alpha(u) \leq 4$ by [10, Lemma 3.1], so $\operatorname{dim}(M) \leq 4(t-1)$ by Lemma 5 (iv). This yields $\frac{1}{2}\left(q^{t}-1\right) \leq 4(t-1)$, so $t=2$ and $q^{t} \leq 9$. Having excluded 4, 5, 7, 9, and since 8 need not be considered, there are no solutions.

If $o(u)$ is odd, then as $\alpha(u)=2$ by [10, Lemma 3.1], we see that $\operatorname{dim}(M) \leq$ $2(t-1)$, and $\operatorname{dim}(M) \geq \frac{1}{2}\left(q^{t}-1\right)$. As $t \geq 3$, we only get $q^{t}=8$, but then the minimal degree is $q^{t}-1$, not $\frac{1}{2}\left(q^{t}-1\right)$, and so there are no solutions here either.

Thus $u^{t} \neq 1$, and so $p \mid(q \pm 1)$. If $p=2$, the order of $u$ is at most $t^{2}(q+1)$, and $\alpha(u)=3$ by [10, Lemma 3.1], so if $u$ acts minimally actively on $M$, then we have $\operatorname{dim}(M)<\frac{3}{2} t^{2}(q+1)$, whereas $\operatorname{dim}(M) \geq \frac{1}{2}\left(q^{t}-1\right)$. For $t=2$ this yields $q \leq 11$, for $t=4$ this yields $q=3$, and there are no solutions for $t \geq 8$.

Replacing $t(q+1)$ with the 2-part of $\frac{1}{4}\left(q^{2 t}-1\right)$ (which is the exponent of the Sylow 2-subgroup of $G_{0}$ ) yields $q \leq 9$. Finally, in the remaining cases, one may check that the exponent of the Sylow 2-subgroup of $\operatorname{Aut}\left(\operatorname{PSL}_{2}\left(q^{t}\right)\right)$ is 8 , 16 and 16 , for $q^{t}=25,49,81$, respectively, and $\operatorname{dim}(M) \geq 12,24,40$, respectively. This eliminates the case where $q^{t}=81$. Finally, in the remaining cases if $\operatorname{dim}(M) \geq q^{t}-1$, then it cannot be minimally active, so it is only the two modules of dimension $\frac{1}{2}\left(q^{t}-1\right)$ that are important: for these, a computer calculation shows that any element $v$ of $G_{0}$ acts on them with only blocks of size $o(v)$, whence $u$ cannot act minimally actively.

Thus $p$ is odd. First assume that $q$ is even. The order of $p$ is at most $\frac{1}{2} t^{2}(q+1)$, and $\alpha(u)=2$ by [10, Lemma 3.1], so that if $u$ acts minimally actively, then we have $\operatorname{dim}(M) \leq t^{2}(q+1)$, whereas $\operatorname{dim}(M) \geq q^{t}-1$. The only solution to this is $q=2$ and $t=p=3$, which is the small Ree group ${ }^{2} G_{2}(3)$, hence will not be considered as it is defining characteristic.

Hence we may assume that $q$ is odd, in which case $\operatorname{dim}(M) \geq \frac{1}{2}\left(q^{t}-1\right)$. We still have that $\alpha(u)=2$ and $o(u) \leq \frac{1}{2} t^{2}(q+1) / 2$. This yields only one solution again, namely $q=3$ and $t=3$, but then this is defining characteristic and not in consideration. Thus there are no solutions when $u$ does not induce an innerdiagonal automorphism.

\section{Exceptional groups in cross characteristic}

In this section we deal with $G_{0}$ a central extension of an exceptional group of Lie type. We start by dealing with a few small groups, which feature because they have exceptional Schur multipliers and so can have unusually small minimal faithful degrees, the analogue of Proposition 24.

Proposition 36. Let $G_{0}$ be a central extension of one of the simple groups $G_{2}(3)$, $G_{2}(4), F_{4}(2),{ }^{2} B_{2}(8)$. Let $u$ be a p-element of $G$ such that $G=\left\langle G_{0}, u\right\rangle$, and 
let $M$ be a non-trivial simple module on which $u$ acts minimally actively. One of the following holds:

(i) $G=G_{2}(3), p=13, o(u)=13$ and $\operatorname{dim}(M)=14$,

(ii) $G=2 \cdot G_{2}(4), p=13, o(u)=13$ and $\operatorname{dim}(M)=12$,

(iii) $G=2 \cdot{ }^{2} B_{2}(8), p=13, o(u)=13$ and $\operatorname{dim}(M)=14$ (two representations).

The field of definition is always $\mathbb{F}_{13}$.

Proof. The group $G_{2}(3)$ has outer automorphism group of order 2 and Schur multiplier of order 3 , with the outer automorphism of $3 \cdot G_{2}(3)$ inverting the centre, so we only have to consider the groups $G=G_{2}(3), G=3 \cdot G_{2}(3)$ and $G=G_{2}(3)$.2. We simply check these one by one for $p=2,7,13$, and get the single example above.

The group $G_{2}(4)$ has outer automorphism group of order 2 and Schur multiplier of order 2 , so we need to consider $G_{2}(4)$ and $2 \cdot G_{2}(4)$ for $p$ odd (so $p=3,5,7,13)$, where the minimal degree is 12 . The order of $u$ must be $p$, and for $p=5,7,13$ two conjugates of $u$ generate $G$, so we can exclude $p=3,5$ by Lemma 5 (iv) and focus on the 12-dimensional simple module for $2 \cdot G_{2}(4)$ for $p=7,13$. As the centralizer of an element of order 7 in $G_{2}(4)$ has order 21 , we get $\operatorname{dim}(M) \leq 10$ by Lemma 8 and so can exclude this as well, leaving just $p=13$, where for $G_{2}(4)$ the centralizer has order exactly 13 , so an application of the same lemma shows that the simple module of dimension 12 is minimally active for $u$.

For the group $G=F_{4}(2)$, there is an exceptional Schur multiplier of order 2: for $G=2 \cdot F_{4}(2)$, the character degrees are known for $p=5,7,13,17$, but the full set of character degrees is not known for $p=3$. For $p \geq 5$, the minimal faithful degree is 52, and for $p=3$ (see [27]) states that it is at least 44 . A computer calculation shows that $G$ is generated by two conjugates of $u$ for $o(u)=5,7,9,13,17$, and so there are no minimally active modules for these elements. For $o(u)=3$, three conjugates suffice, and so there are no minimally active modules here either.

Now let $G_{0}$ be a central extension of the group ${ }^{2} B_{2}(8)$, where the exceptional Schur multiplier is a Klein four group, but all extensions $2 \cdot{ }^{2} B_{2}(8)$ are isomorphic because the outer automorphism of order 3 permutes them. Here $p=5,7,13$, as we can discount $p=3$, since $o(u)=3$ and $\alpha(u) \leq 3$ by [10, Proposition 5.8], and $\operatorname{dim}(M) \geq 14$. Thus we just check all simple modules for $G=2 \cdot{ }^{2} B_{2}(8)$ and $o(u)=5,7,13$, noting that $M$ is minimally active if and only if $\operatorname{dim}(M) \leq p+1$ because $C_{G_{0}}(u)=Z\left(G_{0}\right) \cdot\langle u\rangle$ via Lemma 8 . We find just the one example for $p=13$. 
In [10, Theorem 5.1], it is shown that $\alpha(G) \leq \ell+3$ if $G$ is of exceptional type, where $\ell$ is the untwisted rank of $G$ (with one exception of $G$ of type $F_{4}$ and $p=2$, where $\alpha(G) \leq 8)$.

We also need a bound on the orders of $p$-elements in an exceptional group of Lie type. Broadly speaking, by Proposition 26 if $q$ has order $d$ modulo $p$, then the order of a $p$-element is at most $q^{d}-1$ multiplied by the exponent of the Weyl group. We give a general bound now.

Proposition 37. Let $G_{0}$ be a central extension of one of $G_{2}(q), F_{4}(q), E_{6}(q)$, ${ }^{2} E_{6}(q), E_{7}(q)$ and $E_{8}(q)$ other than $G_{2}(2)$, with $p \nmid q$, and let $u$ be a p-element of $G$. The order $u$ is at most $q^{\ell+1}-1$, where $\ell$ is the untwisted rank of $G$.

Proof. Let $u$ be a $p$-element, and let $d$ be the order of $q$ modulo $p$. If $p$ does not divide the order of the Weyl group, then $p \geq 5\left(p \geq 7\right.$ for $\left.E_{8}(q)\right)$ and the Sylow $p$-subgroup of the socle of $G$ is abelian and has exponent at most $\Phi_{d}(q)$ by Proposition 26. The only outer automorphism of $G$ that $u$ can induce is a field automorphism, as diagonal and graph automorphisms have order 2 or 3 , and field automorphism have order less than $q-1$.

Thus $o(u) \leq \Phi_{d}(q) \cdot(q-1)$. All we therefore need is an upper estimate for all $\Phi_{d}(q)$ where $d$ divides one of the reflection degrees for $G$.

For $G=G_{2}$, the largest is $\Phi_{3}(q)=q^{2}+q+1$, so $o(u) \leq\left(q^{3}-1\right)$. For $F_{4}$, the largest is $\Phi_{8}(q)=q^{4}+1$, so $o(u) \leq\left(q^{4}+1\right)(q-1) \leq q^{5}-1$. For $E_{6}$ the largest is $\Phi_{9}(q)=q^{6}+q^{3}+1$, so $o(u) \leq\left(q^{6}+q^{3}+1\right)(q-1) \leq\left(q^{7}-1\right)$. For ${ }^{2} E_{6}(q)$ the largest is $\Phi_{18}(q)=q^{6}-q^{3}+1$, so again we have $o(u) \leq q^{7}-1$. For $E_{7}$ the largest is $\Phi_{7}(q)=q^{6}+q^{5}+q^{4}+q^{3}+q^{2}+q+1$, so $o(u) \leq q^{7}-1$ again. Finally, for $E_{8}$ the largest is $\Phi_{30}(q)=q^{8}+q^{7}-q^{5}-q^{4}-q^{3}+q+1$, so that $o(u) \leq \Phi_{30}(q)(q-1) \leq q^{9}-1$. Thus in all cases,

$$
o(u) \leq q^{\ell+1}-1
$$

Thus now $p$ divides the order of the Weyl group, so $p \leq 7$, and we use Proposition 26 again. The exponents of the Sylow $p$-subgroups of the Weyl groups of exceptional groups are given below.

\begin{tabular}{ll}
\hline Group & Exponents \\
\hline$G_{2}$ & 2,3 \\
$F_{4}$ & 8,3 \\
$E_{6}$ & $8,9,5$ \\
$E_{7}$ & $8,9,5,7$ \\
$E_{8}$ & $8,9,5,7$ \\
\hline
\end{tabular}


For $p=7$, we have $G=E_{7}, E_{8}$ and either the Sylow $p$-subgroup is abelian, hence we are done by above, or $p \mid(q \pm 1)$, whence the order of $u$ is at most $7(q \pm 1)(q-1) \leq 7\left(q^{2}-1\right)$, where the Weyl contribution is 7 , the toral contribution is $(q \pm 1)$, and the outer contribution is at most $(q-1)$. The product of these is clearly less than $q^{8}-1$.

For $p=5$, we have that the order of $u$ is at most $5\left(q^{2}+1\right)(q-1) \leq 5\left(q^{3}-1\right)$, using the same argument, as $d=1,2,4$. Of course, this is still smaller than $q^{7}-1$, which is the required bound as $G=E_{6}, E_{7}, E_{8}$.

For $p=3$, the order of $u$ depends on which group we are in. The toral contribution is at most $q+1$, the Weyl contribution is 3 for $G_{2}, F_{4}$ and 9 otherwise, and the outer contribution is at most $3(q-1)$ (diagonal for ${ }^{\varepsilon} E_{6}$ and field automorphisms). For $G=G_{2}, F_{4}$ we get $3\left(q^{2}-1\right)$, which is at most $\left(q^{3}-1\right)$ for $G_{2}(q)$ (as $q \neq 2,3$ ), and at most $q^{5}-1$ for all $q \geq 2$, so we get at most $q^{\ell+1}-1$. For $G=E_{6}, E_{7}, E_{8}$, we get $27\left(q^{2}-1\right)$, which is less than $\left(q^{7}-1\right)$ for all $q \geq 2$, so in all cases again $q^{\ell+1}-1$ will do.

For $p=2$, we get that the toral contribution is at most $q+1$, the Weyl contribution is 2 for $G_{2}$ and 8 for all other groups, and the outer contribution is at most $(q-1)$ for $G_{2}, F_{4}$ and $E_{8}, 2(q+1)$ for ${ }^{\varepsilon} E_{6}$, and $(q-1)$ for $E_{7}$, by [7, Theorem 2.5.12], yielding at most $2\left(q^{2}-1\right)$ for $G_{2}$, which is less than $q^{3}-1$ for $q \geq 3$.

For $F_{4}$ we get $o(u) \leq 8(q+1) \leq\left(q^{5}-1\right)$, for ${ }^{\varepsilon} E_{6}$ we have $16\left(q^{2}-1\right)(q+1) \leq$ $\left(q^{7}-1\right)$ for all $q \geq 3$, and for $E_{7}, E_{8}$ we have $o(u) \leq 16(q+1)(q-1) \leq\left(q^{8}-1\right)$, as needed.

Now we know that every $p$-element in $G$ has order at most $q^{\ell+1}-1$, then we get that $\operatorname{dim}(M)<\alpha(G) \cdot\left(q^{\ell+1}-1\right)$, and we can apply the Landazuri-SeitzZalesskii bounds from [21] and [27].

\begin{tabular}{llrl}
\hline Group & Landazuri-Seitz & LS evaluated $\alpha(G)$ & $\begin{array}{l}\alpha(G) \cdot\left(q^{\ell+1}-1\right) \\
\text { evaluated }\end{array}$ \\
\hline$F_{4}(q \geq 4$ even $)$ & $\frac{q^{7}}{2}\left(q^{3}-1\right)(q-1)$ & $448(q=4) \leq 8$ & $\leq 248(q=4)$ \\
$F_{4}(q$ odd $)$ & $q^{6}\left(q^{2}-1\right)$ & $5832(q=3) \leq 7 \leq 1694(q=3)$ \\
${ }^{\varepsilon} E_{6}$ & $q^{9}\left(q^{2}-1\right)$ & $1536(q=2) \leq 9 \leq 1143(q=2)$ \\
$E_{7}$ & $q^{15}\left(q^{2}-1\right)$ & $98304(q=2) \leq 10 \leq 2550(q=2)$ \\
$E_{8}$ & $q^{27}\left(q^{2}-1\right)$ & $402653184(q=2) \leq 11 \leq 5621(q=2)$ \\
\hline
\end{tabular}

This proves that these groups have no non-trivial minimally active modules, but slightly better bounds are needed for the other groups, as the minimal faithful degrees are closer to $q^{\ell}$. 
Proposition 38. Let $G_{0}$ be the simple group $G_{2}\left(q^{t}\right)$ for $q^{t} \geq 5$, and let $u$ be a p-element of $G$ such that $G=\left\langle G_{0}, u\right\rangle$. There are no non-trivial, minimally active modules for $G$.

Proof. Let $G_{0}$ be a central extension of $G_{2}\left(q^{t}\right)$ for some prime power $q$ and some $t \geq 1$, and let $G$ be obtained by adding on a field automorphism of order $t$ to $G_{0}$. Since $G_{2}(2)^{\prime}=\operatorname{PSU}_{3}(3)$ we have already dealt with it, and we dealt with $G_{2}(3)$ and $G_{2}(4)$ in Proposition 36, we may assume that $q^{t} \geq 5$.

First suppose that $p \geq 5$, so that $t=1$ or $t \geq 5$. From [10] we have $\alpha(G) \leq 5$, and the Landazuri-Seitz bound [21] for $G$ is $q^{t}\left(q^{2 t}-1\right)$. As $p \geq 5$, the order of $u$ is at most one of $t \Phi_{d}(q)$ for $d=1,2,3,6$, with $d=3$ maximizing this, so we get

$$
5 t^{2}\left(q^{2}+q+1\right)>\alpha(G) \cdot(o(u)-1) \geq \operatorname{dim}(M) \geq q^{t}\left(q^{2 t}-1\right),
$$

with $t=1$ and $q=5$ as solutions, and no solutions for $t \geq 5$. For $G=G_{0}=$ $G_{2}(5)$, the primes other than 2,3,5 dividing $|G|$ are 7 and 31, each dividing it exactly once, whence we need a simple module of dimension at most $4 \cdot(31-1)=$ 120 , but 124 is the minimal degree.

Suppose that $p=3$, or $p=2$ and $q$ is not a power of 3. The toral contribution is at most $t(q+1)$, the Weyl contribution is at most 3 , and the outer contribution is $t$. Since $\alpha(u) \leq 5$, we get

$$
15 t^{2}(q+1)>\alpha(G) \cdot(o(u)-1) \geq \operatorname{dim}(M) \geq q^{t}\left(q^{2 t}-1\right),
$$

which has no solutions for $q^{t} \geq 5$.

If $p=2$ and $q$ is a power of 3 , then we get the toral contribution to be $t(q+1)$, the Weyl contribution to be 2 , and the outer contribution to be $2 t$, so similar to the above expression, and we get

$$
20 t^{2}(q+1)>q^{t}\left(q^{2 t}-1\right),
$$

which has no solutions for $q^{t} \geq 9$. This completes the proof.

Proposition 39. Let $G_{0}$ be the simple group ${ }^{3} D_{4}\left(q^{t}\right)$ for some $q$ and $t$, and let $u$ be a p-element of $G$ such that $G=\left\langle G_{0}, u\right\rangle$. There are no non-trivial, minimally active modules for $G$.

Proof. Let $G_{0}={ }^{3} D_{4}\left(q^{t}\right)$ for some prime power $q$ and some $t \geq 1$ (there are no central extensions), and let $G$ be obtained by adding on a field automorphism of order dividing $3 t$ to $G_{0}$ (see [7, Theorem 2.5.12]). Note that $\alpha(G) \leq 7$ by [10, Proposition 5.7].

If $p \geq 5$, then the Sylow $p$-subgroup of $G_{0}$ is abelian, so let $d$ be the order of $q^{t}$ modulo $p$, so that $d=1,2,3,6,12$. If $p \mid \Phi_{12}\left(q^{t}\right)$, then from the list of maximal subgroups in [18], we see that $C_{G_{0}}(u)$ is abelian, so $\operatorname{dim}(M) \leq 2 o(u)$ if $M$ is min- 
imally active, by Lemma 8 . Furthermore, $o(u) \leq t^{2} \Phi_{12}(q)=t^{2}\left(q^{4}-q^{2}+1\right)$, and $\operatorname{since} \operatorname{dim}(M) \geq q^{3 t}\left(q^{2 t}-1\right)$ from [21], we get

$$
2 t^{2}\left(q^{4}-q^{2}-1\right) \geq \operatorname{dim}(M) \geq q^{3 t}\left(q^{2 t}-1\right),
$$

which has only the solution $q^{t}=2$, where $p=13$. Here $\operatorname{dim}(M) \geq 26=2 o(u)$ by [16, p. 253] so there are no non-trivial minimally active modules here.

If $d=1,2,3,6$, then the toral contribution is at most $t\left(q^{2}+q+1\right)$, and the outer contribution is $t$; since $\alpha(u) \leq 7$, we get that if $M$ is minimally active, then

$$
7 t^{2}\left(q^{2}+q+1\right) \geq q^{3 t}\left(q^{2 t}-1\right),
$$

where $q^{t}=2$ is again the only solution, this time with $p=7$. A quick computer check shows that for $p=7$ we actually have $\alpha(u)=2$, so that there are no nontrivial minimally active modules here either.

Thus $p=2,3$ remain. If $p=3$, then the order of $u$ is at most $9 t^{2}(q+1)$ by Proposition 26, using the fact that the exponent of the Weyl group of type $D_{4}$ is 12 . Since $\alpha(u) \leq 7$, we have that

$$
7 \cdot 27 t^{2}(q+1) \geq \operatorname{dim}(M) \geq q^{3 t}\left(q^{2 t}-1\right),
$$

which is satisfied only for $t=1, q=2,3$, with $q=3$ not allowed as $p \nmid q$. If $p=2$, then $u$ has order at most $4 t^{2}(q+1)$, and again $\alpha(u) \leq 7$ so that

$$
7 \cdot 4 t^{2}(q+1) \geq \operatorname{dim}(M) \geq q^{3 t}\left(q^{2 t}-1\right),
$$

which only has a solution for $t=1$ and $q=2$, not of interest as $p=2$. Thus we need to consider $p=3, G_{0}={ }^{3} D_{4}(2)$.

It is easy to check by computer that for any 3 -element in $G_{0}$, two conjugates of it generate $G_{0}$, and $o(u) \leq 9$, so if $u \in G_{0}$, then $\operatorname{dim}(M) \leq 2 \cdot 8=16$, smaller than the minimal dimension of 25 [16, p. 251]. If $G / G_{0}$ has order 3, then the Sylow 3-subgroup still has exponent 9 , so either $o(u)=9$, in which case two conjugates of $u$ generate $G$ and $\operatorname{dim}(M) \leq 16$, or $o(u)=3$ and $\langle u\rangle$ lies outside $G_{0}$, and then $\alpha(G)(o(u)-1) \leq 14$, less than 25 . This completes the proof.

Proposition 40. Let $G_{0}$ be a central extension of a Ree or Suzuki group other than ${ }^{2} B_{2}(8)$. There are no non-trivial, minimally active modules for smash $G$.

Proof. Let $G_{0}$ be a central extension of a Suzuki group ${ }^{2} B_{2}\left(2^{2 n+1}\right)$ for some $n \geq 2$, so that $p$ is odd. The minimal faithful degree for $G_{0}$ is $2^{n}\left(2^{2 n+1}-1\right)$ from [21], and from [10, Proposition 5.8] we have that $\alpha(G) \leq 3$. Note also that there is no Weyl contribution as $p$ is odd: the toral contribution is a divisor of one of $2^{2 n+1}-1,2^{2 n+1}+2^{n+1}+1$ and $2^{2 n+1}-2^{n+1}+1$, hence at most $2^{2 n+1}+2^{n+1}+1$, and the outer contribution is $t \mid(2 n+1)$. 
This yields

$$
\begin{aligned}
3 t\left(2^{2 n+1}+2^{n+1}+1\right) & >\alpha(u) \cdot(o(u)-1) \\
& \geq \operatorname{dim}(M) \\
& \geq 2^{n}\left(2^{2 n+1}-1\right),
\end{aligned}
$$

which has no solutions for $t=1$, and for $t=2 n+1$ only works for $n \leq 5$. If $t>1$, then $p \mid(2 n+1)$ : if $p \mid\left(2^{2 n+1} \pm 2^{n+1}+1\right)$ and $p \mid(2 n+1)$, then $p \mid(2 \pm 2+1)$, so $p=5$. The other alternative is that $p \mid\left(2^{2 n+1}-1\right)$, in which case $p=1$, which is not allowed. Thus $p=5$ always, so we need to consider $n=2$ only, as this is the only case where 5 divides $2 n+1$.

Here we just need to be more precise, noting that the Sylow 5-subgroup of ${ }^{2} B_{2}(32) .5$ has order 125 but exponent 25 , so actually $\operatorname{dim}(M) \leq 72$, less than the minimal degree of 124 .

We perform a similar analysis for the Ree groups $G_{0}={ }^{2} G_{2}\left(3^{2 n+1}\right)$ for $n \geq 1$, where the Landazuri-Seitz bound is $3^{2 n+1}\left(3^{2 n+1}-1\right)$, and from [10, Proposition 5.8] we have that $\alpha(G) \leq 3$. If $p=2$, then $o(u)=2$ by Proposition 26, so $\operatorname{dim}(M) \leq 3$ if $M$ is minimally active, absurd; thus $p$ is odd.

The order of any semisimple element of $G_{0}$ is a divisor of one of $3^{2 n+1}-1$, $3^{2 n+1}+1,3^{2 n+1}+3^{n+1}+1$ and $3^{2 n+1}-3^{n+1}+1$, and the outer contribution is at most $2 n+1$ : whence for $u \in G$,

$$
\begin{aligned}
3 \cdot(2 n+1) \cdot\left(3^{2 n+1}+3^{n+1}+1\right) & \geq 3 \cdot(o(u)-1) \\
& \geq \operatorname{dim}(M) \\
& \geq 3^{2 n+1}\left(3^{2 n+1}-1\right),
\end{aligned}
$$

if $u$ acts minimally actively, which fails for all $n \geq 1$.

We end with the group $G_{0}={ }^{2} F_{4}\left(2^{2 n+1}\right)$. Here the Landazuri-Seitz bound is $2^{9 n+4}\left(2^{2 n+1}-1\right)$, and $\alpha(G) \leq 7$. The toral contribution is at most one of $2^{2 n+1} \pm 1,2^{2 n+1} \pm 2^{n+1}+1,2^{4 n+2} \pm 2^{3 n+2}+2^{2 n+1} \pm 2^{n+1}+1$, the Weyl contribution is 3 , and the outer contribution divides $2 n+1$. Thus from the formula $\operatorname{dim}(M) \leq \alpha(G)(o(u)-1)$ for minimally active $M$, we get

$$
\begin{aligned}
21(2 n+1)\left(2^{4 n+2}+2^{3 n+2}+2^{2 n+1}+2^{n+1}+1\right) & >\alpha(G)(o(u)-1) \\
& \geq \operatorname{dim}(M) \\
& \geq 2^{9 n+4}\left(2^{2 n+1}-1\right) .
\end{aligned}
$$

The only solution to this is $n=0$, i.e., $G_{0}$ is the Tits group. Here it is easy to check that $G_{0}$ is generated by two conjugates of any element of order at least 3, that $u$ has order at most 13 , and that $\operatorname{dim}(M) \geq 26$, thus there is no example here. 


\section{Proof of Theorem 2}

In this section we need to check that all of the minimally active modules that we have found satisfy Theorem 2 .

Proposition 41. If $G_{0} / Z\left(G_{0}\right)$ is an alternating group, then Theorem 2 is satisfied.

Proof. We check the twenty-nine examples from Proposition 16 first, noting that the symmetric group in its natural module is a complex reflection group; this checks (i)-(iii). Cases (iv) and (v) are $G_{0}=\mathrm{SL}_{2}(4)$, so these are fine. Most of the other cases are where $G / Z(G)$ has a self-centralizing cyclic Sylow $p$-subgroup, where $\operatorname{dim}(M) \leq p+1$ : all of the cases where $p=5,7$ have this property, so we are left with (x)-(xiv), (xviii)-(xix) and (xxvi)-(xxviii), with these last three cases being the natural module for $\mathrm{SL}_{4}(2)$, so covered.

For (x)-(xiv) we have $G_{0} / Z\left(G_{0}\right)$ being Alt $_{6}$. Case (xii) is the complex reflection group $G_{27}$, and cases (xiii) and (xiv) are $\mathrm{SL}_{2}(9)$, leaving (x) and (xi). In (x) the module is imprimitive, as is the 6-dimensional module in (xi), so we are left with the 9-dimensional module for $3 \cdot M_{10}$, which is on our list of exceptions.

Finally, case (xviii) is the restriction of the same module for Alt 8 , and case (xix) is again on our list of exceptions.

The remaining alternating groups are from Proposition 18, where we either have the permutation module or $G=2$. Alt9 and $\operatorname{dim}(M)=8$, and this is the restriction of the reflection representation of the Weyl group of $E_{8}, G_{37}$.

Proposition 42. If $G_{0} / Z\left(G_{0}\right)$ is a sporadic group, then Theorem 2 is satisfied.

Proof. These are given in Proposition 23. We first remove those cases where there is a self-centralizing, cyclic Sylow $p$-subgroup, which are (i)-(iii), (v)-(vii), (ix)-(xiv), (xvi) and (xvii). The remaining three cases are all for $p=2$, and are on our list of exceptions.

Proposition 43. If $G_{0} / Z\left(G_{0}\right)$ is a group of Lie type in cross characteristic, then Theorem 2 is satisfied.

Proof. We start with the groups and modules in Proposition 24. Cases (vi)-(vii) are $G_{34}$, cases (ix) and (x) are the Weyl group of $E_{7}, G_{36}$, and (xii)-(xiv) are the Weyl group of type $E_{8}, G_{37}$.

Cases (i)-(v) have cyclic Sylow $p$-subgroups that are self centralizing, while (ii) is also contained in $G_{34}$ and (iv) with the 6-dimensional module if $G_{35}$. The remaining case is (xi), which is contained in the Weyl group of type $E_{8}$.

The companion proposition to Proposition 24 for exceptional groups is Proposition 36, and all the groups in this have self-centralizing, cyclic Sylow 13-sub- 
groups, and the group $\mathrm{Sp}_{4}(4)$ has a self-centralizing, cyclic Sylow 17-subgroup, as in Proposition 32. The remaining non-Weil module is from Proposition 30, and $\mathrm{PSL}_{3}(3)$ has a self-centralizing Sylow 13-subgroup of order 13.

If $G_{0}$ is a central extension of $\operatorname{PSL}_{2}(q)$, then in all cases the Sylow $p$-subgroup is cyclic and self centralizing, so this case is covered.

If $M$ is a Weil module then $u$ is a Singer cycle and so the Sylow $p$-subgroup is cyclic and self-centralizing, as is the Sylow 7-subgroup of $\mathrm{SU}_{3}(3)$, so these are also covered by the theorem.

Proposition 44. If $G_{0} / Z\left(G_{0}\right)$ is a group of Lie type in defining characteristic, then Theorem 2 is satisfied.

Proof. Each of the modules listed in Proposition 19 appears on our list, so $u$ induces an outer automorphism, and appears in Propositions 20 and 21.

For the groups in Proposition 20, (i) and (iv) are imprimitive, (v) is the natural module, (iii) is the exterior square of the natural, and (ii) is mentioned explicitly. The groups in Proposition 21 are either imprimitive in case (v), or stabilize a tensor product in cases (i)-(iv).

Acknowledgments. The author would like to thank Donna Testerman and Alex Zalesski for giving him access to unpublished manuscripts of theirs.

\section{Bibliography}

[1] M. Aschbacher, On the maximal subgroups of the finite classical groups, Invent. Math. 76 (1984), no. 3, 469-514.

[2] J. H. Conway, R. T. Curtis, S. P. Norton, R. A. Parker and R. A. Wilson, Atlas of Finite Groups. Maximal Subgroups and Ordinary Characters for Simple Groups, Clarendon Press, Oxford, 1985.

[3] D. A. Craven, B. Oliver and J. Semeraro, Reduced fusion systems over $p$-groups with abelian subgroup of index $p$ : II, Adv. Math. 322 (2017), 201-268.

[4] L. Di Martino, M. A. Pellegrini and A. E. Zalesski, On generators and representations of the sporadic simple groups, Comm. Algebra 42 (2014), no. 2, 880-908.

[5] L. Di Martino and A. E. Zalesski, Almost cyclic elements in Weil representations of finite classical groups, Comm. Algebra 46 (2018), no. 7, 2767-2810.

[6] J.D. Dixon and B. Mortimer, Permutation Groups, Grad. Texts in Math. 163, Springer, New York, 1996.

[7] D. Gorenstein, R. Lyons and R. Solomon, The Classification of the Finite Simple Groups. Number 3. Part I. Chapter A, Math. Surveys Monogr. 40, American Mathematical Society, Providence, 1998. 
[8] R. Gow and M. C. Tamburini, Generation of $\operatorname{SL}(n, \mathbf{Z})$ by a Jordan unipotent matrix and its transpose, Linear Algebra Appl. 181 (1993), 63-71.

[9] R. M. Guralnick, K. Magaard, J. Saxl and P. H. Tiep, Cross characteristic representations of symplectic and unitary groups, J. Algebra 257 (2002), no. 2, 291-347.

[10] R. M. Guralnick and J. Saxl, Generation of finite almost simple groups by conjugates, J. Algebra 268 (2003), no. 2, 519-571.

[11] R. M. Guralnick and P.H. Tiep, Low-dimensional representations of special linear groups in cross characteristics, Proc. Lond. Math. Soc. (3) 78 (1999), no. 1, $116-138$.

[12] G. Hiss and G. Malle, Low-dimensional representations of special unitary groups, J. Algebra 236 (2001), no. 2, 745-767.

[13] C. Hoffman, Cross characteristic projective representations for some classical groups, J. Algebra 229 (2000), no. 2, 666-677.

[14] G. D. James, On the minimal dimensions of irreducible representations of symmetric groups, Math. Proc. Cambridge Philos. Soc. 94 (1983), no. 3, 417-424.

[15] C. Jansen, The minimal degrees of faithful representations of the sporadic simple groups and their covering groups, LMS J. Comput. Math. 8 (2005), 122-144.

[16] C. Jansen, K. Lux, R. Parker and R. Wilson, An Atlas of Brauer Characters, London Math. Soc. Monogr. (N. S.) 11, Clarendon Press, New York, 1995.

[17] W. M. Kantor, Subgroups of classical groups generated by long root elements, Trans. Amer. Math. Soc. 248 (1979), no. 2, 347-379.

[18] P. B. Kleidman, The maximal subgroups of the Steinberg triality groups ${ }^{3} D_{4}(q)$ and of their automorphism groups, J. Algebra 115 (1988), no. 1, 182-199.

[19] P. Kleidman and M. Liebeck, The Subgroup Structure of the Finite Classical Groups, London Math. Soc. Lecture Note Ser. 129, Cambridge University Press, Cambridge, 1990.

[20] A.S. Kleshchev and P. H. Tiep, Small-dimensional projective representations of symmetric and alternating groups, Algebra Number Theory 6 (2012), no. 8, 1773-1816.

[21] V. Landazuri and G. M. Seitz, On the minimal degrees of projective representations of the finite Chevalley groups, J. Algebra 32 (1974), 418-443.

[22] R. Lawther, Jordan block sizes of unipotent elements in exceptional algebraic groups, Comm. Algebra 23 (1995), no. 11, 4125-4156.

[23] M. W. Liebeck and G. M. Seitz, Unipotent and Nilpotent Classes in Simple Algebraic Groups and Lie Algebras, Math. Surveys Monogr. 180, American Mathematical Society, Providence, 2012.

[24] F. Lübeck, Small degree representations of finite Chevalley groups in defining characteristic, LMS J. Comput. Math. 4 (2001), 135-169. 
[25] G. Malle and D. Testerman, Linear Algebraic Groups and Finite Groups of Lie Type, Cambridge Stud. Adv. Math. 133, Cambridge University Press, Cambridge, 2011.

[26] G. M. Seitz, The maximal subgroups of classical algebraic groups, Mem. Amer. Math. Soc. 365 (1987), 1-286.

[27] G. M. Seitz and A. E. Zalesskii, On the minimal degrees of projective representations of the finite Chevalley groups. II, J. Algebra 158 (1993), no. 1, 233-243.

[28] T. A. Springer, Regular elements of finite reflection groups, Invent. Math. 25 (1974), 159-198.

[29] I.D. Suprunenko, Unipotent elements of nonprime order in representations of the classical algebraic groups: Two big Jordan blocks, J. Math. Sci. 199 (2014), 350-374.

[30] D. M. Testerman and A. E. Zalesski, Irreducible representations of simple algebraic groups in which a unipotent element is represented by a matrix with a single nontrivial Jordan block, J. Group Theory 21 (2018), no. 1, 1-20.

[31] R. A. Wilson, The Finite Simple Groups, Grad. Texts in Math. 251, Springer, London, 2009.

[32] T.E. Zieschang, Primitive permutation groups containing a p-cycle, Arch. Math. (Basel) 64 (1995), no. 6, 471-474.

Received June 27, 2017; revised April 12, 2018.

\section{Author information}

David A. Craven, School of Mathematics, University of Birmingham, Edgbaston, Birmingham, B15 2TT, United Kingdom.

E-mail: d.a.craven@bham.ac.uk 